ANNALES

UNIVERSITATIS MARIAE CURIE-SKŁODOWSKA

LUBLIN - POLONIA

VOL. LXIX, 1-2

SECTIO AA

2014

\title{
Positronium as a probe of small free volumes in crystals, polymers and porous media
}

\author{
Tomasz Goworek \\ 20-031 Lublin, Poland, \\ tomasz.goworek@poczta.umcs.lublin.pl
}

\begin{abstract}
Positronium (a hydrogen-like bound state of an electron and a positron) is a convenient probe to determine the sizes of subnanometric free volumes (voids) in condensed matter. A review of experimental methods used in positron spectroscopy and examples of their application to the free volume studies are presented.
\end{abstract}

\section{INTRODUCTION}

Positronium (Ps) is a bound structure of an electron and positron, thus it is a kind of hydrogen-like atom with reduced mass equal to half of electron mass, $m^{\prime}=m_{e} / 2$. As a consequence, the binding energy of its ground state is half of that for hydrogen $-6.8 \mathrm{eV}$, the most probable distance of particles is $2 a_{0}$ (where $a_{0}=0.053 \mathrm{~nm}$ is the Bohr radius). The positronium was discovered in 1951 by Deutsch [1] in the processes of positron slowing down in gases.

Energetic positrons entering matter lose their energy by ionization, excitation of the medium molecules, production of radicals etc. The cross section for ionization is of the order of $10^{-16} \mathrm{~cm}^{2}$, while the cross section for the simplest, two-quantum, annihilation vary from $10^{-22} \mathrm{~cm}^{2}$ for positrons with the energy comparable to that of atomic valence electrons, to $5 \cdot 10^{-25} \mathrm{~cm}^{2}$ for positrons with the energy $100 \mathrm{keV}$. Thus, the annihilation ,,in flight” is negligible and the transformation of electron- 
positron pair into gamma quanta occurs almost exclusively after positron thermalization. At this stage the direct annihilation competes with that preceded by the formation of the bound state.

The states of Ps show a hyperfine structure. Depending on mutual spin orientation of involved particles we have the singlet state (antiparallel spins) called para-positronium, $p$-Ps, or triplet state (parallel spins) - ortho-positronium, $o$-Ps. Due to the parity conservation law $p$-Ps can annihilate from its ground state with the emission of an even number of $\gamma$-quanta, $o$-Ps - of an odd number (one-quantum annihilation of free o-Ps is forbidden). The lifetime of $p$-Ps decaying in vacuum into two gamma quanta $511 \mathrm{keV}$ is $\tau_{S}=125 \mathrm{ps}$, the three-quantum process of o-Ps disappearance occurs relatively slowly, the mean lifetime of o-Ps in vacuum is $\tau_{T}=142 \mathrm{~ns}$; the energy spectrum of quanta is continuous $(0 \div 511) \mathrm{keV}$. Just that presence of long-lived component in the positron lifetime distribution is the hallmark of o-Ps formation and served for Ps identification in Deutsch's experiment. The processes with emission of four or more quanta can be neglected; the probability of decay decreases rapidly with the number of emitted quanta, e.g. the ratio of probabilities (branching ratio) of four- and two-quantum annihilation is about $1.5 \cdot 10^{-6}$ [2]. Quantum electrodynamics predicts also an exotic decay of Ps into a pair neutrino-antineutrino, but the branching ratio is $6 \cdot 10^{-18}$ [3].

Soon after the discovery of positronium in gases it was reported that similar bound structures can be formed also in molecular substances, liquid and solid $[4,5]$. Their properties (decay mode, lifetime, momentum at the time of annihilation), depending on the properties of medium in which they reside, open the possibility to use Ps as a tool in the study of condensed matter. In particular, one can use positronium techniques to determine the sizes of free (i.e. electron-less) volumes in solid, in the range from $0.2 \mathrm{~nm}$ to about $50 \mathrm{~nm}$.

In condensed matter Ps is formed almost exclusively in its ground state, due to the low binding energy and large size of that atom in excited states. Positronium in these states can be created as a result of interaction of slow positrons with surfaces, when newly formed Ps escapes into vacuum. Excitation of free Ps atoms is possible by resonance absorption of tunable laser light. All fundamental properties of Ps atom like the hyperfine structure of low-lying levels, respective lifetimes to annihilation and to the optical transitions between the levels, the behaviour in magnetic or electric fields were calculated and confirmed experimentally. Long lasting controversies between experimental results for the o-Ps 
lifetime in vacuum [6] and its predictions by quantum electrodynamics [7] were finally eliminated [8].

$$
* * *
$$

The structure and properties of the positronium excited states are not the subject of this paper, respective data one can find in the review articles [I-III].

\section{POSITRON AND POSITRONIUM SOURCES}

\subsection{Radioactive sources}

As positron sources the isotopes decaying via $\beta^{+}$process, e.g. ${ }^{22} \mathrm{Na}$, ${ }^{44} \mathrm{Ti}^{44} \mathrm{Sc},{ }^{68} \mathrm{Ga}$, are commonly used. The advantage of a ${ }^{22} \mathrm{Na}$ source (beside its long half-life, $2.7 \mathrm{y}$ ) is that the beta decay populates the excited state of a ${ }^{22} \mathrm{Ne}$ nucleus, which in very short time $(\approx 5 \mathrm{ps})$ de-excites emitting the $1274 \mathrm{keV}$ gamma ray. The appearance of that gamma quantum can serve as a signal of positron birth. Also the decay of ${ }^{44} \mathrm{Sc}$ is accompanied by the emission of a $1160 \mathrm{keV}$ quantum.

In the majority of experiments the positron source is in the direct contact with the investigated sample (in a sandwich between two samples). In order to avoid sample contamination by $\beta^{+}$activity and allow its multiple use, the radioactive source is usually sealed in a thin envelope. It can be made of metal foil (e.g. nickel $\approx 1 \mu \mathrm{m}$ thick), or polyimide Kapton foil, $(6 \div 10) \mu \mathrm{m}$ thick. Such foils absorb about $10 \%$ of positrons from the ${ }^{22} \mathrm{Na}$ source and respective correction should be introduced at processing the experimental data. The Kapton foil is the most convenient source envelope, as it introduces one lifetime component only. Moreover, this lifetime, $380 \mathrm{ps,} \mathrm{practically} \mathrm{does} \mathrm{not} \mathrm{depend} \mathrm{on}$ temperature. The ${ }^{22} \mathrm{Na}$ activity is usually deposited in the form of $\mathrm{NaCl}$ solution and then dried. $\mathrm{NaCl}$ corrodes some metal backings if not protected by a very thin non-corroding layer, e.g. of gold (a few $\mathrm{nm}$ ). The ${ }^{22} \mathrm{Na}$ activity in the form of $\mathrm{Na}_{2} \mathrm{CO}_{3}$ is sometimes used.

As an example of positron source without envelope one can mention the glass foil with Na diffused in at high temperature [9]; the sources with ${ }^{22} \mathrm{Na}$ implanted as high energy ions into metallic foils [10,11] did not find practical application yet. In the study of liquids the ${ }^{22} \mathrm{Na}$ activity can be dissolved directly in the sample volume. 


\subsection{Pair creation}

Another source of positrons is via the creation of electron-positron pairs in the interaction of high energy quanta with matter. Hitting the target they transform into electron-positron pairs separated then by the magnetic field. Such quanta can be produced in two ways ${ }^{1}$ :

- as the Bremsstrahlung of high energy electrons accelerated in a synchrotron,

- at the inverse Compton scattering, i.e. at head-on collisions of laser quanta with high energy electrons.

An interesting variant of that technique is the creation of pairs in situ, inside the investigated sample. This is important, when, on certain reasons, like high sample temperature, it is not possible to place the radioactive source in contact with the sample. For example, in the experiment by Hirade et al. [12] X-rays of 10-20 MeV, produced by the inverse Compton effect, transformed into $\mathrm{e}^{+} \mathrm{e}^{-}$pairs when entered the investigated sample ( $\mathrm{Ta}$ or Ge in this experiment).

\subsection{Slow positron beams}

In both processes, i.e. beta decay and pair production, the energy spectrum of positrons is continuous and extends over a broad range. In the case of ${ }^{22} \mathrm{Na}$ that range is $(0-550) \mathrm{keV}$, in ${ }^{68} \mathrm{Ge}(0-1.9) \mathrm{MeV}$. In certain experiments one needs to have a monoenergetic beam of positrons. It can be produced due to the negative work function for positrons in some solids. The positrons from a conventional source enter the solid, thermalise, and some of them are re-emitted if they approach the surface in their diffusional motion. The energies of re-emitted positrons correspond to their work function, i.e. (1-3) eV $[13,14,15]$. Then, they can be accelerated to the needed energy by application of a voltage between emitter and accelerating electrode. The efficiency of slow positron emission is very low, $10^{-3}-10^{-4}$ per one positron implanted into the medium. The most popular medium with negative positron work function is tungsten single crystal in the form of foil with the surface in (110) crystalline direction. The efficiency in this case can reach to $3 \cdot 10^{-4}$, the energy of re-emitted positrons is $3 \mathrm{eV}$. The highest efficiencies are obtained using solidified noble gases, e.g. neon [16], however, this technique is not too convenient to use.

\footnotetext{
${ }^{1}$ Historically, the first high energy radiation used to produce $\mathrm{e}^{+} \mathrm{e}^{-}$pairs were gamma quanta 2.6 MeV from natural radioisotope ThC'.
} 
Typical arrangement of the slow positron beam is shown in Fig. 1. Positrons from a ${ }^{22} \mathrm{Na}$ source hit the $\mathrm{W}$ foil $(1 \div 2) \mu \mathrm{m}$ thick. At this thickness about $90 \%$ of fast positrons pass through, together with reemitted ones. They are accelerated by a small voltage and enter a weak magnetic field $(5 \div 10) \mathrm{mT}$, produced by a long coil. This field is too weak to change considerably the direction of primary beta rays, but the slow positrons are deflected and guided in a helical motion along the coil axis. The beam of slow positron can be then accelerated (or decelerated) to a selected energy.

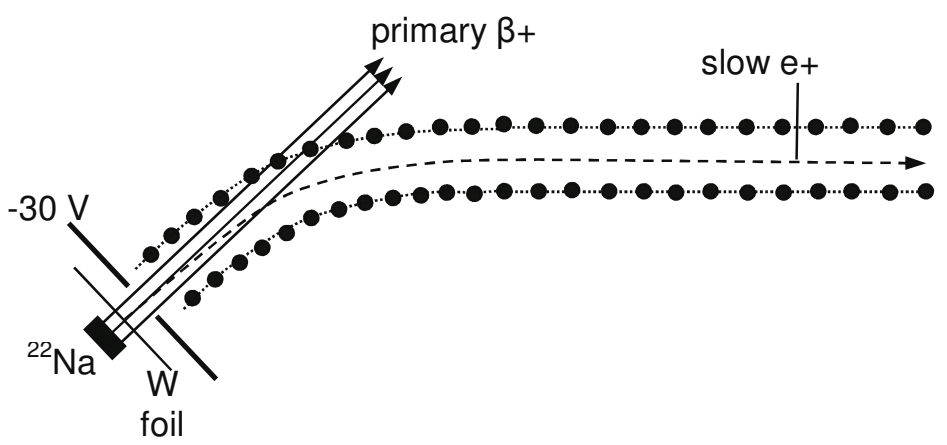

Fig.1. Slow positron source.

It is also possible to form the short bunches of monoenergetic positrons, i.e. pulsed beams of particles. This technique will be described in Sec.3.1.2.

\subsection{Positronium beams}

Usually, in application to the investigations of condensed media, positronium is formed inside the medium under study, however, if one wants to observe the interaction of Ps with surfaces or molecules of gas, it becomes necessary to have free Ps in vacuum. It can be produced with high efficiency when the slow positron beam hits the surface of solid; it can pick up an electron and be re-emitted as Ps with particularly low energy. When the target is heated to the temperatures close to its melting point, the efficiency of Ps formation by positrons hitting the target with energies below $100 \mathrm{eV}$ can approach 97\% [17]. Particularly effective is the target made of germanium with the surface in (100) direction.

In some experiments, like Ps Bose-Einstein condensation, gravitation study of antihydrogen, Rydberg states of Ps, one needs to produce thermalized positronium (in particular at very low temperatures). It can be done by implanting $\mathrm{e}^{+}$beam with the energy of several $\mathrm{keV}$ into ordered porous silica (see Sec.11.2). Ps formed in silica and emitted into the open 
pores thermalizes and diffuses to outside [18]. The efficiency of $\mathrm{e}^{+}-\mathrm{Ps}$ transformation exceeds $50 \%$.

The positronium beam of tunable energy can be produced employing the photodetachment of $\mathrm{Ps}^{-}$, i.e. the bound state of positron and two electrons [19]. Ionizing potential of this negative Ps ion is $0.33 \mathrm{eV}$. In the setup described by Michishio et al. [20] the monoenergetic beam of positrons bombards the target of tungsten foil covered by a monolayer of $\mathrm{Na}$. The Ps- ions emitted from this surface (efficiency about 1.5\%) are accelerated by a static electric field. The $\mathrm{Ps}^{-}$beam is then illuminated by $1074 \mathrm{~nm}$ laser detaching the electron from about $50 \%$ of ions.

\section{METHODS OF POSITRONIUM OBSERVATION}

Fig. 2 shows the spectrum of pulse amplitudes produced by a germanium detector registering the annihilation radiation.

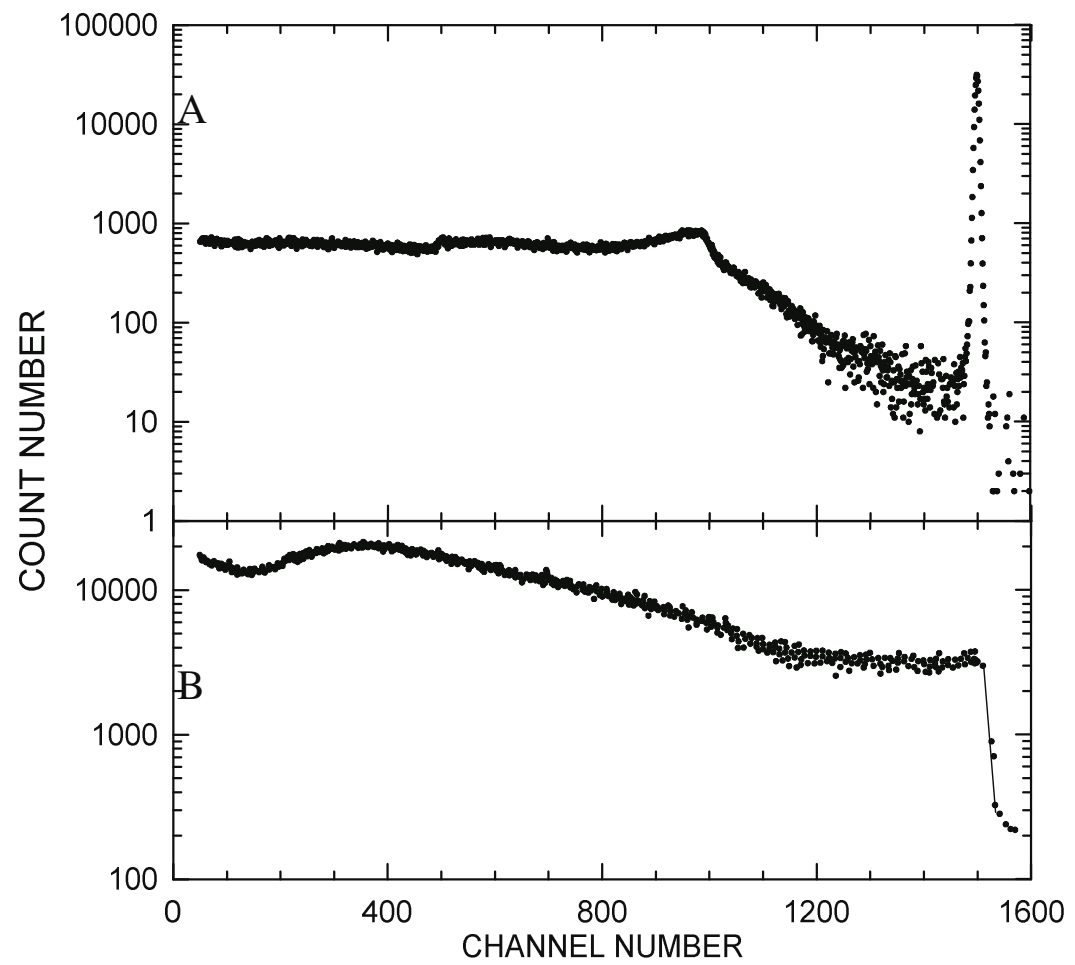

Fig. 2. Pulse amplitude spectrum of annihilation radiation registered by germanium detector. A - two-quantum annihilation (single $511 \mathrm{keV}$ line, broadened by Doppler effect), B - three-quantum annihilation (continuous energy spectrum $0-511 \mathrm{keV})$. Spectrum B is collected with very high statistics. 
The gamma quanta of energy $E_{\gamma}$ give the pulse amplitudes corresponding to the total energy and continuous distribution related to the energy left by recoil electrons at Compton scattering. The spectrum A is for two-quantum annihilation, B - for three-quantum one. The pulse amplitude spectra produced by detectors are superpositions of the spectra type $\mathrm{A}$ and $\mathrm{B}$; in the case of $\mathrm{a}^{22} \mathrm{Na}$ source the spectrum contains additionally the pulses belonging to $1274 \mathrm{keV}$ gamma rays.

\subsection{Positron annihilation lifetime spectroscopy (PALS)}

\subsubsection{Lifetime spectrometer}

The distribution of positron lifetimes is measured using the delayed coincidence spectrometer, usually of "fast-fast" type. As a rule the ${ }^{22} \mathrm{Na}$ source (about $10 \mu \mathrm{Ci}$ ) is used. The birth of positron is signalized by registration of the $1274 \mathrm{keV}$ gamma quantum from the ${ }^{22} \mathrm{Na}$ source in the scintillation detector, the stop signal denoting the death of positron comes from the detector registering one of two $511 \mathrm{keV}$ annihilation quanta. The simplified block scheme of the spectrometer is shown in Fig. 3. To avoid summing-up effects produced by scattered radiation the scintillation counters cannot be placed in face-to-face setting [21], but rather at an angle like $90^{\circ}$. The signals from the counters are sent to the differential constant fraction discriminators (DCFD). They choose from the spectrum of pulse amplitudes only those corresponding to the energies around the selected one. In the start counter it is the energy $1274 \mathrm{keV}$, in the stop counter it is usually the energy of two-quantum annihilation radiation $511 \mathrm{keV}$. When the three quantum annihilation dominates and produces the continuous gamma spectrum, that "energy window" should be broadened toward lower energies. Discriminators generate the timing signals to the time-to-amplitude converter (TAC). At the output of TAC one obtains the pulses with the amplitude proportional to time elapse between start and stop signals; these pulses are then recorded in respective channels of a multichannel amplitude analyser (MCA). Sometimes in the PALS spectrometers the pulse amplitude selection and timing process are separated (fast-slow spectrometer); they can contain also some extra circuitry like the units rejecting the events of pulse pile-up. 


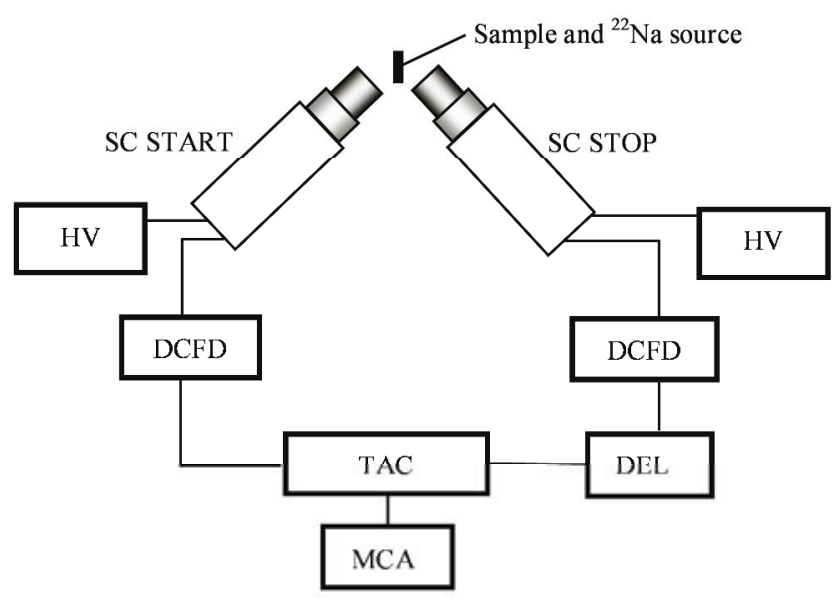

Fig. 3. Block scheme of the delayed coincidence spectrometer (in the simplest fast-fast version). HV - high voltage supply; SC - scintillation counter, DCFD - differential constant fraction discriminator, DEL - delay line, TAC - time to amplitude converter, MCA - multichannel analyser.

To obtain the best time resolution of the spectrometer, the scintillators should have the rise time of the signal as short as possible. During long time the fastest scintillators were plastic ones (Pilot U, NE 111). However, being built of low $Z$ elements, they practically did not contain the full energy peak in the spectrum of amplitudes; the signals belonging to respective quanta were selected from the fragments of Compton continua. Introduction of $\mathrm{BaF}_{2}$ crystals [22] producing intense full energy pulses and very fast rising light component in the UV region, allowed easier selection of quanta, better timing and ensured higher efficiency of counting.

Recently a new version of lifetime spectrometer appeared, in which DCFD discriminators are not used. The start and stop signals are analysed in a digital way. The front of the pulse from the scintillation counter is strobed with the frequency of several $\mathrm{GHz}$ and respective programs choose the appropriate moment for emission of timing signal. It allows to improve the resolution of spectrometer by about $20 \%$ [23].

\subsubsection{Generation of the start signal}

In the standard spectrometers the START signal comes from a gamma ray absorbed in the scintillator. Alternatively the START signal can be generated at the transition of positron through a thin plastic scintillator, before entering the sample [24]. Passing through the scintillator $0.5 \mathrm{~mm}$ 
thick, positron loses about $140 \mathrm{keV}$ of its energy; thus with a ${ }^{22} \mathrm{Na}$ source (mean $\beta^{+}$energy $\sim 180 \mathrm{keV}$ ) the fraction of transmitted positrons would be substantially reduced. The method is better suited to such sources like ${ }^{68} \mathrm{Ga}$, with mean $\beta^{+}$energy about $650 \mathrm{keV}$. The advantage of such START signal production is $100 \%$ efficiency of positron registration, that speeds up the data collection. In the setup with positron transmitting detector, one sample only is needed, while in traditional version, two pieces of sample have to be placed on both sides of the source.

Instead of a thin scintillator Shirai et al. $[25,26]$ propose to use a silicon avalanche diode. The signal produced when the positron penetrates the diode has the rise time of about $1.5 \mathrm{~ns}$, i.e similar to that from the fast scintillator, thus diode detector does not deteriorate the resolution of the spectrometer. A disadvantage can be small surface of the detector $(\approx 5 \times 5 \mathrm{~mm})$.

In the case of slow positron beams generation of the start signal needs other solutions. It can be realized in two ways:

a) monitoring the positron by secondary electrons,

b) applying the pulsed beams.

The method a) was developed by Lynn, Frieze and Schultz [27]. Slow positrons, accelerated to the energy of several $\mathrm{keV}$ are directed to a nickel foil remoderator; secondary electrons ejected from the foil are detected by a channel electron multiplier, forming the start pulse. The remoderated positrons are transported to the sample chamber (Fig. 4).

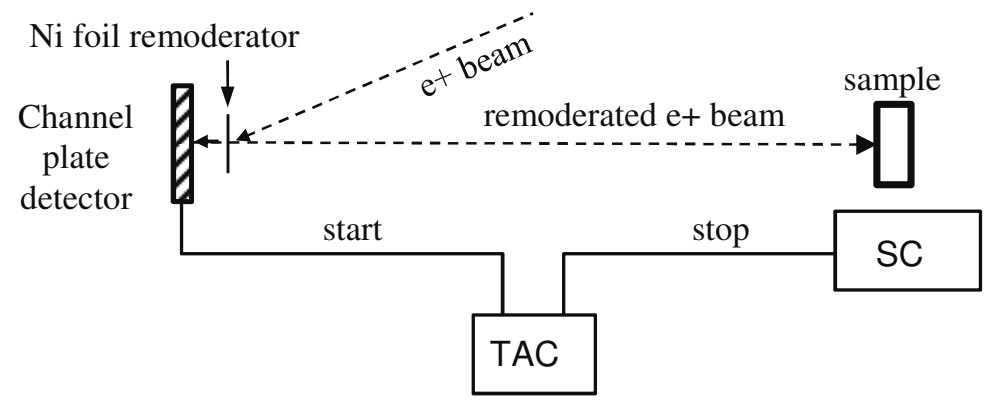

Fig. 4. Experimental setup for the lifetime measurements with positron.

In the method b) short pulses of positrons can be produced when their beam passes through a sequence of accelerating and decelerating fields. An example of such pulsating source, as designed in Aalto University [28], is shown below (Fig. 5). The beam of slow positrons 
enters the prebuncher fed by radio frequency (RF) sine wave voltage 33.3 MHz and its first harmonic. At the first part of oscillation period the positrons which entered earlier get less energy than those which arrived later, and thus in a drift tube $\approx 1 \mathrm{~m}$ long they catch up each other and become primarily bunched (focused in time). Then they are accelerated by $1 \mathrm{kV}$ and pass through the "chopper" supplied by RF $16.6 \mathrm{MHz}$. The phase of oscillation in the chopper is matched to get the arrival of the bunch when the voltage at that device is near zero (twice per each cycle). The chopper is followed by next oscillator working at fifth harmonics, $166 \mathrm{MHz}$, forming the final bunch. At the output the duration of positron bunch is less than $200 \mathrm{ps}$, the cycle of repetition of the bunches is the same as of the prebuncher, i.e. their spacing is $30 \mathrm{~ns}$. The start signal is produced by the RF generator.

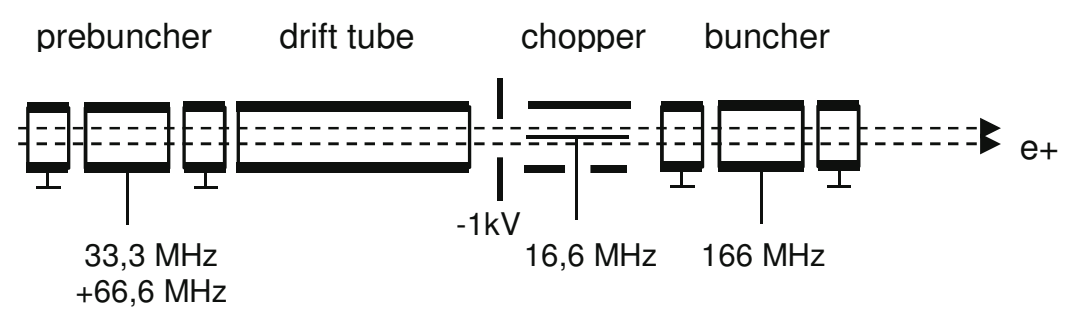

Fig. 5. Formation of the slow positron pulsed beam.

There is to note that repetition rate of the system is much higher than the flux of positrons $\left(\approx 10^{4} \cdot \mathrm{s}^{-1}\right)$. Thus, the term "bunch" cannot be understood literally, since the majority of cycles is empty and the remainder contains a single particle only. There is no production of the bunches of many particles, the role of buncher is to correlate precisely in time the arrival of particle at the output with the generated start pulse.

The situation described above appears at relatively weak flux of positrons, obtainable with a radioisotope as a primary source. When the positron beam is produced by the Bremsstrahlung from a powerful synchrotron accelerator, the positron flux at the output of installation, like ELBE in Dresden [29], can reach $7 \cdot 10^{8} \mathrm{~s}^{-1}$. In this case the real bunches of many positrons are produced (about 60 moderated positrons per bunch), the duration of pulse is reduced to $5 \mathrm{ps}$.

\subsubsection{Structure of spectrum}

The counting system has finite time resolution, i.e. for exactly simultaneous signals it produces the distribution of counts in time $P(x)$, 
where $x$ is the time shift observed experimentally. Thus, if the real distribution of lifetimes is $f(t)$, the instrumental distribution $N(x)$

$$
N(x)=\int_{0}^{\infty} P(x-t) f(t) d t+B
$$

where $x$ is the delay of stop signal after the start one (i.e. "the instrumental time"), $t$ - the real time, $B$ is the constant background produced by random coincidences. $P(x-t) d x$ means the probability that the event which occurred at the moment $t$ will be shifted by the spectrometer's electronics to the interval $\langle x, x+d x\rangle \quad\left(\int_{-\infty}^{+\infty} P(x) d x=1\right)$.The full width at half-maximum (FWHM) of the $P(x)$ curve is usually $(160 \div 300)$ ps, its shape can be approximated by the Gaussian (or a sum of Gaussians).

The positron in a definite state $i$ annihilates with the rate characteristic for that state $\lambda_{\mathrm{i}}$ (mean lifetime $\tau_{\mathrm{i}}=1 / \lambda_{\mathrm{i}}$ ). If in the sample positronium is formed, the distribution of annihilations in time contains at least 3 exponential components (Fig. 6) related to: annihilation of free positrons, para-Ps and ortho-Ps:

$$
f(t)=\left\{\begin{aligned}
\sum_{i=1}^{3} I_{i} \lambda_{i} \exp \left(-\lambda_{i} t\right), & t \geq 0 \\
0, & t<0
\end{aligned}\right.
$$

where $I_{i}$ is the relative intensity of $i$-th component $\left(\Sigma I_{i}=1\right)$.

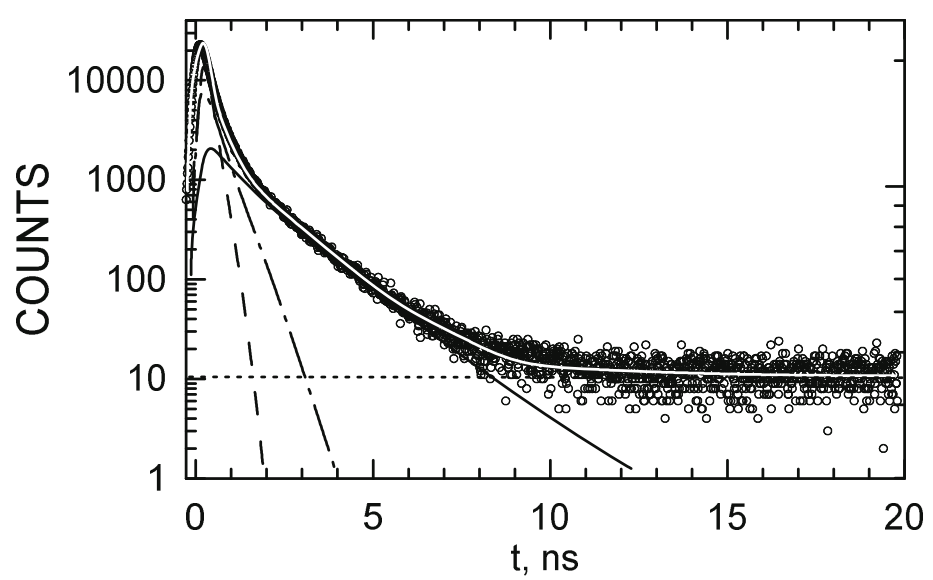

Fig. 6. The positron lifetime spectrum and its structure (exponentials related to particular annihilation processes, convoluted with instrumental resolution curve). Solid line - ortho-Ps, dashed - para-Ps, dash-dot - annihilation of free positrons, dots - random coincidence background. 
Due to statistical weights of para and ortho states the ratio of intensities of respective components should be 1:3 (provided that there are no additional processes of Ps transformation). The lifetime of para-Ps in vacuum is $\tau_{\mathrm{S}}=125 \mathrm{ps}$, and even if in the medium it is modified, it remains the shortest-lived component of the spectrum. Freely annihilating positrons (i.e. disappearing in collisions with electrons) in an organic medium produce the component with the lifetime $\tau_{2}=(250 \div 500) \mathrm{ps}$; the longest lifetimes observed in the PAL spectrum are ascribed to ortho-Ps. The ortho-Ps in vacuum has its intrinsic lifetime $\tau_{\mathrm{T}}=142 \mathrm{~ns}$, but while in the medium it has an additional possibility to annihilate not with its "own" electron, but with an electron from surrounding molecules, which has an opposite spin orientation. This process, called pick-off [30], is a two-quantum one and leads to the shortening of o-Ps lifetime $\tau_{3}=1 / \lambda_{3}$ :

$$
\lambda_{3}=\lambda_{p o}+\lambda_{T}
$$

where $\lambda_{p o}$ is the pick-off decay rate. The pick off process influences also the lifetime of para-Ps:

$$
\lambda_{1}=\lambda_{s}+\lambda_{p o}
$$

The value of $\lambda_{p o}$ for para- state, according to Dupasquier, is the same as for ortho state [31]. Due to the high value of $\lambda_{s}=8 \mathrm{~ns}^{-1}$ the role of pickoff in this case is marginal.

Positronium in condensed matter locates usually at "free volumes". i.e. electron-free regions: local voids, cavities, intermolecular empty spaces etc. In the single crystals of simple compounds: ice [32], silica (quartz) [33], with low intensity in alkali-halides [34] a delocalised Ps in a Bloch state was observed too (see Sec. 3.2).

When Ps is localised in a void, the probability of the pick-off process depends on the overlap of its wavefunction with the surrounding bulk medium. The larger the free volume, the smaller is the pick-off rate and hence the longer the o-Ps lifetime. Details of the relation between void size and o-Ps lifetime are described in Sec. 5.

Ortho-Ps in solid can be trapped at sites of various size, thus it can produce several long-lived components, therefore the summation in Eq. 2 should be made over all of them (i.e. the number of spectrum components is $n+2$, where $n$ means the number of different Ps trapping sites) ${ }^{2}$.

\footnotetext{
${ }^{2}$ The source envelope adds to the spectrum an extra component.
} 


\subsubsection{Spectrum analysis}

The analysis (decomposition) of a spectrum consists in determination of all $\lambda_{i}$ and $I_{i}$ and is performed using standard programs, like POSITRONFIT [35]. Sometimes the free volumes have no identical sizes, but (like in polymers) represent a continuous distribution around an average value. In such a case the LT program [36] can fit to the experimental spectrum, beside the average lifetime, also the width $\sigma_{i}$ of the lifetime distribution approximated as log-Gaussian one.

In the programs mentioned above the number of components is fixed a priori. In the MELT program [37] the approach is entirely different. A dense grid of several hundreds (or more) mean lifetime values is assumed and the result is a set of intensities ascribed to each point of the grid, the principle of statistical entropy maximum is used as a regularization factor. The quasi-continuous distribution of meanlifetimes can have an arbitrary shape; if it consists of distinct peaks, one can calculate the average mean lifetime and intensity for each peak. An example of the result of MELT calculations is shown in Fig. 7.

In typical cases the spectrum containing $10^{6}$ coincident events is sufficient for three-component constraint-free analysis; it can be collected in approximately $1 \mathrm{~h}$. The spectra for the MELT treatment need the statistics at least by one order of magnitude larger.

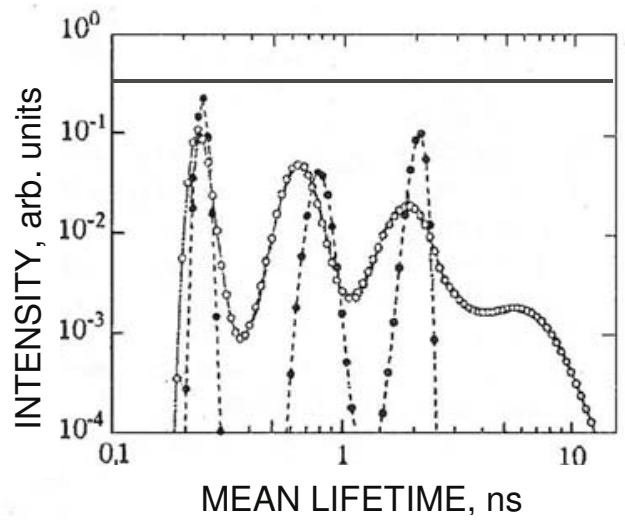

Fig. 7. Discrete and quasi-continuous lifetime spectra. Distribution of mean lifetimes obtained by the MELT procedure for poly(methylphenyl-silylenemethylene) sample. Dots - fresh sample; open circles after rapid cooling to $77 \mathrm{~K}$ and 118 h storage. (After Suzuki T. et al. [38]).

\subsection{Momentum distribution of annihilating pairs}

\subsubsection{Angular correlation of annihilation radiation (ACAR)}

In the coordinate system in which the $\mathrm{e}^{+} \mathrm{e}^{-}$pair before annihilation is at rest, the two-quantum annihilation results in the emission of two gamma quanta of the same energy $m_{e} c^{2}=511 \mathrm{keV}$ (exactly: $m_{e} c^{2}-B / 2$, 
where $B$ is the binding energy of an atomic electron) and in opposite directions. If the pair has certain momentum $p$, the direction of emission deviates from the angle $\pi$ by $\theta=p_{t} / m_{e} c$, where $p_{t}$ is the transversal component of the momentum and in such a case an angular distribution of annihilation quanta is observed. For typical momenta of electrons from outer atomic shells, the halfwidth of angular distribution is of the order of $0.5^{\circ}(10 \mathrm{mrad})$.

Let us choose the $x$ axis along the direction of one of quanta. The transversal component can be arbitrarily oriented in $y z$ plane (components $\left.p_{y}, p_{z}\right)$. The arrangement measuring the angular distribution of quanta consists of two scintillation counters working in coincidence. Single channel analyzers select from the amplitude spectrum the pulses representing the full energy peak $511 \mathrm{keV}$. One of the counters is fixed, the other moves in the direction, say, $z$ (Fig. 8).

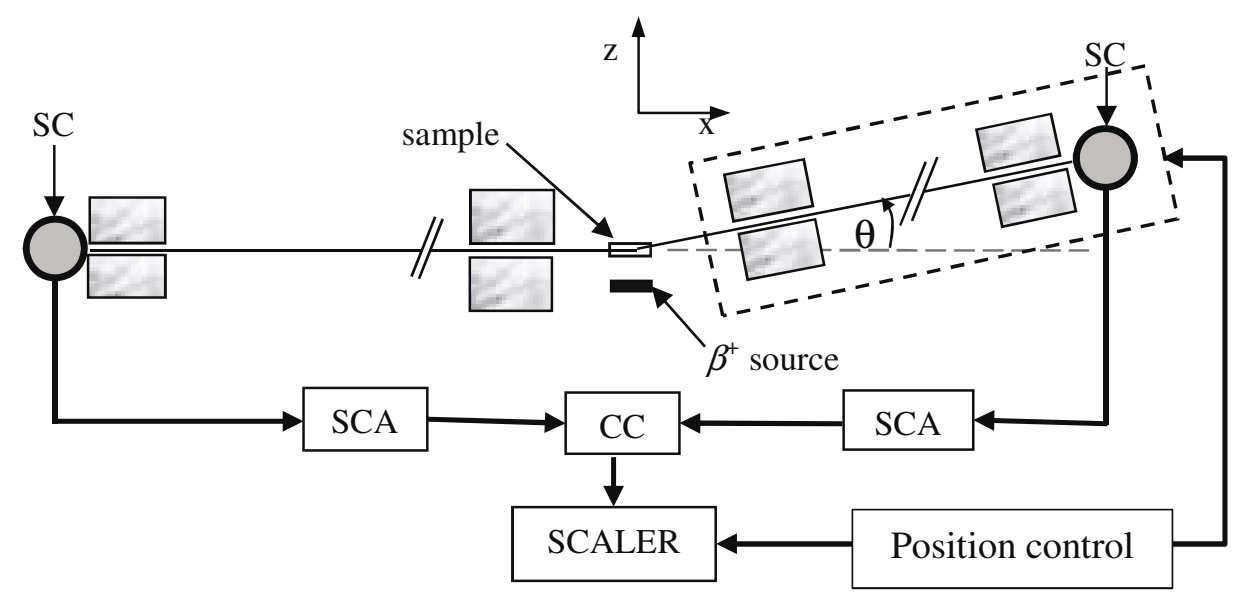

Fig. 8. Experimental set-up for the measurement of angular distribution of annihilation quanta (one-dimensional version). SC - the scintillation counter, SCA - the single channel analyser; CC - the coincidence circuit. HV supplies and (possible) amplifiers not shown.

The length of scintillators in the direction $y$ is made much larger than the expected span of the angular distribution, thus the coincidences are registered independently on the value $p_{y}$ (and also of $p_{x}$, because the energy resolution of scintillation counters is too low to notice the Doppler shift of $511 \mathrm{keV}$ quanta induced by the $p_{x}$ component). The angular distribution is very narrow, thus the angle subtended by the scintillators must be reduced by placing in front of them the lead slits parallel to $y$ axis, usually less than $1 \mathrm{mrad}$ wide. Thus, such an experimental 
arrangement registers the distribution of one momentum component only, $p_{z}$ (1D ACAR, "long-slit geometry"). If the medium is isotropic, one can transform the distribution of the $p_{z}$ component, $N\left(p_{z}\right)$, into the distribution of full momentum:

$$
N(p)=-p_{z} \frac{d N\left(p_{z}\right)}{d p_{z}}
$$

where $p_{z}=m_{e} c \theta$.

At the moment of annihilation free positrons are thermalized, thus their share in the total momentum of $\mathrm{e}^{+} \mathrm{e}^{-}$pair is negligible and the pair momentum is determined almost exclusively by the electron. Due to the Coulomb repulsion of positrons by nuclei the annihilation occurs mainly on outer, valence electrons producing the main component of the width of the order $10 \operatorname{mrad}\left(p \approx 10^{-2} m_{o} c\right)$. A broader distribution of annihilation on inner shell electrons is of very low intensity and in most cases merges with background.

The thermalized para-Ps annihilates as a whole, thus it should produce a "narrow component" of the width corresponding to the thermal energy (also convoluted with instrumental resolution determined by the slit width and thus slightly broadened). Such a narrow peak in the angular distribution is observed in the case of delocalized positronium in a Bloch state. Beside the peak around $p=0$, the momentum spectrum contains also some sidepeaks located at $\vec{p}+\vec{G}$, where $\vec{G}$ is the reciprocal lattice vector. Usually, Ps in solids is trapped in a limited volume and the width of the $p$-Ps momentum distribution is ruled by the uncertainty principle. In such, most common, case this component is (3-5) mrad wide (Fig. 9), but traditionally still named "narrow" one.

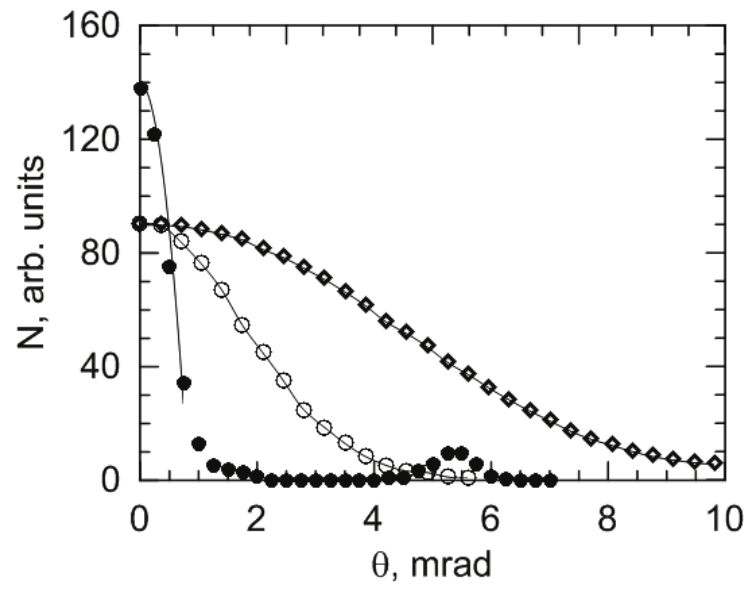

Fig. 9. Angular distribution of two-gamma annihilation radiation. Dots - delocalized para-Ps component in ice single crystal, the peak at $5.6 \mathrm{mrad}$ corresponds to $G_{z}$ component of reciprocal lattice vector [32]; circles - paraPs and diamonds - free positron annihilation components in p-terphenyl doped with anthracene. 


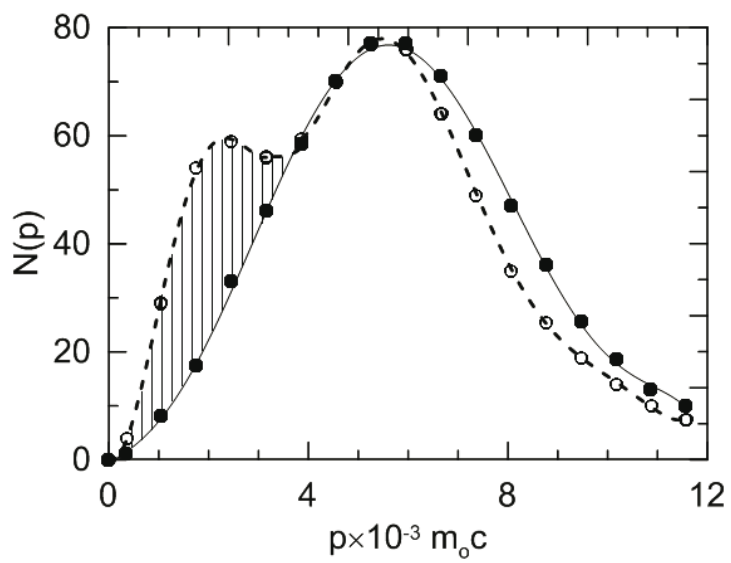

Fig. 10. Momentum distri-bution $N(p)$ of annihilating pairs in solid (dots, no positronium) and liquid (empty circles, $70 \%$ of positronium) guaiazulene [40]. The fragment belonging to para-Ps - dashed.

The ortho-Ps decaying via three quantum emission is not visible in ACAR (it is not peaked at $180^{\circ}$ and has a continuous energy spectrum). A two-quantum pick-off process, involving electrons from molecular bonds, should give a momentum distribution similar to that of free positron annihilation. It was found however, that these two distributions are not identical; usually the pick-off component is slightly narrower, e.g. in liquid benzene the widths for pick-off and for free annihilation are $7.25 \mathrm{mrad}$ and $9.57 \mathrm{mrad}$, respectively [39]. Fig. 10 shows the full momentum distribution (Eq. 5) for solid and liquid guaiazulene [40]. For a solid sample $N(p)$ represents the annihilation of free positrons (no Ps); in liquid state a strong p-Ps component appears, the remainder is the sum for free and o-Ps pick-off annihilation, which is narrower compared with the annihilation of free positrons.

\subsubsection{Two-dimensional momentum analysis}

Recently, the one-dimensional ACAR devices are rarely used; single scintillation counters are substituted by position sensitive detectors of the size much larger than the width of pair momentum distribution (up to 50x50 $\mathrm{cm})$. In such an arrangement two components of pair momentum $p_{\mathrm{y}}, p_{\mathrm{z}}$ can be determined simultaneously (2D ACAR). The detectors can be Anger cameras [41] or Charpak wire-chambers with Jeavons gamma converters [42] placed in front of them [43]. The resolution obtainable with Anger cameras is $(3 \div 5) \mathrm{mm}$, with wire chambers $-(1 \div 1.5) \mathrm{mm}$, which at the distance sample - detector of $\approx 10 \mathrm{~m}$ assures an angular resolution of 
$0.25 \mathrm{mrad}$. The positron sources used in ACAR measurements are $(10 \div 50)$ $\mathrm{mCi}$, thus by three orders of magnitude stronger than in PALS measurements.

The 2D ACAR technique is used in probing the crystal structure and defects by positrons, not positronium, and is mentioned here for completeness only.

\subsubsection{Doppler broadening of $511 \mathrm{keV}$ annihilation radiation line (DBARL)}

The longitudinal component of the pair momentum $p_{x}$ causes the Doppler shift of both annihilation quanta. The conservation of that component gives:

$$
\frac{h(v+\Delta v)}{c}-\frac{h(v-\Delta v)}{c}=p_{x}
$$

thus, the Doppler shift of quantum energy is:

$$
\Delta(h v)=\frac{c p_{x}}{2}
$$

If the momentum distribution is isotropic, the distribution of energy in the annihilation line is identical with that of angular distribution of coincident $2 \gamma$ quanta. For the energy of outer electrons in atoms $E_{a t}$, the corresponding maximal $\left(p_{x}= \pm p\right)$ Doppler shift of an annihilation quantum is:

$$
\Delta(h v)= \pm \frac{c \sqrt{2 m_{e} E_{a t}}}{2}= \pm 506 \sqrt{E_{a t}}
$$

(in electronvolts). The energy shift by $1 \mathrm{keV}$ is equivalent to $3,914 \mathrm{mrad}$ in angular distribution $\left[\theta=2 \Delta(h v) / m_{e} c^{2}\right]$. The best resolution obtainable with high purity germanium (HPGe) detectors is (1.0-1.2) keV FWHM for the $511 \mathrm{keV}$ line. Thus, the profile of a Doppler line is the same as an angular distribution taken with a resolution of (4-5) mrad, in comparison to usual $1 \mathrm{mrad}$ (or less) in ACAR technique.

When the positron source is ${ }^{22} \mathrm{Na}$, the annihilation line in the spectrum registered by the detector is superposed on the background of $1274 \mathrm{keV}$ gamma ray Compton continuum and to facilitate the analysis of Doppler profile this background should be subtracted (see next section).

In the early stage of DBARL technique, several attempts were undertaken to deconvolute the finite resolution of the detector and to reconstruct the true momentum distribution [44]. Currently, a simplified, qualitative approach is in use [45]. The $\gamma$-quantum energy distribution is 
characterized by two parameters: $\mathrm{S}$ which is the ratio of area under central part of annihilation peak to the total area under that peak, and $\mathrm{W}$ - the ratio of areas selected on the wings of the peak to the total area (Fig. 11) ${ }^{3}$. These parameters are sufficient if one wants to observe the changes in the spectrum only; e.g. increase of p-Ps intensity is seen as the increase of $S$ parameter. The widths of line fragments, used in $\mathrm{S}$ and $\mathrm{W}$ definition, are arbitrary, but in common practice $\mathrm{S}$ contains about $50 \%$ of line area, $\mathrm{W}$ - about $(6 \div 8) \%$.

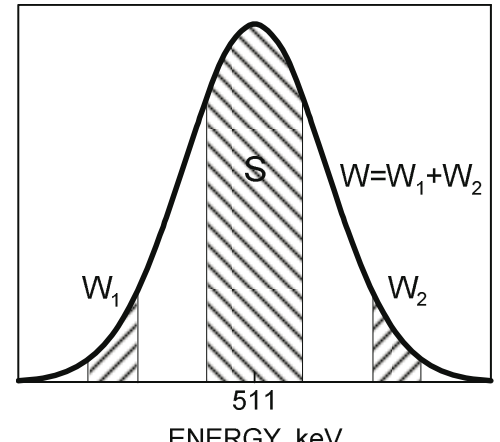

ENERGY, keV
Fig. 11. $\mathrm{S}$ and $\mathrm{W}$ parameters mean the ratios of the areas in selected range of energy to the total area of the $511 \mathrm{keV}$ peak.

Operating the $\mathrm{S}$ and $\mathrm{W}$ parameters is commonly practicized in the cases when no positronium in the sample appears (metals, alloys); e.g. the plot in the coordinates S vs. W allows to identify the defects in the sample structure. The attempts to improve the deconvolution method are continued till now [46].

\subsubsection{Coincidence Doppler spectrometry}

The background arising from the Compton scattering of the 1274 $\mathrm{keV}$ gammas can be eliminated by registering the spectrum gated by the full energy pulses $1274 \mathrm{keV}$. A schematic diagram of an experimental setup is shown in Fig. 12a. The germanium detector registers the annihilation radiation spectrum, the scintillation counter (of much higher efficiency) - the gamma quanta of $1274 \mathrm{keV}$. In this version the whole spectrum of pulse amplitudes related to the interaction of the $511 \mathrm{keV}$ quanta with the detector is recorded, including the Compton continuum (see Fig. 2A).

\footnotetext{
${ }^{3}$ Parameters S and W were used by W. Gustaw and K. Zaleski still in 1980 [47], in the positron study of titanium alloys and steel.
} 
a)

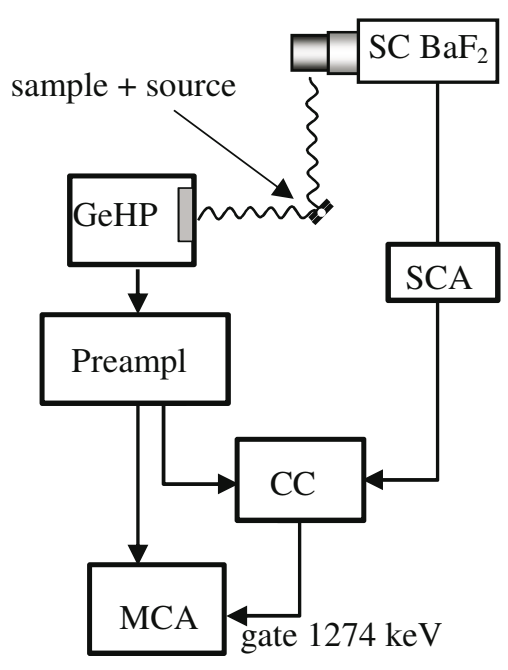

b)

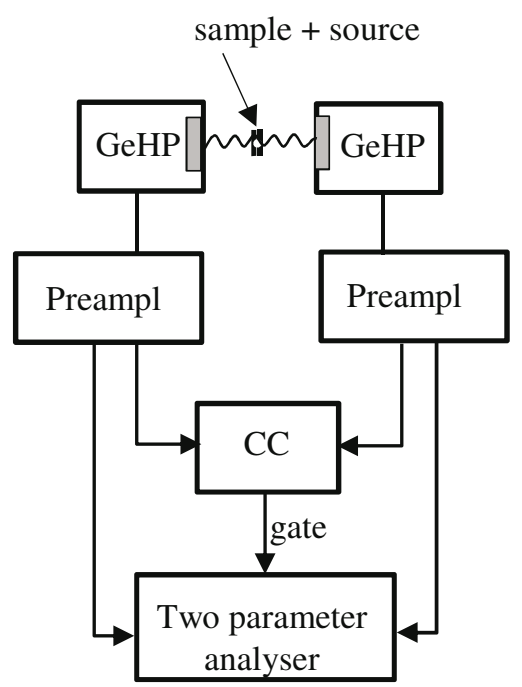

Fig. 12. a) DBARL coincidence spectrometer with background reduction; b) two-dimensional DBARL spectrometer $511-511 \mathrm{keV}$.

The lower part of the left side of the full energy $511 \mathrm{keV}$ peak is slightly distorted, because on that side the counting rate does not decrease to zero due to the presence of pulses produced at multiple Compton scattering.

Another version of coincidence Doppler spectrometer is a twodimensional one (Fig. 12b). Two germanium detectors placed head-tohead with the sample and source between them work in coincidence and the amplitudes of pulses are recorded in a rectangular matrix - the horizontal axis is for the amplitude of pulses from one counter, the vertical one - from the other counter [48]. A typical picture from this kind of spectrometer is shown in Fig. 13. Placing the counters at a certain distance from the source $(15-25 \mathrm{~cm})$ one can reduce further the background from the Compton continuum of $1274 \mathrm{keV}$ gamma rays. Along the diagonal on the diagram like Fig. 13 the Doppler shift in one counter is accompanied by an opposite shift in the other counter, thus the difference of energies of two quanta is equal to the doubled Doppler shift:

$$
E_{\gamma 1}-E_{\gamma 2}=c p_{x}
$$




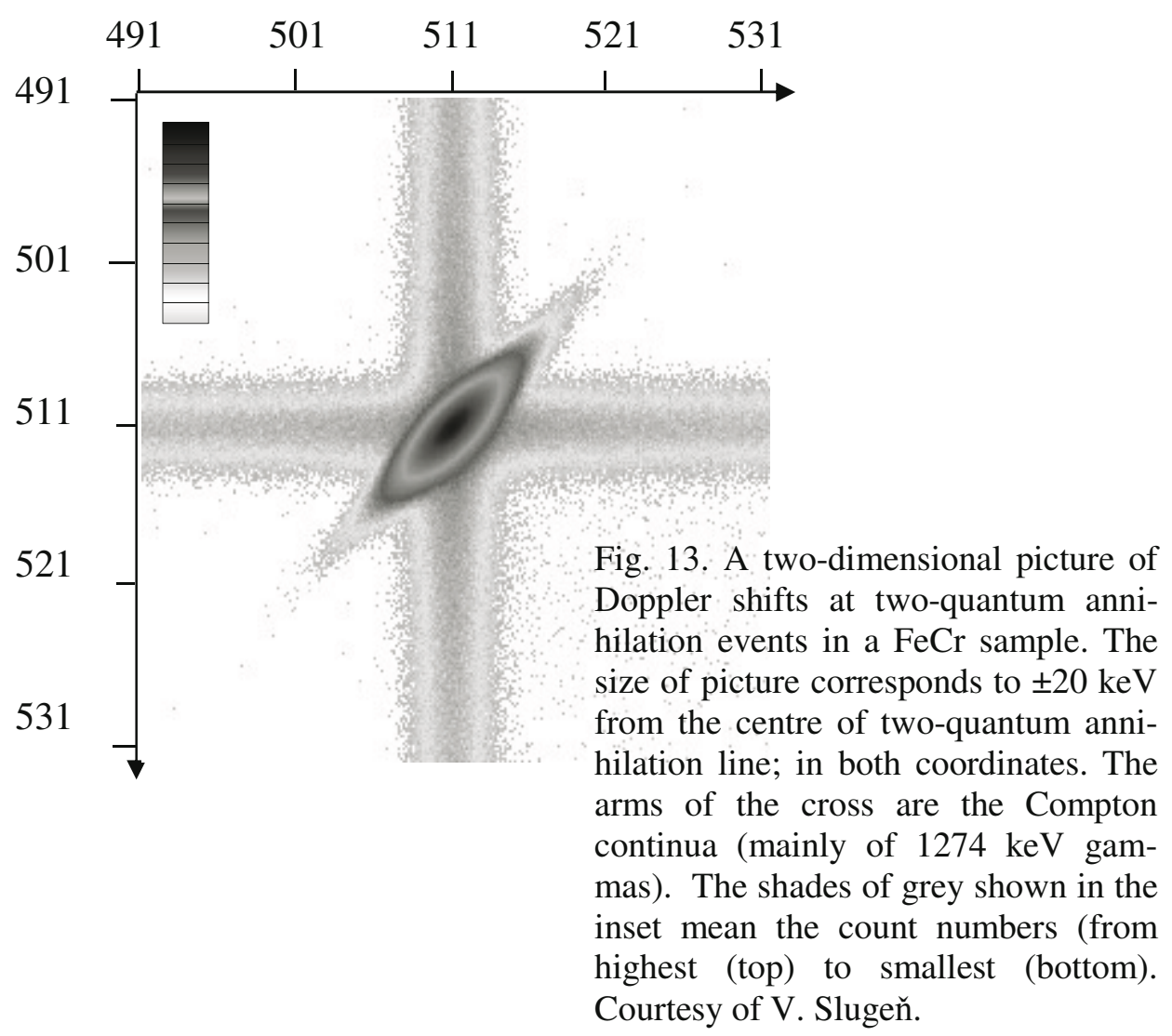

Owing to the doubled shift, the momentum resolution is improved by a factor of $\sqrt{2}$. The spectrum recorded along the diagonal is practically without background (peak to background ratio about $10^{5}: 1$ ) and gives the opportunity to observe the momentum distributions of electrons from inner shells in spite of very low their contribution.

\subsection{Age-momentum correlation method (AMOC)}

The method consists in simultaneous recording the lifetime and momentum of annihilating pair. In early years of positron spectroscopy, when the momenta were measured by the ACAR method (i.e. the angle was changed step by step) it was possible to record the set of lifetime spectra for several selected angles $\theta$ only [49]. The advent of germanium detectors enabled to perform real two-parameter measurements [50]. The start signal is produced by a scintillation counter $\left(\mathrm{BaF}_{2}\right.$ when the positron 
source is ${ }^{22} \mathrm{Na}$, or a thin plastic when a high energy positron goes through). The stop $511 \mathrm{keV}$ origins from other $\mathrm{BaF}_{2}$ being in coincidence with a third counter, HPGe, registering the spectrum of annihilation quanta emitted in opposite direction to those which fell into the stop $\mathrm{BaF}_{2}$ counter.

In the two-parameter spectrum one axis is for the lifetime, the other for the energy of annihilation radiation. An example of a two-dimensional spectrum for fused quartz is shown in Fig. 14.

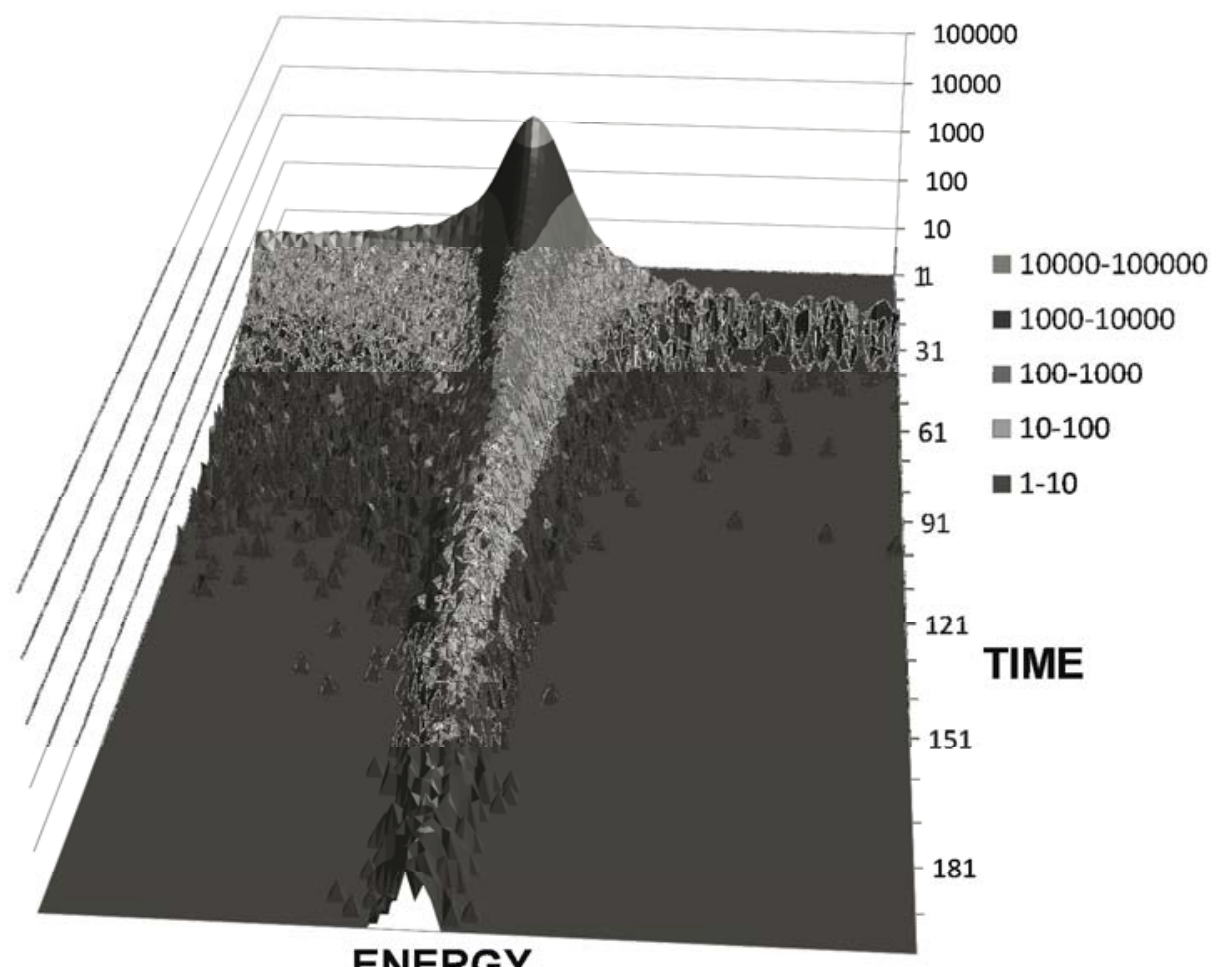

Fig. 14. A two-parameter picture obtained by age-momentum correlation method - fused quartz. Time units on the right of picture are $80 \mathrm{ps.} \mathrm{(courtesy} \mathrm{of}$ T. Hirade).

Usually the data from AMOC experiments are presented as cross sections of a three-dimensional picture. At a fixed delay the Doppler broadened $511 \mathrm{keV}$ peak describes the momentum distribution. For a given delay time $t$ the Doppler profile allows to determine the $S$ parameter and the set of data is displayed as a function of positron age (delay), $S(t)$. Alternatively, at a fixed gamma energy one can calculate the 
average lifetime $\tau_{\mathrm{M}}{ }^{4}$ and draw it as a function of energy: $\tau_{\mathrm{M}}$ vs. $E_{\gamma}$ ("Tsukuba plot" [51]). A typical $S(t)$ plot for the case of sample in which positronium is formed is shown in Fig. 15.

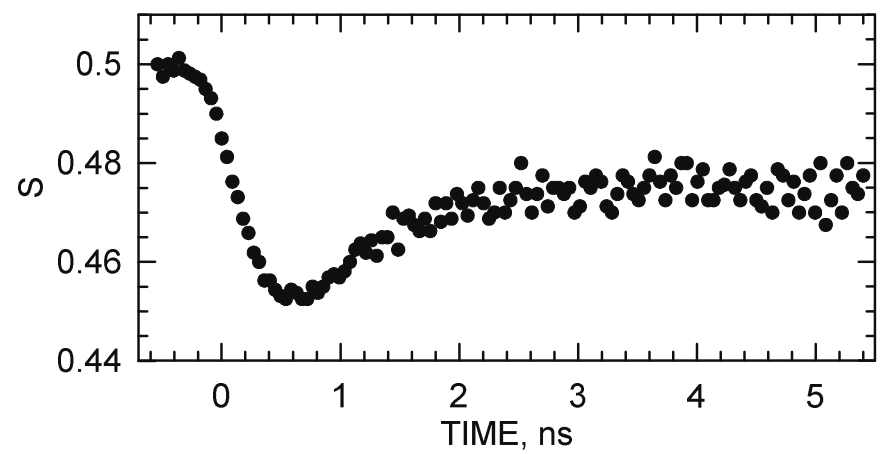

Fig.15. Typical dependence of S parameter on the positron age, as obtained by the AMOC method.

At the beginning of time scale the value of $S$ is large owing to the narrow p-Ps component, then dominant is the free annihilation with its broadest momentum distribution (small $S$ ), finally, at larger delays the momentum spectrum belongs to the decay of o-Ps with slightly smaller momenta.

\subsection{Three-quantum annihilation}

Positrons, which have not formed positronium and are moving in the medium as free particles, annihilate mainly with the emission of two gamma quanta. The fraction $f_{3}$ of positrons annihilating via $3 \gamma$ decay is determined by the ratio of cross sections for the respective annihilation modes, $f_{3}=\sigma_{3 \gamma} / \sigma_{2 \gamma}$.

$$
\begin{gathered}
\sigma_{2 \gamma}=\pi r_{0}^{2} c / v \\
\sigma_{3 \gamma}=\frac{4\left(\pi^{2}-9\right)}{3 v} r_{o}^{2} c \alpha
\end{gathered}
$$

where $r_{0}$ is the classic electron radius $\left(r_{0}=2.82 \cdot 10^{-13} \mathrm{~cm}\right), \alpha=1 / 137$ the fine structure constant, $v$ - the positron velocity. The ratio of cross sections is $1 / 372$; i.e. $0.27 \%$ of free positrons annihilate via $3 \gamma$ process. When Ps atom is formed, ortho-Ps in vacuum should annihilate with the emission of 3 gamma quanta only, however the majority of o-Ps disappears via the two-

\footnotetext{
${ }^{4} \tau_{\mathrm{M}}$ means the average over whole time spectrum.
} 
quantum pick-off process. The fraction of o-Ps decaying with $3 \gamma$ emission is equal to the ratio of decay constants in vacuum and in the medium. The relative intensity of $3 \gamma$ decays is then:

$$
f_{3}=\frac{(1-P)}{372}+I_{o-P s} \frac{\tau_{3}}{\tau_{3}^{0}}
$$

where $P$ is the probability of Ps formation, $P=I_{p-P_{s}}+I_{o-P s} ; I_{p-P_{s}}$ and $I_{o-P_{s}}$ are the relative intensities of para and ortho components in the spectrum, $\tau_{3}$ is the lifetime of o-Ps in the sample, $\tau_{3}{ }^{0}$ - in vacuum (para-Ps does not participate in the $3 \gamma$ process). If there are no chemical interactions at positronium formation $I_{o-P S} / P$ should be $3 / 4$, according to the statistical weights of the Ps states.

The simplest way to determine the fraction of the $3 \gamma$ annihilation events is to measure the reduction of $511 \mathrm{keV}$ peak intensity in the gamma spectrum observed with a germanium detector, compared to a "no-Ps containing" standard sample. If the positron source is ${ }^{22} \mathrm{Na}$, one can normalize the spectra to the same area under the $1274 \mathrm{keV}$ peak, which is equivalent to the same number of $\beta^{+}$decays. The energy spectrum of $3 \gamma$ annihilation is continuous, roughly triangular in shape, with a sharp edge at $511 \mathrm{keV}$. Thus, the counts related to it are dispersed over the whole energy spectrum; this way they disappear from the $511 \mathrm{keV}$ peak, thereby reducing its intensity. One should remember that any Ps-free standard contains $0.27 \%$ of three-gamma events originating from the decay of free positrons. The high energy edge of the $3 \gamma$ spectrum is not smeared out by Doppler effect, because in the three-gamma process the whole object annihilates with negligible momentum.

One can determine the $3 \gamma$ annihilation intensity via the counting rate of triple coincidences in 3 scintillation detectors placed around the sample with positron source. If the angular spacings of the counters are equal, $120^{\circ}$, the annihilation quanta registered by these counters have the energies $341 \mathrm{keV}$ and pulse amplitude selectors should be set to this energy (Fig.16). The emitted quanta are co-planar (with an accuracy of $\sim 0.5^{\circ}$, like the co-linearity in two quantum annihilation), thus the random coincidence background can be determined by moving one counter out of the plane determined by the two others and the sample. The $3 \gamma$ fraction $f_{3}$ is usually determined by comparing the observed rate for the sample under study to that rate in a medium in which Ps does not form, e.g. a metallic one, or better, the medium with high but well known $3 \gamma$ intensity. (the both spectra should be measured using the same source, geometry and with similar absorption coefficient of annihilation quanta). 


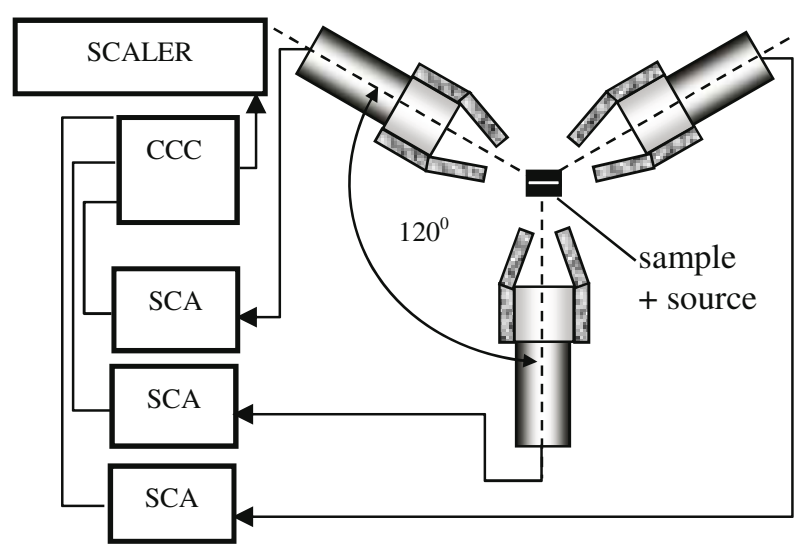

Fig. 16. Experimental setup for registration of three-gamma annihilation events.

One can evaluate the $3 \gamma$ fraction from the equation (12) if the relation between $I_{\mathrm{oPs}}$ and the intensity of the long-lived component in a PALS spectrum $I_{3}$ is known. Applying the lifetime spectroscopy to determine $f_{3}$ one has to set a very broad energy window in the STOP channel of the

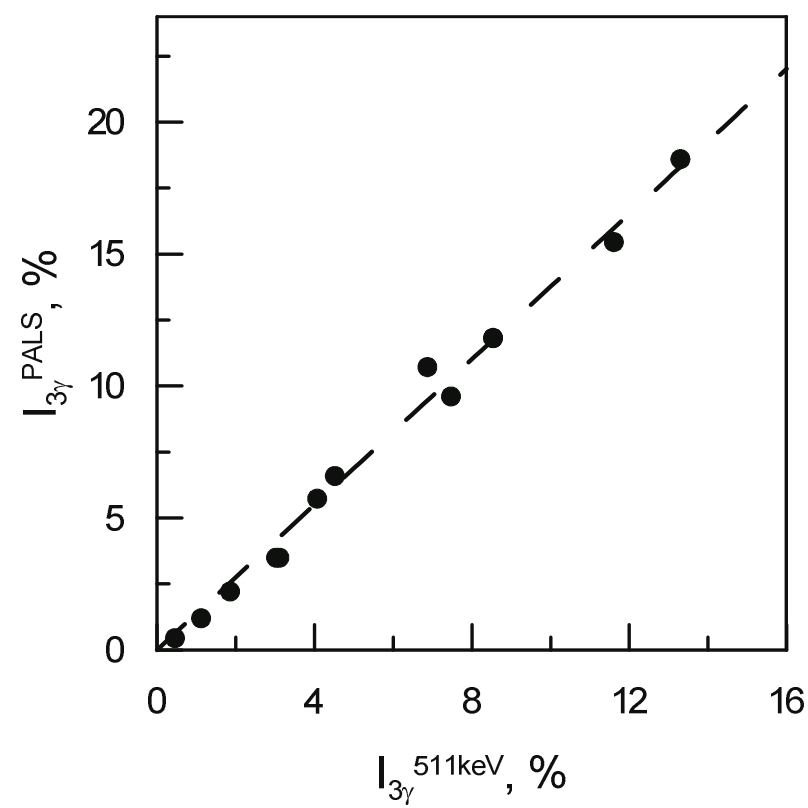

Fig. 17. A comparison of $\mathrm{I}_{3 \gamma}$ intensities determined from the reduction of the $511 \mathrm{keV}$ peak intensity and by PALS with very broad energy window in the spectrometer. Data collected for a set of porous Vycor glass samples (R. Zaleski, Ph.D. Thesis, UMCS Lublin 2005) spectrometer. Usually one assumes $I_{3}=I_{\mathrm{oPs}}=$ $3 / 4 P$; this would be valid when the efficiency of registration of all gamma quanta was identical. However, the lower the quantum energy, the higher the efficiency of its registration, thus one can expect $I_{3}>3 / 4 P$. On the other hand, a part of $3 \gamma$ events is lost as the energy window in the STOP channel cannot reach down to zero energy. At a very broad window covering the low energy part of the spectrum the $3 \gamma$ fraction is usually overestimated. Fig.17 shows the com- 
parison of $I_{\mathrm{oPs}}$ determined from the reduction of the $511 \mathrm{keV}$ peak intensity and $I_{3}$ found from the decomposition of a PALS spectrum.

Finally, as a measure of the $3 \gamma$ decay fraction one can use the ratio of intensities in selected regions of the energy spectrum: in the region between the $511 \mathrm{keV}$ full energy peak and the Compton continuum to that in the region of the $511 \mathrm{keV}$ peak ("valley to peak ratio"). A continuous spectrum of $3 \gamma$ decays increases the counting rate in the "valley". In the classic measurements with a ${ }^{22} \mathrm{Na}$ source the intense background of Compton continuum belonging to the $1274 \mathrm{keV}$ gamma line makes this kind of $f_{3}$ estimate difficult. Much easier is such a measurement with positron beam, which produces the annihilation radiation only.

\section{POSITRONIUM FORMATION AND TRAPPING}

\subsection{The Ore model and blob model}

A simple mechanism of Ps formation was proposed by Ore [52]. It is described as one-act process and seems to be suitable for gaseous media at low pressure. As long as the positron energy is larger than the ionization potential $E_{i}$, or even the lowest electronic excitation potential $E^{*}$, the chances of the reaction:

$$
\mathrm{e}^{+}+\mathrm{M} \rightarrow \mathrm{Ps}+\mathrm{M}^{+}
$$

(M means an arbitrary molecule) are negligible. At the moment of binding $\mathrm{e}^{+}$and $\mathrm{e}^{-}$particles the energy of $6.8 \mathrm{eV}$ is released, thus the minimal positron energy necessary to Ps formation is $E_{\mathrm{i}}-6.8 \mathrm{eV}$; this is an endothermic process. Only the positrons from the range $\left(E^{*}, E_{i}-6.8 \mathrm{eV}\right)$ are able to form Ps. This range is called „Ore gap”. If the positron energy distribution is uniform from 0 to $E^{*}$, the probability of Ps formation is:

$$
P=\left(E^{*}-E_{\mathrm{i}}+6.8 \mathrm{eV}\right) / E^{*}
$$

In the condensed matter the processes are much more complex; it is necessary to account radiation chemistry effects. The first step on this way of problem treatment was made by Mogensen [53] and Byakov [54], the idea was further developed in the papers by Tao [55], Ito [56] and Stepanov [57, 58].

Except the case of experiments with slow positron beams, the energy of positrons is much higher than the ionization potential of atoms in the medium which it penetrates. A high energy positron ionizes the medium; 
in the most simplified version of the model, at the ionization place we have one electron ${ }^{5}$, one positron and one ion (such a set is called spur), electron recombination with an ion competes with Ps formation, without any threshold energy (contrary to the Ore model). The chances of Ps creation depend on the distance $d$ between $\mathrm{e}^{+}$and $\mathrm{e}^{-}$and the radius of Onsager sphere $r_{\mathrm{c}}$, i.e. the distance of two elementary charges at which their energy of Coulomb interaction is equal to the average energy of thermal motion $k T$ :

$$
r_{c}=e^{2} /\left(4 \pi \varepsilon_{o} \varepsilon k T\right)
$$

where $\varepsilon$ is the dielectric permittivity (the particles are assumed as thermalized). The electron has a choice between two positive charges, thus the Ps formation probability is [55]:

$$
P=\frac{1}{2}\left[1-\exp \left(-r_{c} / d\right)\right] \exp \left(-\lambda_{2} \tau_{P_{s}}\right) \approx \frac{1}{2}\left\{1-\exp \left(-r_{c} / d\right)\right]
$$

The second exponential factor accounts for the possibility of positron annihilation as a free particle before binding into Ps. The positronium formation time $\tau_{\mathrm{Ps}}$ is short, particularly in liquids. In solid hydrocarbons the simulation by Garcia et al. [59] estimates that time as 20 ps , thus this exponential can be assumed close to unity. In liquids the Ps formation probability strongly depends on solvatation processes. A solvated electron or positron has a drastically reduced mobility and therefore the probability of binding both particles into Ps becomes greatly diminished. In particular, the electron (positron) solvatation time in water is very short, below one picosecond [60].

This "spur model" was further modified. For typical non-polar media $\varepsilon$ is about 2 , and at room temperature $r_{c}$ is approximately $30 \mathrm{~nm}$, on the other hand, the distance at which the low energy $(<500 \mathrm{eV})$ positron changes its direction of motion, due to the collisions, by more than $90^{\circ}$ (the path of transport) is a fraction of nanometer. Due to those frequent changes of the direction of motion there is more than one electron and one ion in the vicinity of the last ionization place, thus at the end of track we have no simple spur, but a structure rich in particles called blob (Fig. 18). The blob disappears quickly, after electron - cation radical recombination or outdiffusion of various blob elements, a pair consisting of electron and positron can remain. If their distance is less than $r_{c}$ they cannot escape

\footnotetext{
${ }^{5}$ In the ionization act electron can receive the energy sufficient to cause secondary ionizations. When that energy is high such electron forms own ionization track ( $\delta$-electron).
} 
each other and perform a diffusive correlated motion through the medium (free quasi-positronium, qPs).

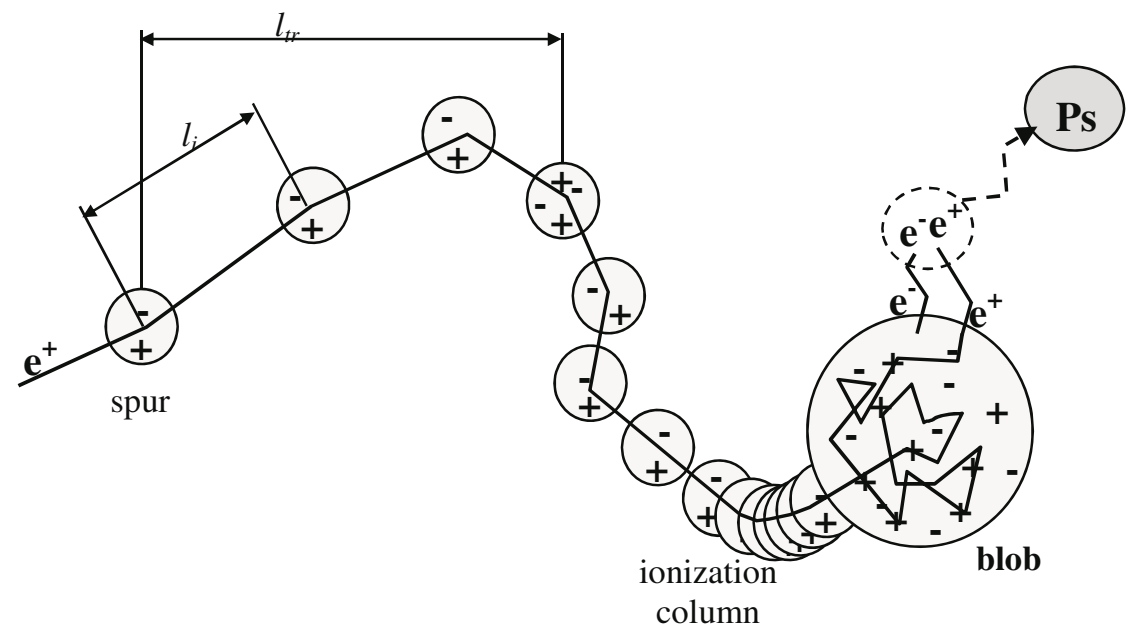

Fig. 18. Positron track in condensed matter. $l_{i}-$ the path of ionization, $l_{t r}-$ the path of transport (the size of objects does not correspond to real proportions).

In vacuum the Ps binding energy is $6.8 \mathrm{eV}$; after the collapse of the blob we have a pair whose energy is composed of the $\mathrm{e}^{-}$energy (polarization of the medium, kinetic energy etc.), the $\mathrm{e}^{+}$energy (as above) and the energy of Coulomb interaction of these charged particles. Usually, the sum of these energies is less than $6.8 \mathrm{eV}$. The boundary bulk - vacuum represents then an energy step of the height $U$ equal to the difference of pair energy in the bulk and outside, in vacuum, thus corresponds to the Ps work function. If the $\mathrm{e}^{+} \mathrm{e}^{-}$pair is near the surface and $U$ is negative, Ps formation is energetically favourable, Ps would be emitted to the vacuum with the kinetic energy equal to that work function.

If the region of "vacuum" is limited in all directions, it represents a void, being a potential well of the depth $U$. The energy balance is now slightly modified; on the side of void a positive term - the zero-point energy of trapped Ps appears. The centres of Ps formation are thus the empty volumes (or at least of negligible electron density) inside the bulk: voids, cavities.

To be trapped in the potential well, Ps needs to lose some energy at entering the void, or at hitting the opposite wall and settle on a certain energy level in the well, otherwise it will go through the well untrapped. 
The energy loss occurs via phonon creation or by excitation of internal oscillations of the molecular bonds.

In the case of solids the value of $U$ can be determined by the time of flight (TOF) method [61]. A schematic diagram of an experimental equipment is shown in Fig. 19. The pulsed beam of slow positrons is directed onto the sample; positronium atoms ejected from the sample pass by the lead slits with inserted sheet-like scintillators. Ps atoms decay in flight, some of them when in front of the slit. The integrated current signal in the scintillation counter is registered as a function of the time since the sample is hit by the incident positron bunch. The delay of signal is equal to the time of flight of Ps and for given base length allows to determine the velocity and the kinetic energy of o-Ps. The inset on the right of figure shows the results of TOF measurements by Nagashima et al.[62] for KJ crystal. The values of $U$ found in the experiments of this kind lie in the range $1 \div 3 \mathrm{eV}$.

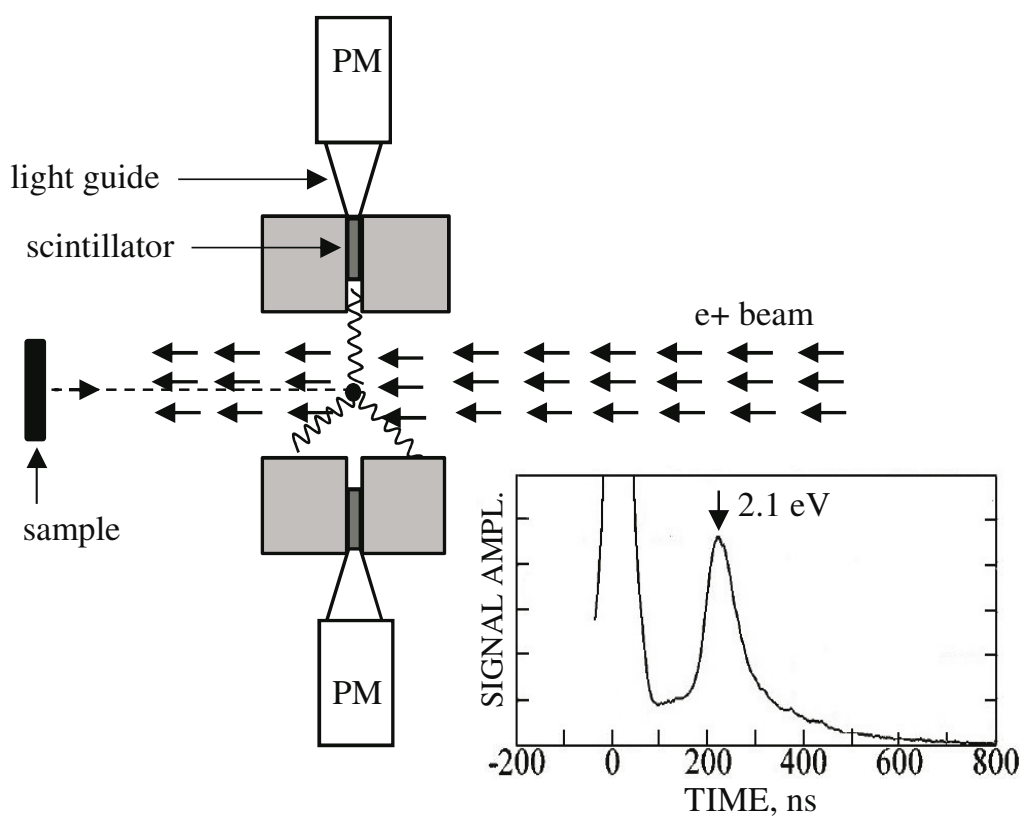

Fig. 19. Experimental setup for time of flight (TOF) method measurements (PM - photomultiplier). Inset: example of the current in the photomultiplier registering the decays of o-Ps emitted from potassium iodide crystal (after Ref. 62).

The spur (blob) model is actual in the case of natural $\mathrm{e}^{+}$sources; large energies assure the appearance of many ionization events. At low 
positron energy (slow beams) the process of Ps formation is reduced to single positron-molecule interaction, i.e. the Ore model works. It was demonstrated by Eldrup et al. [63] observing the emission of Ps from the surface of ice bombarded by slow positrons. In the solid the upper $E_{U}$ and lower $E_{L}$ limits of Ore gap are:

$$
\begin{gathered}
E_{U}=E^{*}-Q^{+}, \\
E_{L}=E_{i}-6.8 \mathrm{eV}-U
\end{gathered}
$$

where $U$ and $Q^{+}$are the work-functions of Ps and positron from the solid, respectively. For ice $E_{i}=9.8 \mathrm{eV}, U$ is estimated as $(2 \pm 1) \mathrm{eV}$, thus the threshold of Ps formation $E_{L}$ is expected to be about $5 \mathrm{eV}$. The experiment confirms that value, the intensity of Ps emission reaches a maximum at about $6 \mathrm{eV}$ and then decreases by a factor of two - the positron energy exceeds already the upper limit of Ore gap.

\subsection{The shape of potential well}

Due to the short range of atomic interactions the radial dependence of the potential is usually assumed as rectangular (stepwise) and, for simplicity, the geometry of a void as spherical. An exactly rectangular shape of the potential is a simplification: the electron density does not change in an exactly stepwise form; more appropriate would be to assume the Saxon-Woods potential [64]:

$$
V(r)=U\left[1-\frac{1+\exp (-R / a)}{1+\exp [(r-R) / a]}\right]
$$

where $a$ describes the diffuseness of void limits. For $a<R$ that potential transforms into a rectangular one. In the literature $[65,66]$ one can find calculations where the shape of potential has the form like $\tanh ^{2} r$, or $[1-\exp (-r / R)]$, however such shapes seem to be unrealistic. In further discussions the rectangular potential will be used here as an approximation sufficiently accurate and easy in handling.

\subsection{The smallest detectable voids}

With reducing the "empty void" size the zero point energy of a confined particle rises like reciprocal of the square of linear void dimensions; the energy gain following from binding the pair diminishes. At a certain size, $R_{\min }^{\prime}$, the energy of the level for a particle approaches 
the upper rim of the well, at the radii $R$ below $R_{\min }^{\prime}$ there is no energy level for Ps in the well. In the case of a stepwise spherical potential the minimal void radius at which the energy level for Ps exists is:

$$
R_{\min }^{\prime}=\frac{\pi \hbar}{4 \sqrt{m_{e} U}}=\frac{0.216 n m}{\sqrt{U}}
$$

(for $U$ in $\mathrm{eV}$; positronium mass is $2 m_{e}$ ).

If one takes into account that Ps is not a structureless particle but a kind of hydrogen-like atom composed of two particles, there is another source of the lower limit of void radius needed for positronium. When an atom is confined in a spherical well its binding energy diminishes. This problem, for the case of hydrogen atom, was discussed by Sommerfeld and Welker as early as in 1938 [67] and applied to Ps by Bartenev et al.[68]. The wavefunction of hydrogen-like Ps in a rectangular (infinitely deep) well is:

$$
\psi(r)=A \exp \left(-\frac{\rho}{2}\right) W(\rho, n)
$$

where $\rho=2 r / n a, a$ is the electron-positron distance (doubled Bohr radius $\left.a_{0}\right)$ and $W(\rho, n)$ is the degenerate hypergeometric function which can be written in the form of series:

$$
W(\rho, n)=1+\sum_{k=1}^{\infty}(-1)^{k} \frac{\prod_{m=1}^{k}(n-m)}{(k+1) !} \frac{\rho^{k}}{k !}
$$

where $n$ is not an integer, its value follows from the condition that $W(\rho, n)$ has to be zero at the wall of the well, i.e. for $r=R$. The value of $n$ found from this condition enters the expression for the Ps atom binding energy:

$$
E_{P s}=\frac{m_{e} e^{4}}{16 \varepsilon_{0}^{2} n^{2}}
$$

The dependence of the binding energy on the void radius is shown in Fig. 20 [69]. This energy becomes zero at $R_{\min }^{\prime \prime}=0.194 \mathrm{~nm}$; at $R<R_{\min }^{\prime \prime}$ an atom-like structure cannot be bound. Free volumes of radii smaller than $R_{\min }^{\prime}$, or $R_{\min }^{\prime \prime}$ (or equivalent for geometries other than spherical), cannot be the centres of Ps formation and their presence in the sample is not noticed in positronium experiments. 


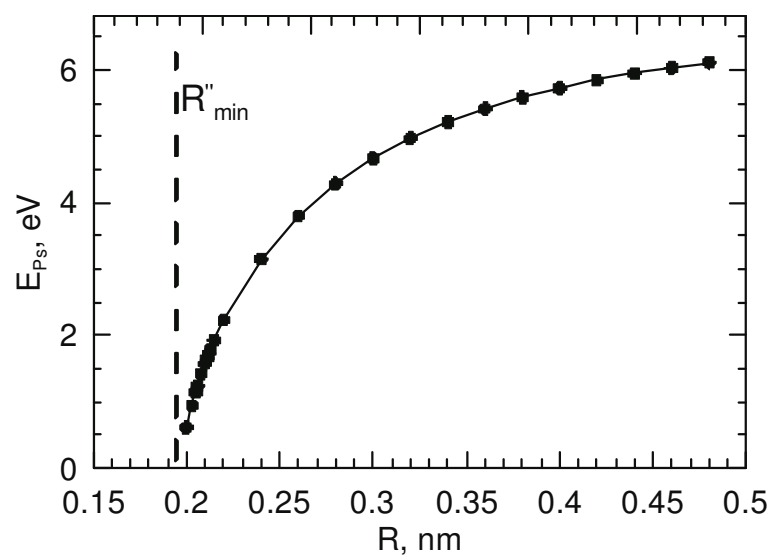

Fig. 20. Positronium binding energy as a function of the void radius (potential infinitely deep). Four terms of the series (20) are taken into account [69].

With increasing $R$ the binding energy rises rapidly, and already at $R_{\min }^{\prime \prime}=0.5 \mathrm{~nm}$ it is about $6 \mathrm{eV}$, thus close to the value in open space and the effect of confinement can be neglected. One has to remember that the calculations presented above are for an infinitely deep well, for a finite one the numbers can be different.

The role of confinement in other geometries - see Sec.6.3.

$$
* * *
$$

In metals positronium formation is not possible, due to positron screening by conduction electrons. However, one observes the positron trapping in defects, where the atoms are missing or their density decreased. To this class of defects belong vacancies, vacancy clusters, dislocations etc. This review is limited to positronium; respective data about positron trapping in metals, ionic crystals and semiconductors one can find in monographs [IV-VI].

\section{ORTHO-PS LIFETIME IN A CLOSED EMPTY VOLUME}

\subsection{The Tao-Eldrup model}

The ortho-Ps trapped in a well annihilates by pick-off process with decay rate $\lambda_{p o}$ or by intrinsic $3 \gamma$ decay $\lambda_{T}$ (Eq. 3). Inside the well the pickoff process does not occur (no electrons are there). The rate of decay by pick-off is a product of the probability $P$ to find a positron outside the well in the electron-rich medium and its decay rate $\lambda_{b}$ in the bulk: 


$$
\lambda_{p o}=\frac{1}{\tau_{p o}}=P \lambda_{b}
$$

The value of $\lambda_{b}$ is not given directly by the theory; one can try to estimate it using the annihilation cross section $\sigma_{2 \gamma}$ for free positron and the electron density of the medium:

$$
\lambda_{b}=\sigma_{2 \gamma} v n Z_{\text {eff }}=4 \pi r_{0}^{2} c n Z_{\text {eff }}
$$

where $r_{0}, c, n$ are, respectively, the classic electron radius, the light velocity in vacuum, the density of molecules. $Z_{\text {eff }}$ is the number of electrons per molecule involved in the annihilation processes, which is not well determined by independent methods ${ }^{6}$. Thus, $Z_{\text {eff }}$ is not strictly the number of electrons, but rather an empirical parameter adjustable for each medium. Another approach, commonly used nowadays, consists in assuming $\lambda_{\mathrm{b}}$ as a constant equal to the spin averaged Ps annihilation rate:

$$
\lambda_{b}=3 / 4 \lambda_{T}+1 / 4 \lambda_{S} \approx 1 / 4 \lambda_{S}=2 \mathrm{~ns}^{-1}
$$

The penetration of positronium into the bulk $P$ depends on the void geometry. The standard version of the discussion assumes the spherical void shape and stepwise radial dependence of the potential. For spherical geometry:

$$
P=4 \pi \int_{R}^{\infty}\left|\psi_{\text {out }}(r)\right|^{2} r^{2} d r
$$

where $R$ is the potential well radius, $\psi_{\text {out }}(r)$ - the radial wavefunction of Ps outside the well. For a particle in the well a whole ladder of energy levels can exist, however, at the size of free volumes appearing in bulk solids and liquids the spacings of energy levels for light particle in the well are much larger than the thermal energy $k T$ at moderate temperatures, thus, even if there are several levels in the well, only the lowest one, $1 s$, is populated . For Ps, treated as a structureless particle, the wavefunction for $1 s$ state outside the well is:

$$
\psi_{\text {out }}(r)=B \exp (-\kappa r) / r
$$

and inside the well:

$$
\psi_{\text {in }}(r)=A \sin (k r) / r
$$

\footnotetext{
${ }^{6}$ One can try to determine $Z_{\text {eff }}$ from the cross section in collisions with single molecules (gas phase), but in this case one obtains usually a very high $Z_{\text {eff }}$, in aromatic molecules it can reach values like $10^{7}$, which do not fit to the case of collisions in condensed matter.
} 
where: $k=\sqrt{4 m_{e} E / \hbar^{2}} ; \quad \kappa=\sqrt{4 m_{e}(U-E) / \hbar^{2}}, U$ is the depth of potential well, $E$ - the energy of level (above the bottom of the well). The coefficients $A$ and $B$ depend on $R, k$ and $\kappa$. The problem of a particle confined in a stepwise (rectangular) potential well is a textbook one; the value of $P$ for a given $R$ can be calculated, e.g. as it is described in [70].

To simplify the calculations Tao [71] proposed to substitute the real potential well of the depth $U$ by an infinitely deep one. In an infinitely deep well the wave-function $\psi_{\infty}(r)$ does not penetrate to outside, thus in Tao's conception the radius of an electron-free void was left equal to $R$, but the radius of the potential well was broadened by $\Delta$ (Fig. 21). The

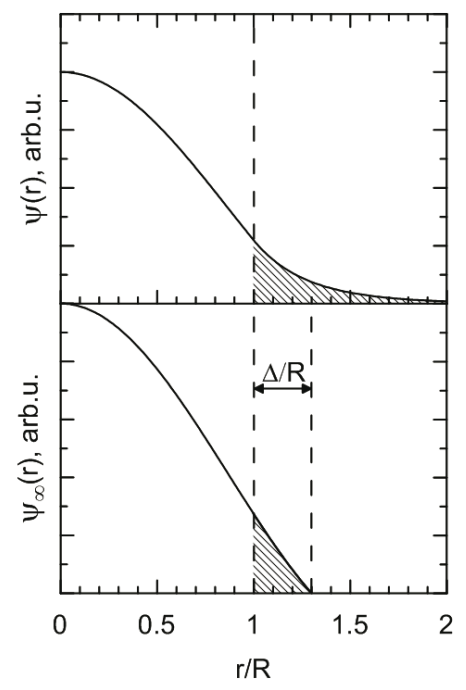

Fig. 21. Radial wavefunction of Ps in a rectangular potential well of radius $R$. Top finite depth; bottom - infinite and broadened by $\Delta$ leaving the radius of an electron-less void unchanged.

value of $\Delta$ is chosen to get the probability $P$ to find Ps inside the electron rich medium the same as for a finite well depth:

$$
\begin{gathered}
P_{\infty}=4 \pi \int_{R}^{R+\Delta}\left|\psi_{\infty}(r)\right|^{2} r^{2} d r=4 \pi \int_{R}^{\infty}\left|\psi_{\text {out }}(r)\right|^{2} r^{2} d r \\
P_{\infty}=\frac{\int_{R}^{R+\Delta} \sin ^{2}\left(\frac{\pi r}{R+\Delta}\right) d r}{\int_{0}^{R+\Delta} \sin ^{2}\left(\frac{\pi r}{R+\Delta}\right) d r}=\left[1-\frac{R}{R+\Delta}+\frac{1}{2 \pi} \sin \frac{2 \pi R}{R+\Delta}\right]
\end{gathered}
$$

The value of $\Delta$ has to be found empirically, if the void radii are known from an independent source, and the values of the o-Ps decay constant come from positron lifetime measurements. 
In a series of papers by Eldrup et al. [72-77] the free volumes accommodating Ps in plastic crystals were identified as vacancies (see Sec. 6.4.1), thus their sizes could be determined from the crystallographic data as the Wigner-Seitz radii of molecules. These radii lie in the range $(0.32 \div 0.38) \mathrm{nm}$. The best agreement between the radii determined from Eqs. 22, 29 and the Wigner-Seitz ones was obtained by Eldrup for $\Delta=0.17 \mathrm{~nm}$. At present the commonly accepted value is $0.166 \mathrm{~nm}$. Fig.22 shows the relation lifetime-void radius according to the TaoEldrup (TE) model.

The $\Delta$ value is fitted to the data from a rather narrow, but typical for polymers, liquids and defected structures, range of radii (respective lifetimes are $2.5 \div 3.2 \mathrm{~ns}$ ). The data for zeolites in Ref.[78] lying in the range $(6 \div 9)$ ns are not fully reliable, due to strong and nonmonotonous lifetime dependence on temperature.

One can compare the dependence $\tau_{\mathrm{o}-\mathrm{Ps}} v s . R$ from the TE model with the calculations for a finite depth of a well, assuming that in the middle of indicated range of radii $(R=0.35 \mathrm{~nm})$ the model and the calculation for finite well depth give the same result. Full agreement for that $R$ is obtained when $U$ is assumed $1.50 \mathrm{eV}[69,79]$. The result of calculation for this value of $U$ is also shown in Fig. 22.

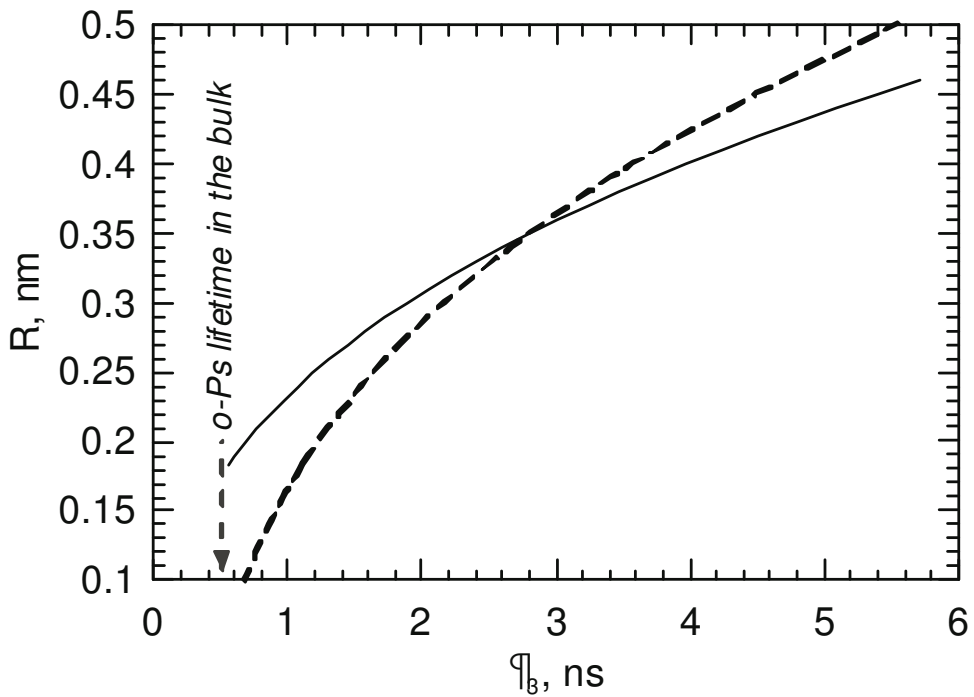

Fig. 22. The relation o-Ps lifetime vs. well radius. Tao-Eldrup model - dashed line, exact solution for rectangular potential well of $U=1.5 \mathrm{eV}-$ solid line [79]. 
The Tao-Eldrup model is a first approximation, so there is the question what an accuracy we need at free volume size determination? If the $10 \%$ difference between the results for an infinite (TE) and more realistic well depth $U=1.5 \mathrm{eV}$ we assume as still acceptable, then one can see in Fig. 22 that the TE model can be used for lifetimes in the range $(1.8 \div 6.2) \mathrm{ns}$. With a shortening of the lifetime the discrepancy of finite and infinite well models rises rapidly, because in the infinitely deep well the energy levels exist at arbitrary small well radius, while it is not possible at finite $U$ value.

The value of the o-Ps decay constant in the bulk $\lambda_{b}$ is commonly assumed as the weighted average of decay rates of para-Ps and ortho-Ps in vacuum, i.e. $2 \mathrm{~ns}^{-1}$ (Eq.24). This approach leaves no place for electron density variation, which is a substantial disadvantage. However, in the majority of cases the formation of positronium is observed in molecular crystals and liquids, polymers, i.e. in organic media, having similar densities, being composed of similar elements - carbon, hydrogen, in much smaller quantity oxygen, nitrogen. For this class of media a universal value of $\lambda_{b}$ is acceptable, but e.g. for silica based materials a modification is probably necessary.

\subsection{Nonspherical geometries}

For elongated voids the approximation of free volume by a cuboid or cylinder is more appropriate. A respective equation for o-Ps confined in a cuboid is $[80,81]$ :

$$
\lambda_{p o}=\lambda_{b}\left[1-\prod_{i=1}^{3}\left(\frac{a_{i}}{a_{i}+2 \Delta}+\frac{1}{\pi} \sin \frac{\pi a_{i}}{a_{i}+2 \Delta}\right)\right]
$$

where $a_{i}$ are the sides of the cuboid, $\Delta$ has the same meaning as for spherical voids. The cuboidal form of the void allows to apply Eq.30 to long channels or to the gaps in a layered structure, assuming one or two dimensions as infinite (for infinite $a_{i}$ the expression in round parantheses is unity). One can extend the Tao-Eldrup model also to the case of an infinitely long cylindrical free volume. For this geometry

$$
\lambda_{p o}=N \lambda_{b} \int_{Z R /(R+\Delta)}^{Z} J_{0}^{2}(r) r d r
$$


where $N$ is a normalization factor: $N=1 / \int_{0}^{Z} J_{0}^{2}(r) r d r=1.28 ; Z=2.41$ is the first zero crossing point of $J_{0}(r)$ Bessel function (cylindrical Bessel function is given in the form of a series only). For the cylindrical channels of finite length one can use the combination of Eqs. 30 and 31. The Eqs. 30-31 contain the $\Delta$ parameter, which needs not to be identical with the one for spherical voids, however, the existing experimental data allow to assume that identity.

Note that the regions of negligible electron density in solids are certainly not spherical, the potential is not stepwise, moreover, free volumes need not form separate cavities, being sometimes rather an irregular three-dimensional tangle between the molecules. The o-Ps lifetime gives often to us the radius of an equivalent sphere only.

\subsection{Two-particle structure of $P S$}

In the discussion about the relation between the size of free volum and o-Ps lifetime, presented above, positronium was assumed to be a structureless particle. Attempts to account for the Ps structure were undertaken by Larrimore et al. [82, 83]. They have performed Monte Carlo calculations for two-particle electron-positron system confined within a hard sphere (i.e. $U=\infty$ ). In Fig. 23 the radial density distribution of $\mathrm{e}^{+}$bound in the Ps structure (dots) calculated by these authors for a sphere radius 6 a.u. $(0.265 \mathrm{~nm})$ is shown; asterisks represent that density for a structureless Ps particle. The difference between the two variants is well visible.

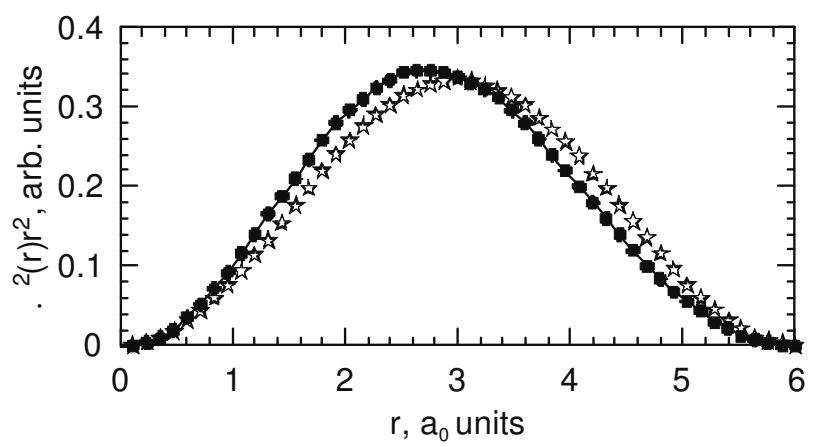

Fig. 23. Radial distribution of positron density. Full symbols: positron in twoparticle structure of Ps, asterisks: positron in structureless Ps. Well radius 6 a.u. (After Larrimore et al. [82]). 
Larrimore et al. compared the $P$ values calculated as in Tao-Eldrup model and for an $\mathrm{e}^{+}-\mathrm{e}^{-}$system assuming a rather realistic $\Delta=3$ a.u. $(0.159$ $\mathrm{nm}$, i.e. close to the commonly used $0.166 \mathrm{~nm})$. In the range $R=(5 \div 10)$ a.u. the values differed from those of the TE model by about 10-20\% However, as long as the Tao concept of an infinite well and penetration parameter $\Delta$ are used, there is no essential difference between the TaoEldrup model and the refined Larrimore approach: substituting in Eq. 29 the $\sin r / r$ wavefunctions by the distributions calculated by these authors one has to modify respectively the $\Delta$ value to get the same $P$ value as in the simple TE model ( $\Delta$ is an empirical parameter only).

$$
* * *
$$

In this review we put aside the whole positronium chemistry, which for liquid media is a rich field of research.

\section{4, Positronium in a magnetic field}

Both, para- and ortho-Ps states have no magnetic moment, however, in the magnetic field the second-order Zeeman effect is observed; it follows from mixing the $m=0$ substates ortho and para [84]. The ortho substates with $m= \pm 1$ are not mixed, insensitive to the magnetic field. The o-Ps part in a PAL spectrum consists now of two components: one quenchable by the field $\tau_{3}$, the other - insensitive, $\tau_{3}$. The admixture of singlet state shortens the lifetime of the triplet one (admixture of triplet to the singlet gives a negligible effect, due to the very low value of $\lambda_{T}$ ):

$$
\lambda_{3}=\frac{y^{2} \lambda_{S}+\lambda_{3}}{1+y^{2}}
$$

where $y=\left(\sqrt{1+x^{2}}-1\right) / \mathrm{x}$ is the mixing factor, $x=4 \mu_{B} B / \Delta W ; \mu_{B}, B, \Delta W$ denote the Bohr magneton, magnetic induction and energy distance of unperturbed para and ortho states, respectively.

The effect of magnetic field can be easily observed by comparing the area under the fragment of PALS spectrum between two points selected on the tail of lifetime distribution, $t_{a}$ and $t_{b}$ (Fig.24) when the sample is in magnetic field and out of the field [85]. The ratio of areas $R$ is:

$$
R=\frac{2}{3}+\frac{1}{3} \frac{\exp \left(-\lambda_{3} t_{a}\right)-\exp \left(-\lambda_{3} t_{b}\right)}{\exp \left(\lambda_{3} t_{a}\right)-\exp \left(-\lambda_{3} t_{b}\right)}
$$




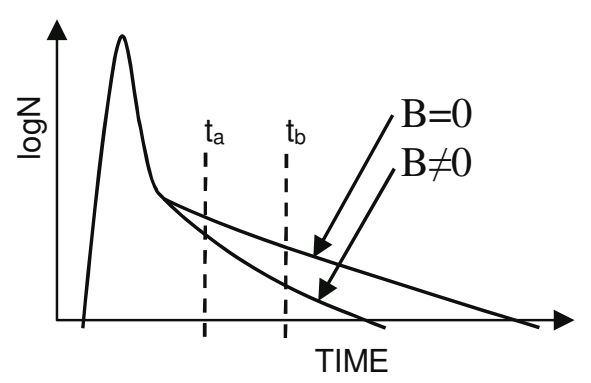

Fig. 24. The $R$ parameter is the ratio of areas in the PAL spectrum between the points $t_{a}$ and $t_{b}$ in the presence of a magnetic field and without that field.

The term $2 / 3$ in the equation above relates to the $m= \pm 1$ components. The points $t_{a}$ and $t_{b}$ have to be chosen at sufficiently large delay to be sure that the tail of spectrum does not contain other components but o-Ps. An example of $R$ as a function of magnetic field induction is shown in Fig. 25 [86].

The hyperfine splitting of positronium ground state $\Delta W$ in vacuum is:

$$
\Delta W=\frac{14 \pi \alpha \hbar^{3}}{3 m_{e}^{2} c}|\psi(0)|^{2}
$$

but in a medium it can be modified due to the change of electron density at the positron's site $|\psi(0)|^{2}$ (contact electron density). We denote the ratio of electron density in positron's site in the medium under study to that density for Ps in vacuum $\zeta$ :

$$
\zeta=\frac{\left|\psi(0)_{\text {med }}\right|^{2}}{\left|\psi(0)_{\text {vac }}\right|^{2}}
$$

and then in Eq.32 the value of $\Delta W$ has to be substituted by $\zeta \Delta W$. This modifies the mixing factor, e.g. if $\zeta<1$, the mixing factor increases and the $R$ dependence on the field $B$ is compressed along $B$ axis. Such a case is shown in Fig. 25 [86], the best fit of the theoretical curve to the experimental data is for $\zeta=0.8$.

The parameter $\zeta$ rules also the lifetime of para-positronium:

$$
\lambda_{S}=\zeta\left(\lambda_{S}\right)_{v a c}=4 \pi r_{0}^{2} c \zeta|\psi(0)|^{2}
$$

(generally, this $\zeta$ need not to be identical with $\zeta$ from Eq.35, but a possible deviation is small [87]). In the majority of cases $\zeta$ is found less than unity, it means one should observe lengthening of the para-Ps lifetime compared to its vacuum value. In the ortho-Ps decay this effect is not visible, being shadowed by the dominant pick-off process. 


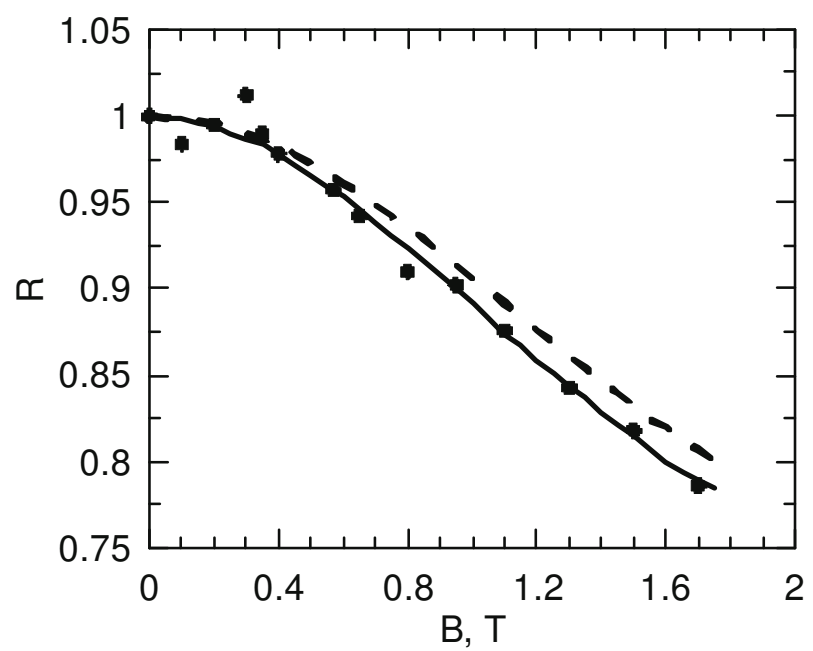

Fig. 25. $R$ parameter as a function of magnetic field induction in p-terphenyl doped with anthracene (single crystal). The continuous line is $R$ expected for $\zeta=0.80$, dashed line is for $\zeta=1$ [86]. The lifetime of the $\tau_{3}$, component decreases from $1.44 \mathrm{~ns}$ without magnetic field, to $0.89 \mathrm{~ns}$ at $B=1.7 \mathrm{~T}$ (quenchable ortho-Ps substate with admixture of triplet is called meikto-Ps),

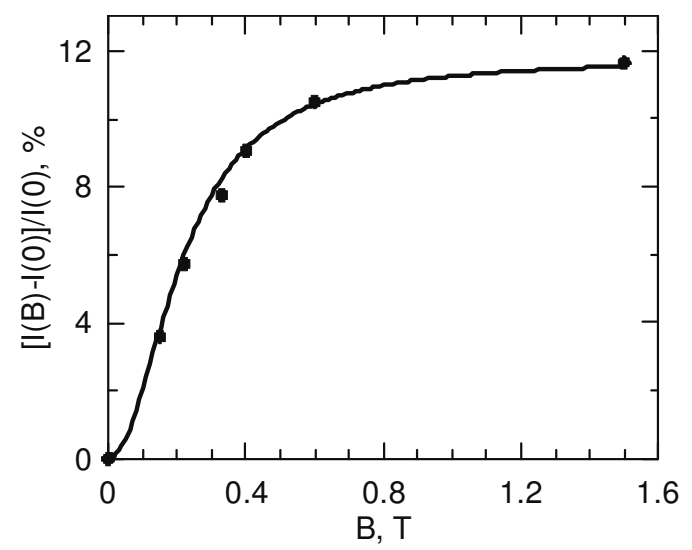

Fig. 26. Relative increase of the $511 \mathrm{keV}$ intensity as a function of magnetic field $B$ for porous zeosil $(\zeta=1)[88]$. The solid line represents the equation (37).

The admixture of para- to ortho- state induces the two-quantum decays of that state and thus an increase of the intensity of the $511 \mathrm{keV}$ peak in the $\gamma$ energy spectrum. The intensity of $511 \mathrm{keV}$ decays in a magnetic field $I_{511}(B)$ relative to that intensity without field $I_{511}(0)$ is:

$$
\frac{I_{511}(B)-I_{511}(0)}{I_{511}(0)}=\frac{I_{3}}{\left(1-I_{3}\right)} \frac{y^{2} \lambda_{S}}{3\left(y^{2} \lambda_{S}+\lambda_{3}\right)}
$$


where $I_{3}$ is the intensity of o-Ps component. Fig. 26 shows the increase of total area under the $511 \mathrm{keV}$ peak with the magnetic induction $B$ for porous zeosil [88]. In this medium the intensity of the longest-lived component is $30 \%$ and the lifetime $140 \mathrm{~ns}$, thus the share of $3 \gamma$ decays is particularly high.

\subsection{Positronium in an electric field}

The probability of Ps formation depends on the electric field in the sample. In the simple version of spur model [55] the Eq.16 is modified to:

$$
P=1-\left(1+\frac{e D r_{c}}{2 k T}\right) \exp \left(-\frac{r_{c}}{d}\right)
$$

where $D$ is the electric displacement field. The theory for a more realistic case of Ps formation in the blob containing many ionization products is given by Stepanov and Byakov [89]. An electric field can reduce the Ps intensity, down to about half of that observed without the field. The electric field effect was observed by Brandt and Wilkenfeld [90] and some other authors. Examples of the influence of an external electric field are shown in Fig. 27 [91,92]. In phenanthrene in the range to about $30 \mathrm{kV} / \mathrm{cm}$ the decrease of $I_{3}$ intensity is almost linear (Fig. 27), $d I_{3} / d E$ is about $2.9 \mathrm{~cm} / \mathrm{MV}$, similar to that reported by Brandt and Wilkenfeld [90].

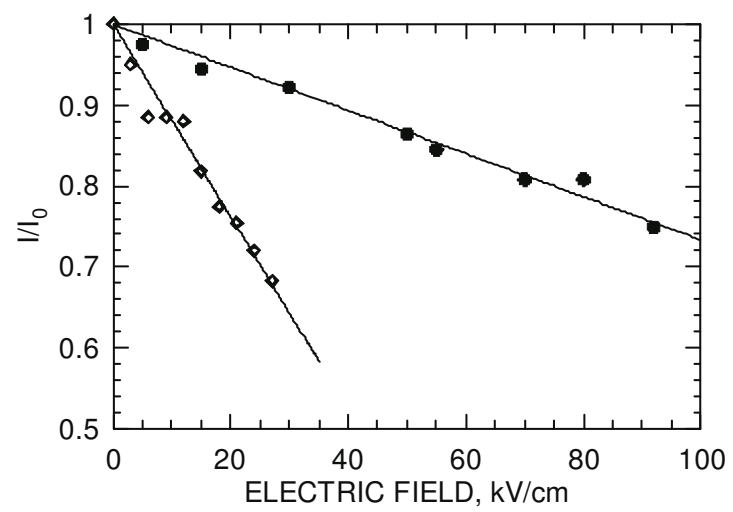

Fig. 27. Relative o-Ps intensity in the electric field. Dots - teflon (polymer), open diamonds - phehanthrene (crystal). $I_{0}$ means the o-Ps intensity without electric field. Compiled of the data from Ref.91 and 92. 


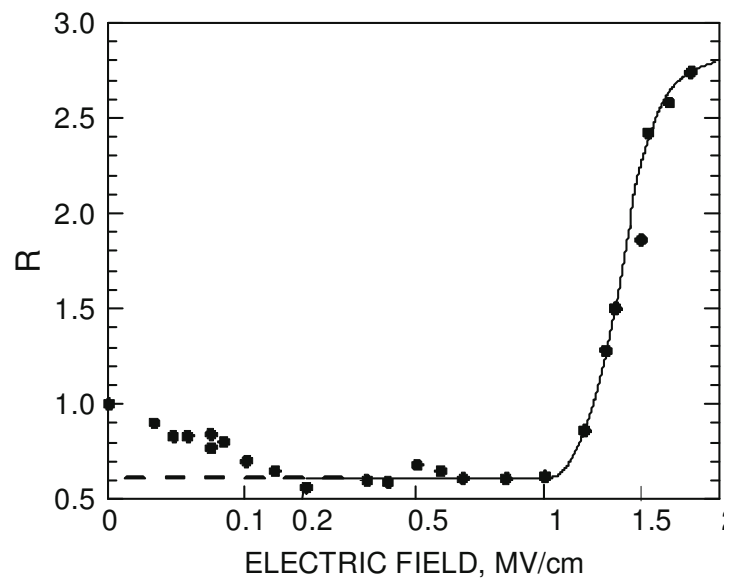

Fig. 28. The $R$ parameter (defined as in the case of magnetic field) as a function of electric field strength $E$ in polypropylene (according to [93]). The abscissa axis is drawn as a square root of electric field.

At very strong electric fields one observes a reversed effect (Fig. 28) - the intensity of longest-lived component rises to the values higher than without field [93]. According to Bisi et al. [93] this process is ruled by the Ore mechanism. A strong electric field can accelerate the slowed down positrons back to Ore gap making possible the ionization of atoms with simultaneous Ps formation.

The sensitivity of positronium formation to the electric field acting in the sample allows to use positron methods to investigate the fields generated during irradiation, or e.g. to observe the pyroelectric effect. This effect consists in the generation of an electric field in a dielectric at the change of temperature. As an example of pyroelectric medium one can give solid phenanthrene. At $345 \mathrm{~K}$ an order-disorder phase transition appears (in this case disorder consists in two possible molecular positions). Above this point there is no pyroelectric effect, while below it one observes the sample polarization at the change of temperature. Polarization of the sample is

$$
P=\int_{T_{1}}^{T_{2}} p(T) d T
$$

where $T_{1}$ and $T_{2}$ are initial and final temperatures, $p(T)$ is the pyroelectric coefficient. In phenanthrene this coefficient is peaked just below the phase transition point and comes to zero at $30^{\circ} \mathrm{C}-40^{\circ} \mathrm{C}$; electric field generated in this solid can reduce the o-Ps intensity by $50 \%$ [92]. 
The lifetime of o-Ps is almost insensitive to the presence of sample polarization, the reduction of lifetime, if any, does not exceed 5\%.

\section{POSITRONIUM IN MOLECULAR CRYSTALS}

From the viewpoint of positronium formation one can distinguish three groups of molecular crystals, in which:

- no positronium within the whole range of solid phases (in liquid one Ps can exist),

- positronium can be formed if the crystal structure is perturbed,

- positronium is formed at any temperature in natural free spaces existing in the structure.

As a representative of the first group can serve anthracene. Its PALS spectrum contains one exponential component only up to $5 \mathrm{~K}$ below the melting point $490 \mathrm{~K}$ [94]. In the range of $5 \mathrm{~K}$ below the melting point long-lived components begin to appear due to growing number of defects and finally in the liquid phase the o-Ps component reaches the intensity of about $30 \%$.

\subsection{Doped organic crystals}

In many tightly packed crystalline structures positronium does not form - no empty regions exceeding in size $R_{\min }$ given by Eq. 18 . However, perturbations of the structure can increase the small free volumes to the extent sufficient for positronium accommodation. As an example of such a perturbation can serve the introduction of foreign molecules (guests) crystallizing together with the main component (host). When the guest molecules are smaller in size than the host, there is an enlarged free space in their neighbourhood, larger than that in the neat crystal. For example, in neat $p$-terphenyl at room temperature positronium does not form; in the PALS spectrum one observes a single exponential component only (annihilation of free positrons) and the momentum distribution does not contain the narrow component. However, the admixture (less than one percent) of three- or four-ring polynuclear aromatic molecules is enough to cause the appearance of both mentioned components [95, 96, 97]. Fig. 29 shows the dependence of the long-lived component in PALS and narrow component in ACAR as a function of admixture concentration (3-ring anthracene or 4-ring benzofluorene). 


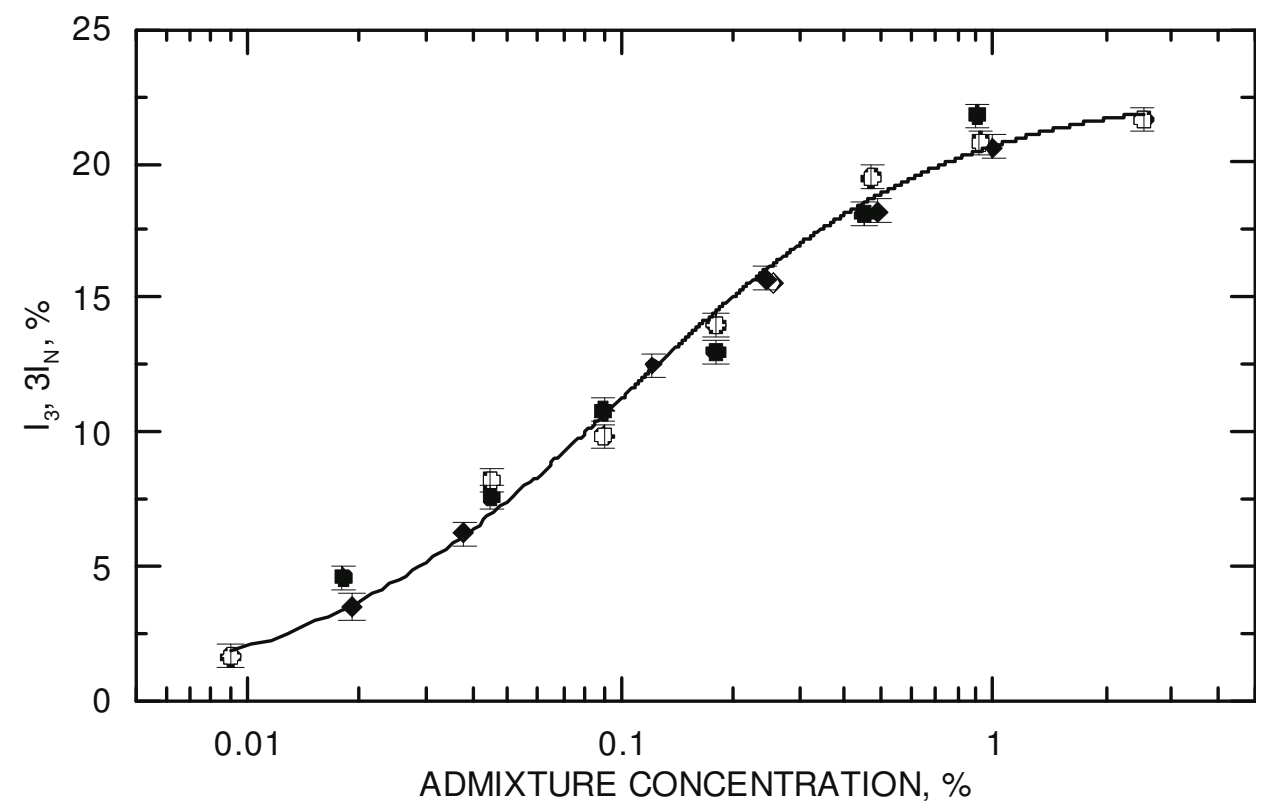

Fig. 29. Intensities of the para- and ortho-positronium as a function of the guest molecule concentration in p-terphenyl host. Guests: diamonds benzofluorene, circles - anthracene; full symbols are for long-lived component, open symbols - for a narrow component in ACAR $(\times 3)$. From [97].

If we accept the mechanism of Ps formation proposed by Stepanov (see Sec. 4), and denote $P_{p}$ the probability of formation of the correlated $\mathrm{e}^{+} \mathrm{e}^{-}$pair (quasi-free positronium, qPs) after the blob collapse and $K-$ the trapping rate of $\mathrm{qPs}$ to the voids, we receive the form of spectrum as below:

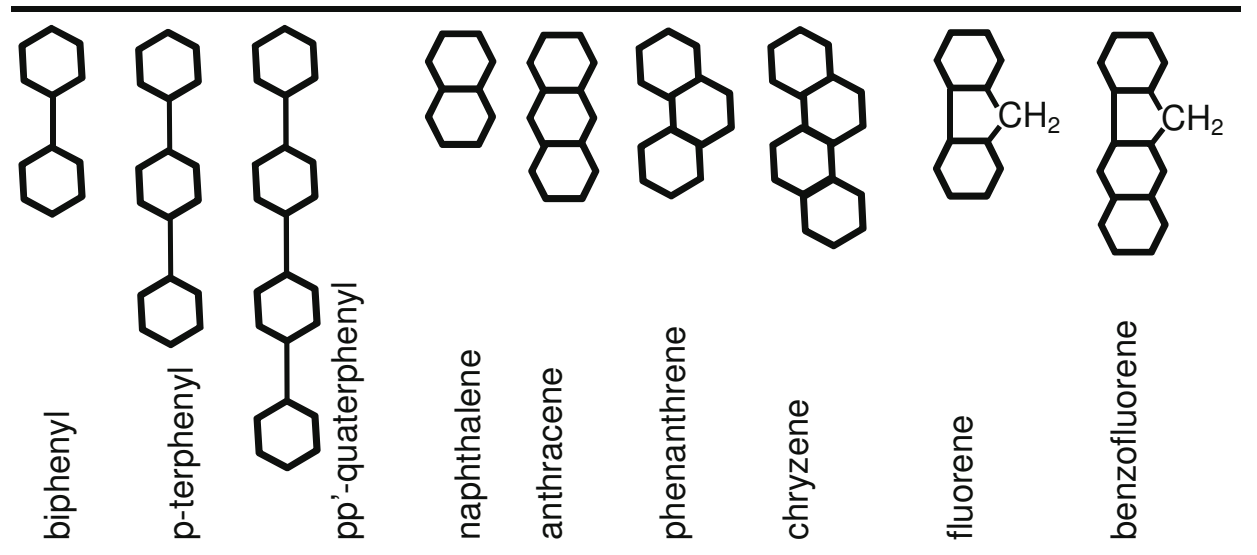




$$
\begin{aligned}
& f(t)=\frac{1}{4} \frac{P_{p} \lambda_{1} K}{\lambda_{2}-\lambda_{3}+K} \exp \left(-\lambda_{1} t\right)+\left(1-P_{p}\right) \lambda_{2} \exp \left(-\lambda_{2} t\right)+ \\
& P_{p}\left(\lambda_{2}-\frac{\lambda_{3} K}{\lambda_{2}-\lambda_{3}+K}\right) \exp \left[-\left(\lambda_{2}+K\right) t\right]+\frac{3}{4} P_{p} \frac{\lambda_{3} K}{\lambda_{2}-\lambda_{3}+K} \exp \left(-\lambda_{3} t\right)
\end{aligned}
$$

The terms 1, 2, 4 look like typical exponential decays of para-Ps, free annihilation, ortho-Ps, respectively. An additional third term describes qPs annihilation before binding into Ps trapped in a well. The average $\mathrm{e}^{+}-\mathrm{e}^{-}$distance in $\mathrm{qPs}$ is of the order of $0.5 \mathrm{~nm}$ [58], therefore the positron has in its vicinity plenty of electrons located closer than the electron belonging to the pair and it annihilates mainly with them, like in the case of free $\mathrm{e}^{+}$annihilation. This term for $K<<\lambda_{2}$ is almost identical with the free annihilation component, for $\mathrm{K} \rightarrow \infty$ the lifetime shortens, but the relative intensity tends to 0 . Due to these properties the qPs annihilation term is difficult to observe experimentally and there is no firm proof of its appearance. According to Eq. 40 the long-lived or narrow component intensities are:

$$
I_{3, N}=I_{\max } \frac{K}{\lambda_{2}-\lambda_{3}+K}
$$

where $I_{\max }$ is $3 / 4 P_{p}$ for the long-lived component and $1 / 4 P_{p}$ for the narrow one. For moderate concentration of guest molecules the trapping rate $K$ is proportional to that concentration, $K=\mu c$. The solid curve in Fig. 29 represents the function from Eq. 41 fitted to the experimental data. Note that the impurity induced long-lived component in p-terphenyl becomes visible already at an admixture concentration of the order of $10^{-4}$ (in the case of hydrogen fluoride HF in ice this limit can be shifted further down, to $10^{-6}$ [98]). The reciprocal of $\mu$ can be assumed as the formation time if free volume is available everywhere $(c=1)$, like in liquids. From Fig. 29 one obtains that time $\approx 1 \mathrm{ps}$, thus, consistent with the radiation chemistry data.

In p-terphenyl with an anthracene admixture the guest molecule can be located in 16 nonequivalent positions in the crystal cell [99], and essentially one could expect 16 different long-lived components. The experiment shows one component $\left(I_{3}, \lambda_{3}\right)$ only, thus respective lifetimes must be very close to each other and we observe an average of them. One can estimate qualitatively the relation between the void size and the parameters characterising the annihilation process. The molecules of p-terphenyl are elongated and the rule "shorter guest - longer o-Ps lifetime" seems to be well proven. 


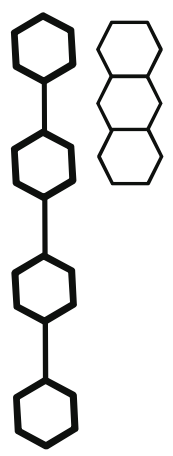

1,67 ns

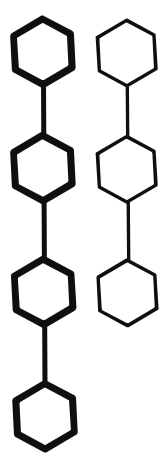

1,48 ns

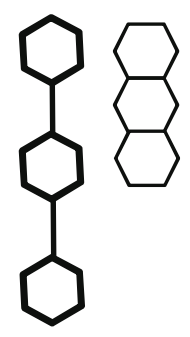

1,44 ns
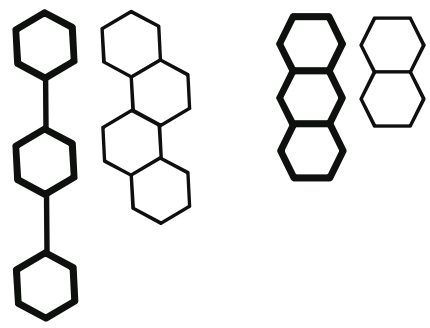

1,14 ns

Fig. 30. Ortho-Ps lifetimes for various host-guest pairs. Thick line - host, thin line - guest.

Fig. 30 shows the host-guest pairs with respective lifetime values. For example, if the guest in p-terphenyl lattice is a three-ring polynuclear molecule, the o-Ps lifetime $\tau_{3}$ is about $1.4 \mathrm{~ns}$, while if it is four-ringed one - about $1.1 \mathrm{~ns}$. The size of free volume determines the o-Ps lifetime and also the width of the p-Ps component in an ACAR spectrum; longer lifetime - narrower p-Ps component. This correlation is visible in Fig. 31.

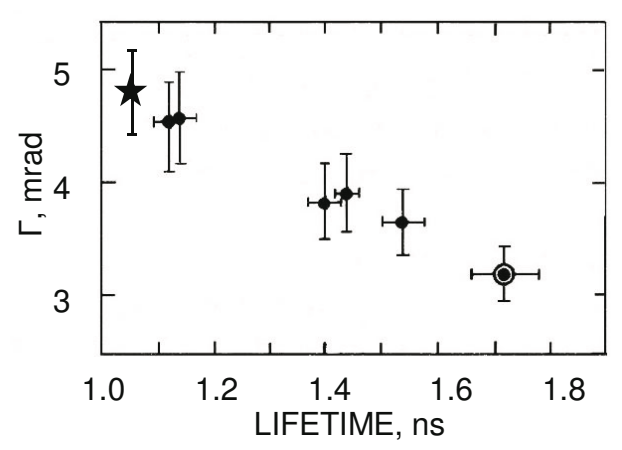

Fig. 31. Halfwidth of the narrow (p-Ps) component in ACAR vs. orthoPs lifetime. Asterisk - anthracene $+1 \%$ naphthalene. Dots: host p-terphenyl, guests from left to right: benzofluorene, crysene, fluorene, anthracene, carbazole; double dot acenaphthylene polymer [97].

\subsection{Temperature and pressure effects}

In some cases there is no positronium in pure crystals at low temperatures, but by increasing the temperature we can get small free volumes extended to the size that enables Ps accommodation. A classic example can be the naphthalene crystal. Naphthalene was probably the first organic solid in which Ps was observed and the temperature dependence of its formation investigated [100,101]. A stepwise change of the Ps yield was observed at the melting point, but between melting and 
room temperature no changes were seen. However, with further lowering of the temperature the intensity of the long-lived component diminished (Fig. 32) reaching zero at $\approx 220 \mathrm{~K}[102]$.

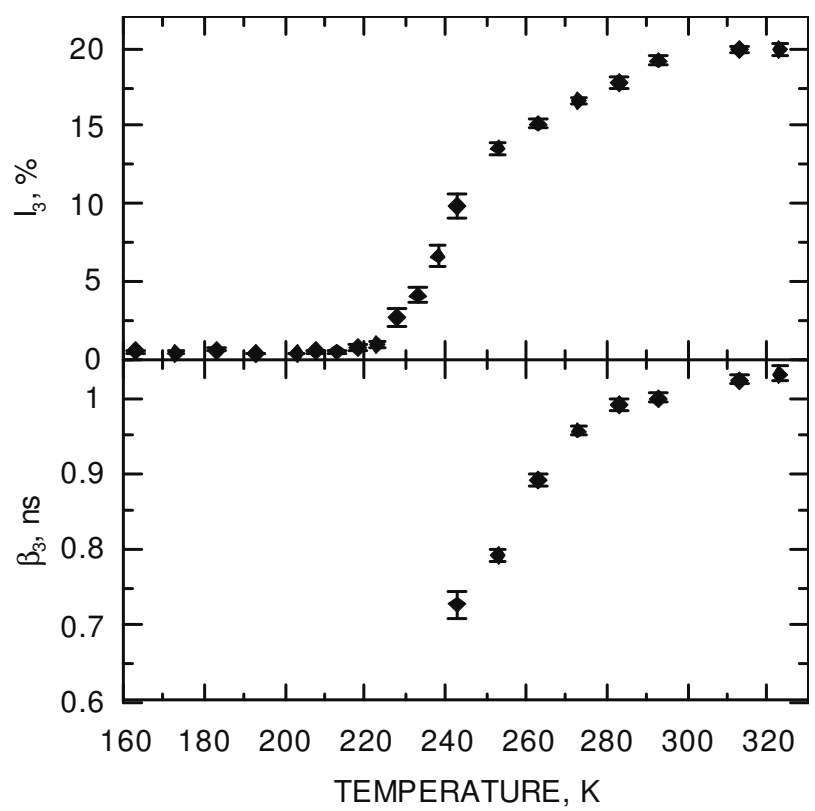

Fig. 32. Temperature dependence of the o-Ps intensity and lifetime in naphthalene [102]. The values of $\tau_{3}$ below $230 \mathrm{~K}$ are not shown due to large uncertainity and scatter of values when $I_{3}$ is below $1 \%$.

The sigmoidal dependence of the intensity $I_{3}$ on temperature suggests a thermal activation of Ps accommodating defects, then their concentration should be:

$$
c \propto \exp \left(-H_{v} / k T\right)
$$

where $H_{v}=E_{v}+p V_{v}$ is the activation enthalpy of defects (the term $p V$ can be usually neglected). The slope of Arrhenius plot: $\ln \left[I_{3} /\left(I_{3 \max }-I_{3}\right)\right] v s .1 / T$ allows us to determine the enthalpy, which for naphthalene is found to be $H_{v}=0.2 \mathrm{eV}$ (Fig. 33).

One could suppose that in this case vacancies serve as the centres of Ps formation and their concentration corresponds to the equilibrium state. However, for vacancies the formation enthalpy should be equal to the sublimation heat $L_{s}$ which is $0.7 \mathrm{eV}$. Moreover, the molecular volume of naphthalene is about $0.173 \mathrm{~nm}^{3}$ [103] and for such a volume the TaoEldrup model (and its modified version with finite $U$ ) predicts the lifetime of $2.7 \mathrm{~ns}$, while in the experiment the observed lifetime is close to $1 \mathrm{~ns}$. 


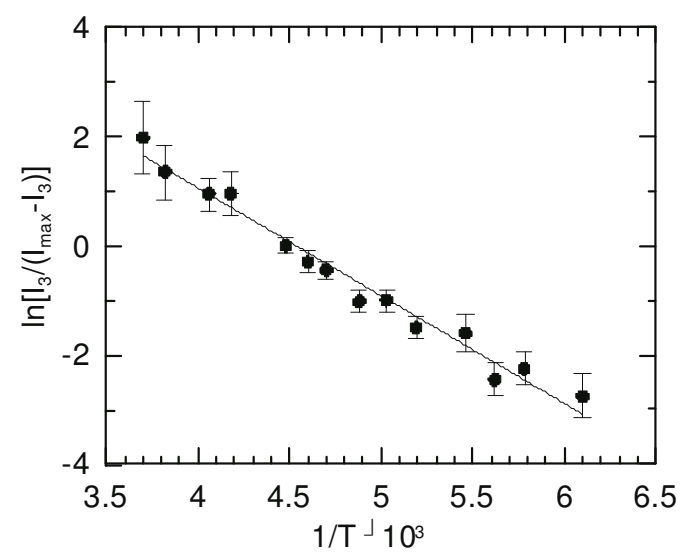

Fig. 33. Arrhenius plot for naphthalene (R. Wasiewicz, Ph.D. Thesis, UMCS Lublin 1985).

Thus, in naphthalene the free volumes accommodating Ps cannot be vacancies. It seems that Ps is hosted in free volumes formed by intermolecular spaces. The thermal motion of molecules or even of single atoms in the molecule induces a variation of these volumes and this locally increased volume can be sufficient to trap Ps. This supposition is confirmed by X-ray studies [103]; mapping the electron density (atom localization) in the crystal cell of naphthalene becomes impossible above $240 \mathrm{~K}$ due to excessive molecular motion. The free volumes rise also owing to thermal expansion of the crystal structure. A similar temperature dependence of the Ps yield was seen in biphenyl [104] and chrysene [105]. In both cases the activation energy of Ps traps was $\approx 0.2 \mathrm{eV}$.

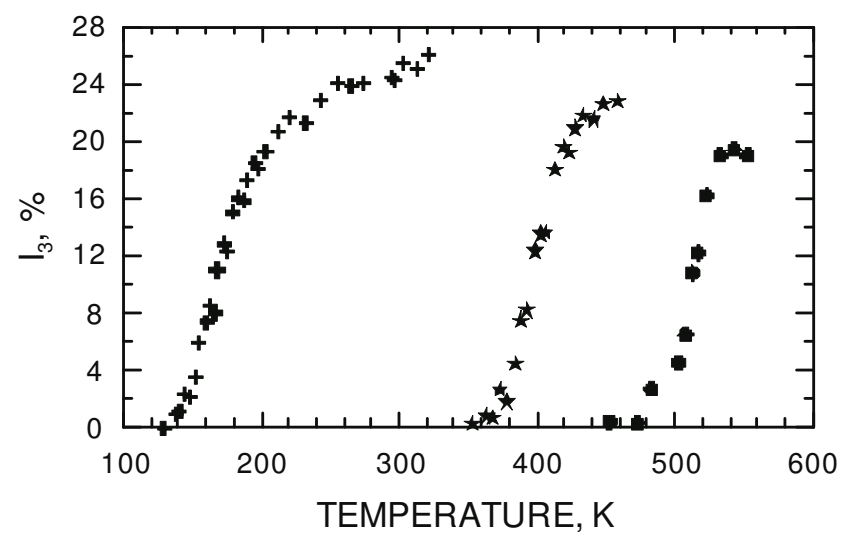

Fig. 34. Temperature dependence of the o-Ps intensity in biphenyl (crosses), p-terphenyl (asterisks) and pp'-quaterphenyl (dots) [104].

The same effect appeared also in other polyphenyls, like p-terphenyl or pp'-quaterphenyl (Fig. 34), but at much higher temperatures [104]. 
An Arrhenius plot for quaterphenyl gives an unrealistic value of the activation enthalpy of $2.4 \mathrm{eV}$.

If the centres of positronium trapping are intermolecular free spaces, they have to exist at low temperatures too, but their sizes lie below the minimal radius (equivalent radius) $R_{\min }^{\prime}$. With the increase of temperature they expand and if they have a certain distribution of sizes the number of them exceeding the threshold $R$ value increases too. In this case the concentration $c$ in Eq. 42 relates only to these free volumes which found themselves above the threshold and the concentration need not to be described by an exponential of $H_{v} / T$. In such a case the larger value of $d I_{3} / d T$ would be not the effect of higher $H_{v}$, but rather of narrower distribution of free volume sizes. If this supposition is true the concentration $c(T)$ would be described rather by an $\operatorname{erf}\left(T-T^{*}\right)$ function, not an exponential.

At a depth of the potential well equal to $1.5 \mathrm{eV}$ (as it is needed to justify the $\Delta$ value in the Tao-Eldrup model), the $R_{\min }^{\prime}$ is $0.177 \mathrm{~nm}$. In p-terphenyl the o-Ps component begins to appear at about $370 \mathrm{~K}$.

An extrapolation of the set of $R$ values (Fig. 35), calculated for finite well depth (see solid line in Fig. 22) from the experimental lifetime values down to $370 \mathrm{~K}$, gives $R_{\text {min }}^{\prime}=0.186 \mathrm{~nm}$, not far from that expected for $U=1.5 \mathrm{eV}$.

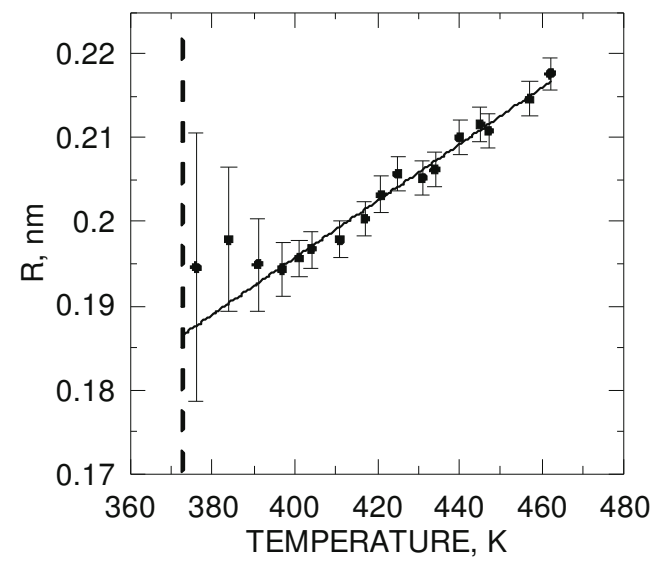

Fig. 35. Radius of free volumes active in Ps formation in p-terphenyl as a function of temperature. Radius $R$ was calculated assuming finite potential well depth $U=1.5 \mathrm{eV}$. Vertical dashed line indicates the threshold of Ps appearance in p-terphenyl.

If positronium is located in small thermally expanding volumes (like in biphenyl or naphthalene) one can expect that under pressure their sizes decrease, the energy of the Ps level in the well shifts up, and according to Eq.18 at certain $R_{\min }$ the level disappears; Ps trapping becomes impossible and the PALS spectrum does not contain the long- 
lived component any more. An experiment of this kind was done e.g. for biphenyl [106] and naphthalene [107]; one of these results is shown in Fig.36.

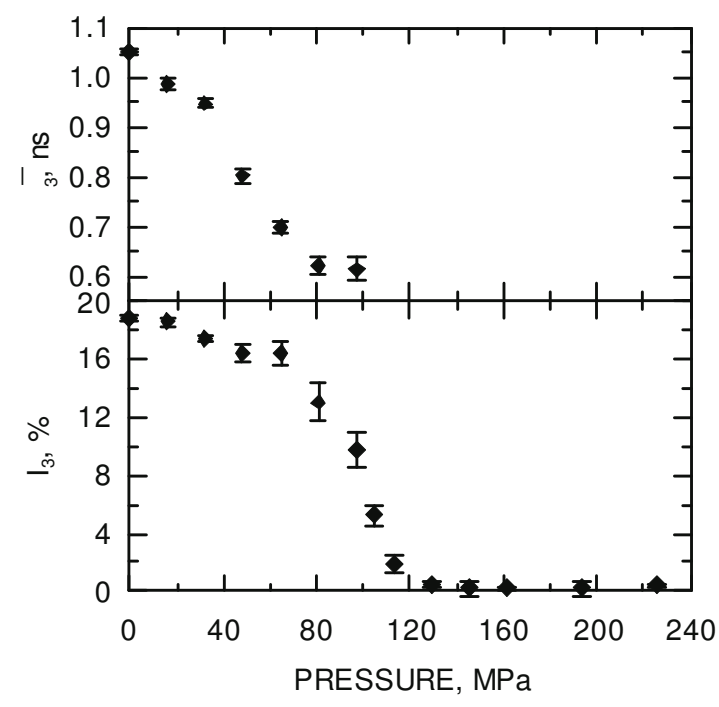

Fig. 36. Pressure dependence of the o-Ps intensity and lifetime in naphthalene, at room temperature [107].

\subsection{Positronium in lamellar structures}

As representatives of molecular solids in which the positronium is formed at arbitrary temperature, can serve alkanes. The normal alkanes (n-alkanes) are saturated hydrocarbons $\mathrm{C}_{n} \mathrm{H}_{2 n+2}$ (for short $-\mathrm{Cn}$ ) in the form of carbon chains. Their molecules can appear in various geometric forms (conformers). At low temperatures they have the form of straight zig-zag chain (all-trans arrangement of $\mathrm{C}-\mathrm{C}$ bonds), at higher temperatures the concentration of non-planar conformers rises: one or two last bonds are turned out the $\mathrm{C}-\mathrm{C}$ zigzag plane (end-gauche and doublegauche conformers), two linear fragments linked by gauche-trans-gauche fragment (kink conformer) etc.(Fig. 37).

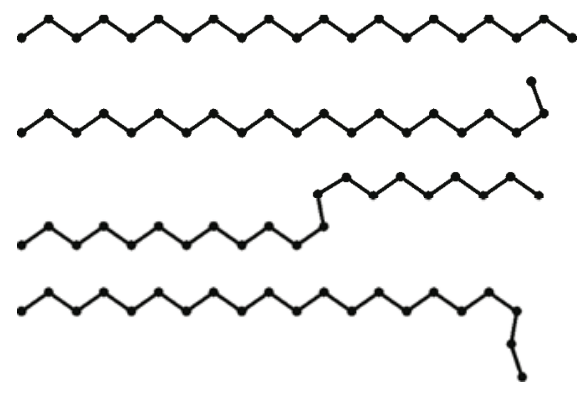

Fig. 37. Conformers of n-alkanes, from top to bottom: all-trans, end-gauche, kink, double gauche. Hydrogen atoms are not shown. 
In the liquid (and gaseous) phase the carbon chains are flexible, the molecules can appear in various, curly forms, containing a great number of gauche bonds. Alkane crystals form a lamellar structure; within the lamella the molecules are packed in parallel and the spacing between lamella layers is $0.125 \mathrm{~nm}$ in even-numbered, $0.195 \mathrm{~nm}$ in odd-numbered [108] molecules. In solid even numbered long-chain alkanes the o-Ps lifetime rises slowly with temperature (Fig. 38).

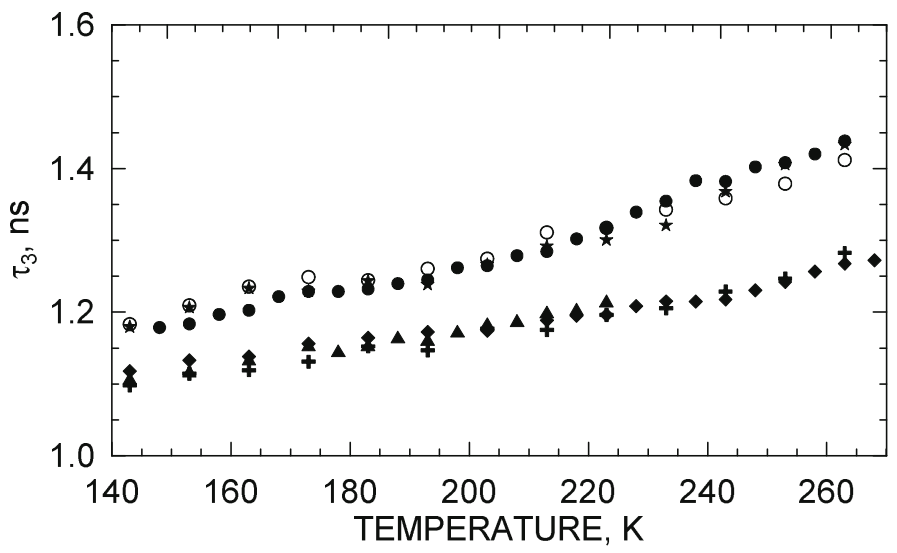

Fig. 38. Temperature dependence of the o-Ps lifetime in rigid phase of n-alkanes: C16 (triangles), C18 (crosses), C19 (dots), C20 (diamonds), C21 (empty circles), C23 (asterisks).

The o-Ps lifetime values for odd-numbered chains lie along an analogous curve, but shifted upwards (about $150 \mathrm{ps}$ ) relatively to the curve for even-numbered ones [109]. The difference of lifetimes between these two groups of alkanes is an indication that Ps is trapped in the interlamellar spaces. Inside the lamella the molecules are too tightly packed to leave sufficient free space to accommodate the Ps atom. An additional argument in favour of such Ps location is an increase of the oPs lifetime in mixed alkanes. X-ray investigations show that with adding an admixture of alkane with different chain length (shorter or longer than the chain of main component) the width of the interlamellar gap increases. Fig. 39 presents the temperature dependence of the lifetime for neat n-eicosane $\mathrm{C} 20$ and with an admixture of n-octadecane C18. At the octadecane content $4 \%$ the lifetime increases to the values like in odd numbered alkane, the the admixture of $10 \%$ gives a further extension of the lifetime. 


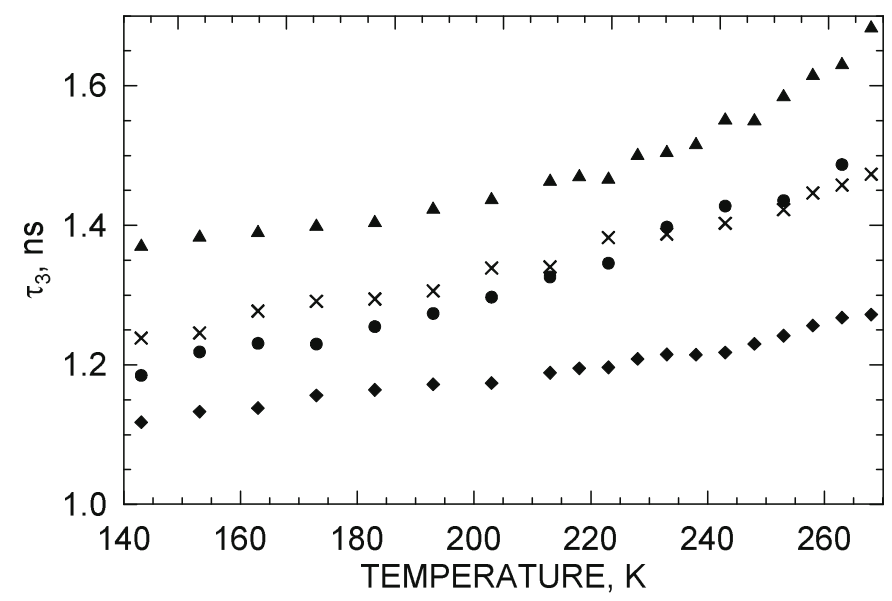

Fig. 39. Temperature dependence of the o-Ps lifetime $\tau_{3}$ in: pure $\mathrm{C} 20$ (diamonds), pure C19 (dots), $\mathrm{C} 20+4 \% \mathrm{C} 18$ (crosses), $\mathrm{C} 20+10 \% \mathrm{C} 18$ (triangles).

As a next example supporting the concept of Ps location in the interlamellar gap can serve the lifetime spectrum of n-hexatriacontane C36. The X-ray scattering measurements for this alkane near room temperature prove the coexistence of two solid phases [110]. The PALS measurements at 303 and $313 \mathrm{~K}$ analysed by the MELT routine (Fig.40) show two o-Ps peaks, thus belonging to two phases [111].

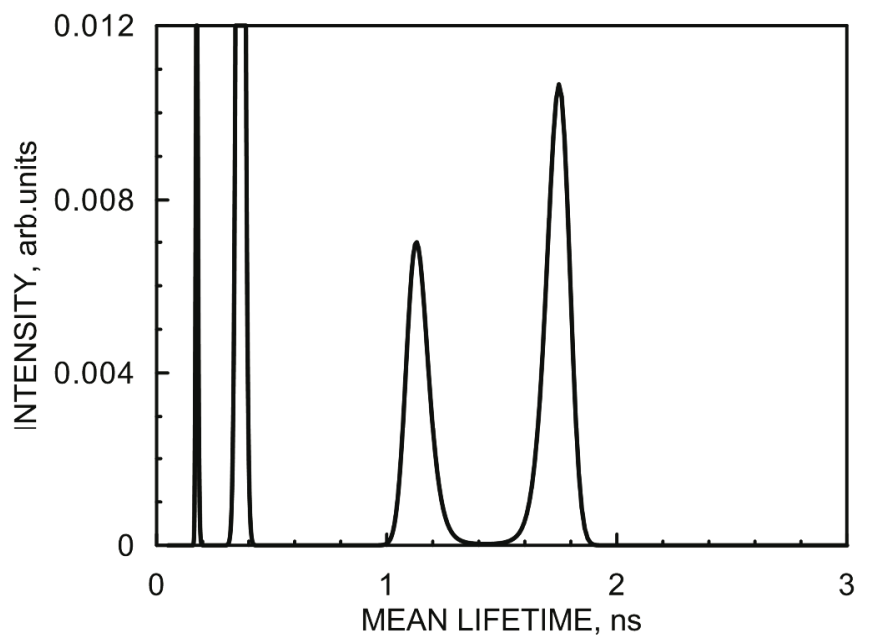

Fig. 40. Distribution of mean lifetimes in n-hexatriacontane C36 measured at 303 K. Peaks from left to right: para-Ps, free annihilating $\mathrm{e}^{+}$, two orthoPs peaks of two coexisting phases (MELT program analysis [111]). 
The peak at about $1.2 \mathrm{~ns}$ is typical for low-temperature rigid phase, the other one, located at $1.8 \mathrm{~ns}$, can belong to the other phase. The X-ray measurements show that the interlamellar gap in that phase is $0.36 \mathrm{~nm}$, more than twice of that in ordinary shorter chain even alkanes, which explains such a large lifetime value.

The structure of solid long-chain aliphatic alcohols $\mathrm{C}_{n} \mathrm{H}_{2 n+1} \mathrm{OH}$ looks similar to that of alkanes: the long molecules are arranged in lamellae. The o-Ps lifetime rises slowly with temperature, $\mathrm{dI}_{3} / \mathrm{dT}$ is about $1.5 \mathrm{ps} / \mathrm{K}$, reaching about $1.4 \mathrm{~ns}$ near the melting point.

If Ps in alkanes locates in the interlamellar gap, its accommodation place has the peculiar shape of a slit in the otherwise tight structure. In a perfect crystal two dimensions of such a void would extend to infinity, and even if the real structure is disturbed by defects, only one dimension - namely the gap width - forms a strong confinement. The calculations described in Sec. 4.2 give the lower limit for Ps existence in threedimensional (spherical) confinement, $2 R_{\min }$ slightly below $0.38 \mathrm{~nm}$, while the gap $d$ in even numbered alkanes is bare $0.125 \mathrm{~nm}$ and in oddnumbered $-0.195 \mathrm{~nm}$, but positronium may still exists there. The geometry of free volume with confinement in one dimension only leads to the results entirely different than for spherical geometry. Reduction of slit thickness $d$ toward zero means that the Ps structure approaches that of a two-dimensional hydrogen-like atom. Such a structure was discussed by many authors, e.g. by Zaslow and Zandler [112]. According to the theory, "flat hydrogen" is four times smaller than three-dimensional one, and its binding energy is four times larger. The interlamellar gap in alkanes is of non-zero size, however in even-numbered ones its thickness is close to two Bohr radii (i.e. less than average distance $3 a_{o}$ of particles forming the Ps atom). The problem is analogous to that of an exciton (electron+hole system) in quantum well in layered solid [113]. In this case the binding energy increases monotonically with decreasing the well thickness, from three-dimensional non-confined structure to two-dimensional one, bound four times stronger, like in the case of hydrogen. At the gap thickness existing in even-numbered alkanes one can expect the binding energy about 1.5 times larger than that of free Ps atom. 


\subsection{Positronium in orientationally disordered crystals}

\subsubsection{Plastic crystals}

Other class of media in which positronium appears at arbitrary temperature are solid plastic crystals. The plastic phase is characterized by an orientational disorder of molecules in all spatial directions (only the translational symmetry of their centers of mass is preserved). The molecules of plastic crystals have a nearly spherical, globular shape. With lowering the temperature such plastic crystals undergo a first order solidsolid phase transition introducing the ordering of orientations (the "rigid phase"). This class of crystals was investigated extensively by Eldrup, Sherwood et al. [72-77] including succinonitrile, camphene, adamantane,

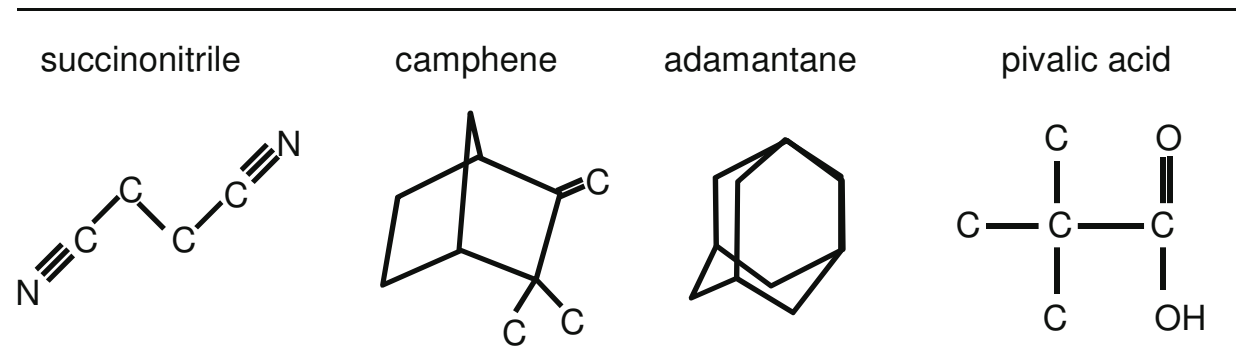

All C-C bonds single (if not drawn otherwise)

$\mathrm{H}$ atoms not indicated

pivalic acid, cyclohexane and cyclooctane. The rigid phase of these crystals shows the existence of one long-lived component only, with $\tau_{3} \approx 1.5 \mathrm{~ns}$, but in the plastic phase with the rise of temperature an additional, longer-living component begins to grow, reaching a saturation intensity close to the melting point. This component was ascribed successfully to o-Ps trapped in vacancies.

In some cases the sum of intensities of two long-lived components was found constant, temperature independent. Thus, the total probability of Ps formation did not change, the rise of temperature enhanced the transitions of o-Ps from the "bulk" states to the vacancies only. Neglecting the possibility of detrapping one receives:

$$
\begin{gathered}
\tau_{3}=1 /\left(\lambda_{b}+K^{\prime}\right) \\
\tau_{4}=1 / \lambda_{V} \\
I_{4}=I \frac{K^{\prime}}{\lambda_{b}-\lambda_{V}+K^{\prime}}
\end{gathered}
$$


where $\lambda_{b}, \lambda_{V}, K, I$ are the decay rate of o-Ps in small defects (dominating in the rigid phase), the o-Ps decay rate in vacancies, the bulk-vacancy transition rate, total intensity of o-Ps, respectively.

Fig.41 shows the fragment of experimental results published by Eldrup et al.[72] for succinonitrile. The solid lines representing the calculations according to Eqs. 43, 45 fit to the experiment very well.

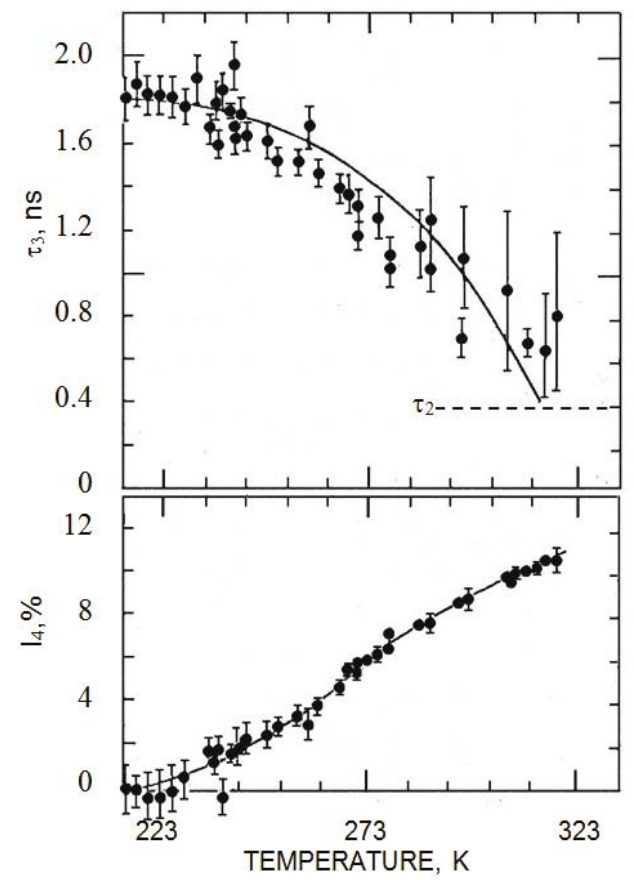

Fig. 41. The ortho-Ps lifetime $\tau_{3}$ and relative intensity $\mathrm{I}_{4}$ in succinonitrile as a function of temperature (from M. Eldrup et al. [72]). The lifetime $\tau_{4}$ was fixed, $\tau_{4}=2.45 \mathrm{~ns}$.

The slope of Arrhenius plot of $\ln K^{\prime}$ vs. $1 / T$ should give the enthalpy of defect formation $H_{v}$. In the case of adamantane and bicyclooctane the enthalpies were found very close to the value of sublimation heat $L_{s}$, as it should be for vacancies; for others they are $(0.5-0.7) L_{\mathrm{s}}$ [114]. As a rule the consistency of $H_{v}$ and $L_{s}$ is observed in the crystals with not too high plasticity.

The data about the volumes of vacancies in plastic crystals known from crystallography, and $\tau_{4}$ lifetimes measured by Eldrup et al. have created the base for the Tao-Eldrup model (exactly for the determination of the $\Delta$ parameter). However, there are some exceptions from the simple relation: void volume - o-Ps lifetime, proposed by this model. To the exceptions belongs methane, which also exists in the plastic

phase in the range from $20.5 \mathrm{~K}$ to the melting point $(91 \mathrm{~K})$. The temperature de-pendence of o-Ps lifetime is similar to that in the crystals listed above, however for the molecular volume of $0.129 \mathrm{~nm}^{3}$ [115] one can expect the o-Ps lifetime in a vacancy to be about $2.4 \mathrm{~ns}$, while such lifetime is observed already in the brittle phase and grows to $2.9 \mathrm{~ns}$ in the plastic one near the melting point [116]. It can be the result of very soft structure of that solid. The studies by Brillouin scattering [117] indicate that the $\mathrm{CH}_{4}$ molecules in the plastic phase of methane rotate almost freely, the properties of this phase are liquid-like, thus, methane can be 
the case analogous to solid helium: Ps zero-point motion of positronium forces an additional free volume. This effect will be described in detail in the Section 7.

\subsubsection{Rotator phase}

In the alkanes with odd-numbered carbon chains $9 \leq \mathrm{n} \leq 39$ and even-numbered ones in the range $22 \leq \mathrm{n} \leq 40$ a "rotator" phase exists in the temperature interval from 1 to $12 \mathrm{~K}$ below the melting point. In the rotator phase the translational symmetry of the long molecular axes in the crystal is preserved, yet the molecules can rotate round these axes (orientational disorder limited, in comparison to plastic crystals). Due to mechanical properties of phases the low-temperature one is called "brittle" or "rigid", the rotator phase is "waxy". In the rotator phase the o-Ps lifetime is evidently longer than that in the rigid one (Fig. 42) due to the presence of nonplanar conformers.

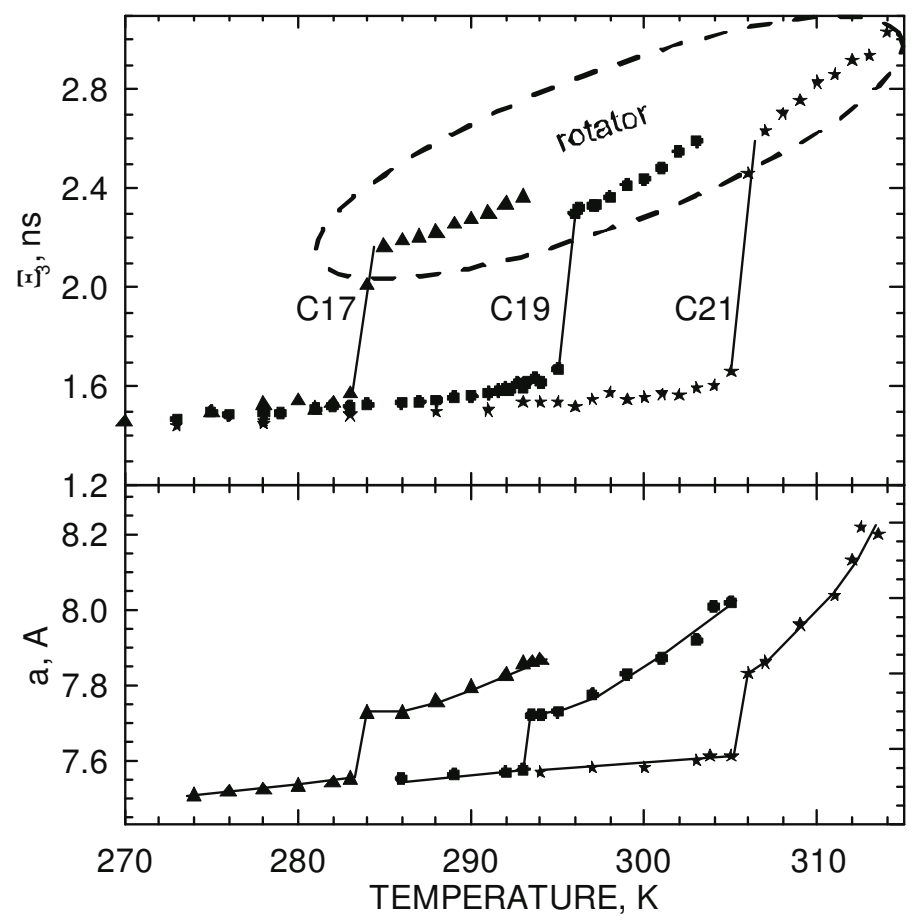

Fig. 42. Top - temperature dependence of the o-Ps lifetime in the range of rotator phase for several odd-numbered n-alkanes (C17, C19, C21), bottom - temperature dependence of the crystalline axis $a$ for the n-alkanes shown above. Crystallographic data from [118]. 
They are mainly of end-gauche and kink type, their concentration rises with the length of chain (and temperature), e.g. for $\mathrm{C} 17$ the concentration of kinked molecules is about 8\%, while for C29 it approaches $70 \%$ [118]. Mismatching the kink conformers and all-trans ones leads to the creation of free volumes inside the lamella. The nonplanar character of kink conformers forces an increased spacing of neighbouring molecules, which is seen in crystallographic data - increase of the crystal cell in the direction of the $a$ axis, perpendicular to the molecular chain plane. In Fig. 42 the correlation of $\tau_{3}$ lifetime and length of the $a$ axis is clearly visible. The shape of free volume near the kinked molecule is expected to be elongated, approximately half of the molecule length (the "kink" can move along the molecule, but its average position is in its centre). For a given alkane both parameters, $\tau_{3}$ and $a$, rise with temperature. At a fixed average length of the void, an increase of its volume can appear owing to the increase ofits cross section only. It is possible at a high concentration of kink conformers, when one can find two such molecules side-by-side. This gives an enlarged free volume (Fig.43); the number of such pairs rises with temperature.

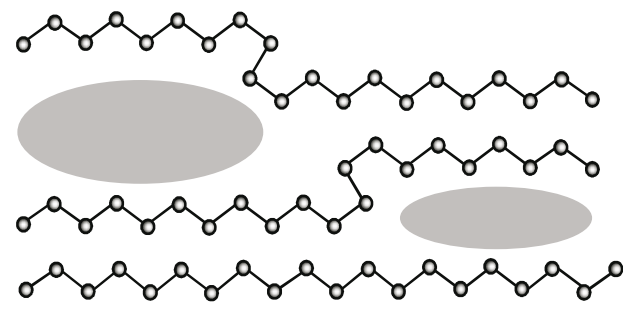

With increasing length of the carbon chain the o-Ps lifetime in the rotator phase rises, but only up to certain maximal value, identical with the lifetime in the liquid phase and independent on the chain length. An example is shown in Fig. 44. The lifetime $\tau_{3}$ in C30 does not change at melting, while the phase transition rotator - liquid is well marked as the skip of intensity $I_{3}[119]$.

In fact, there are five versions of the rotator phase in alkanes. In some of them, depending on temperature, one can observe different rotator phases, e.g. phases RI or RII for $22 \leq \mathrm{n} \leq 26$, and phases RIII, RIV for $27 \leq \mathrm{n} \leq 30$. However, the PALS method is not sensitive enough to detect these differences. Lifetime $\tau_{3}$ and intensity $I_{3}$ change with the structure modification; a more radical rebuilding of the structure should result in more distinct changes of these parameters. The enthalpy of transition can be a certain measure of structural modification. The 
enthalpy of transition rotator - rigid crystal is about $(30 \div 50) \mathrm{kJ} / \mathrm{mol}$ depending on chain length, while e.g. of the transition RI - RII in C25 it is $0.2 \mathrm{~kJ} / \mathrm{mol}$ only [120].

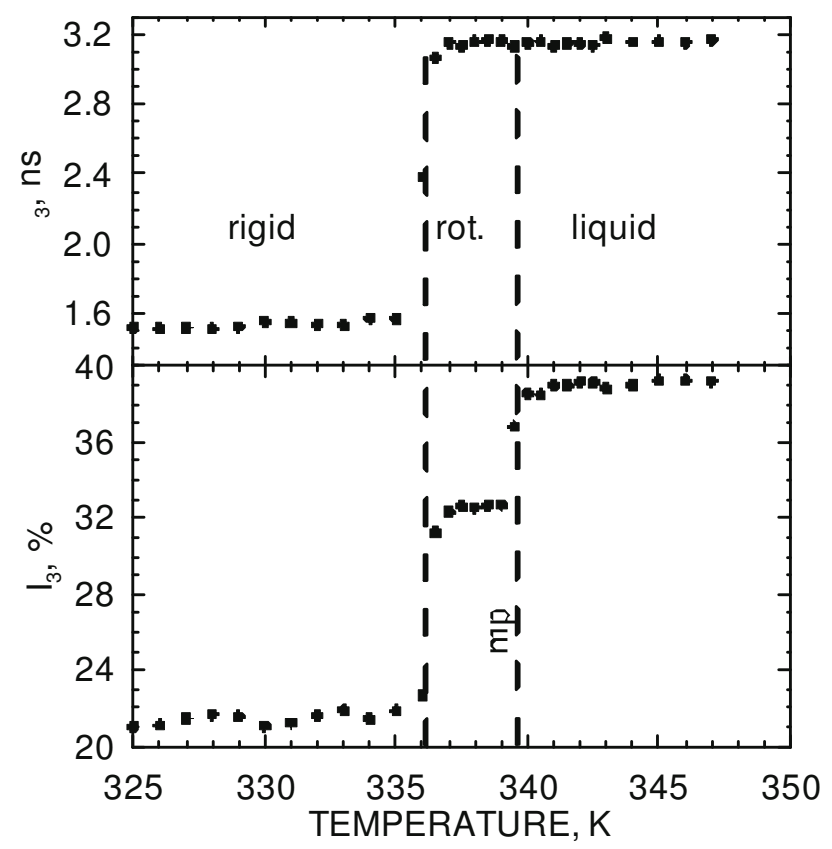

Fig. 44. Temperature dependence of o-Ps lifetime in n-triacontane C30. From Ref. 119.

The phase transitions can be induced by pressure too. Application of pressure to the sample in rotator phase leads to the same effect as lowering the temperature. In alkanes increasing the pressure by $1 \mathrm{MPa}$ is equivalent to decreasing the temperature by $0.25 \mathrm{~K}$. The lifetime and intensity obtained for different values of temperature and pressure for $\mathrm{n}$ nonadecane are compared in Fig. 45.

The rotator phase is observed also in even numbered long-chain alcohols with $n \geq 12$, while in alkanes with $n \geq 22$. In alcohols it appears only at cooling the sample from the melt; in heating runs this phase is not present. Like in alkanes, the rotator phase contains a large concentration of nonplanar conformers (end-gauche and kink ones). It results in an increased o-Ps lifetime compared to the low-temperature rigid phase, but the intensity of o-Ps is smaller than in both neighbouring phases. This can be explained by an increased dielectric permittivity $\varepsilon$ of the rotator phase [121]. 


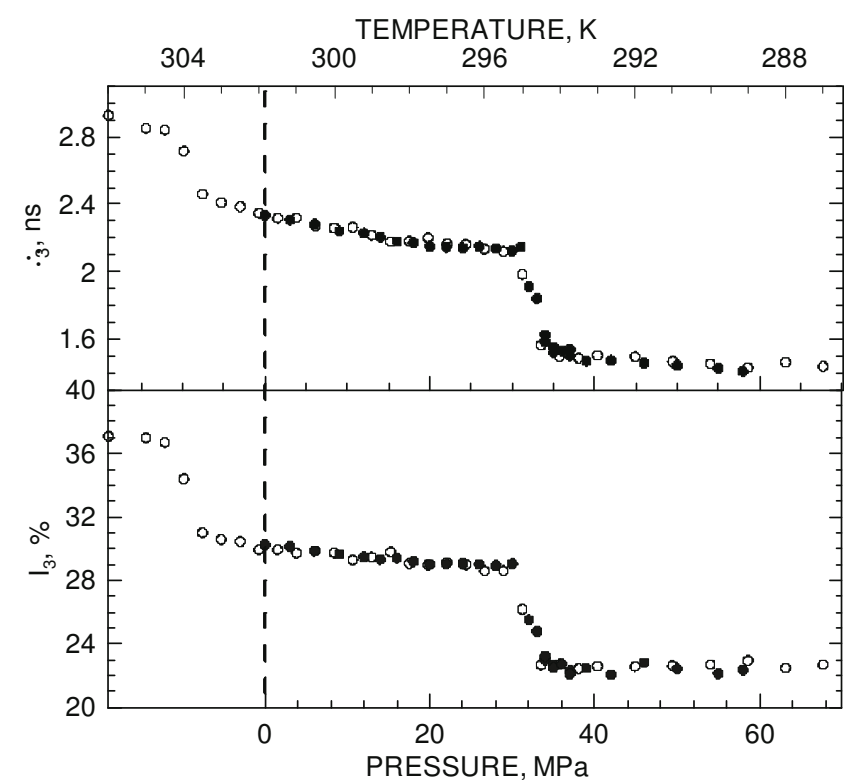

Fig. 45. A comparison of pressure (at $302 \mathrm{~K}$, dots) and temperature (at $<<0.1 \mathrm{MPa}$, open circles) dependences of o-Ps lifetime and intensity in n-nonadecane $\mathrm{C} 19$. The temperature abscissa is inverted, scaled $1 \mathrm{~K}=4 \mathrm{MPa}$. Melting point of $\mathrm{C} 19$ is $304 \mathrm{~K}$.

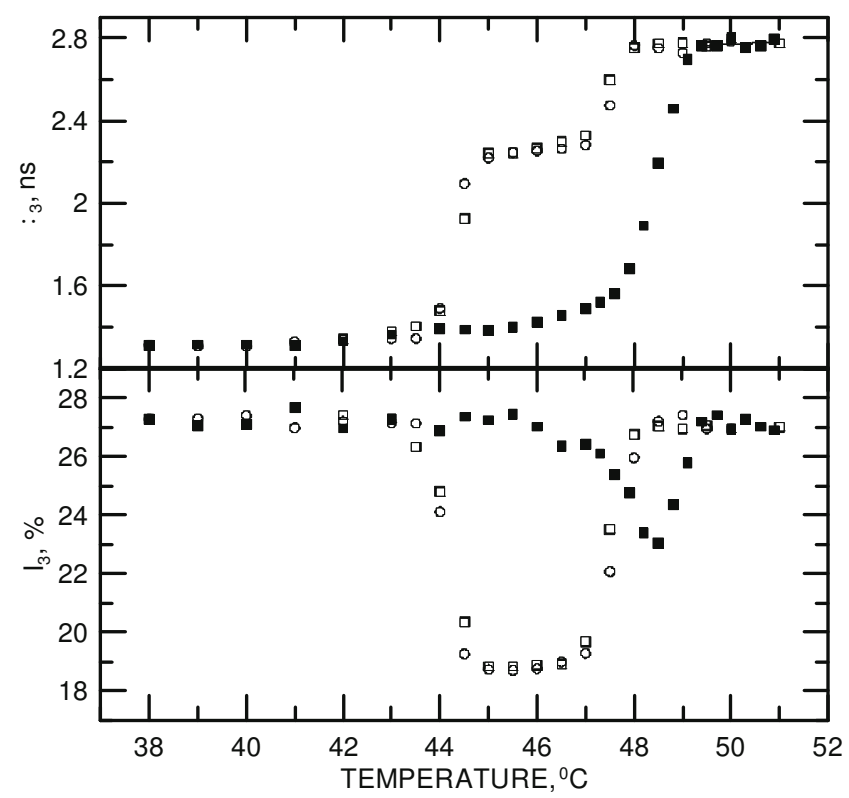

Fig. 46. Temperature dependence of the o-Ps lifetime and intensity in n-hexadecanol-1. Full symbols - temperature up, open symbols - temperature down. The hysteresis of about $1.5 \mathrm{~K}$ is seen. 
In the simple version of the spur model a larger $\varepsilon$ means a reduction of the Onsager sphere radius (Eq.15) and diminishing of the Ps formation probability. A typical dependence of the o-Ps lifetime and intensity on rising and decreasing temperature is shown for the case of n-hexadecanol-1 [122] in Fig.46.

\subsection{Positronium in solid phases of long chain alkanes}

When an odd numbered chain contains over 25 carbon atoms, new solid phases, preceding the rotator phase, appear. In the alkanes with $\mathrm{n} \geq 25$ there is a phase denoted as IV, which is visible in PALS by an increase of $\tau_{3}$ lifetime by about $0.2 \mathrm{~ns}$ (Fig. 47), this increase is due to a large concentration of end-gauche conformers; shortened molecules increase the average width of interlamellar gap.

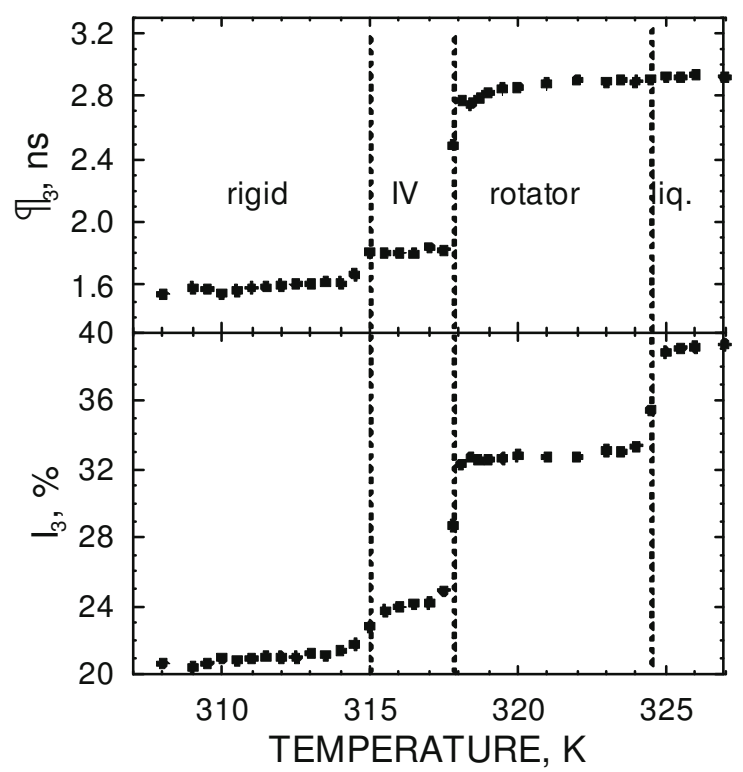

Fig. 47. The o-Ps lifetime and intensity in $\mathrm{C} 25$ as a function of temperature. (B. Zgardzińska, Ph.D. Thesis UMCS Lublin, 2008)

In even numbered alkanes next phases appear from $n=36$ upwards. The crystalline structure of these high-n alkanes can depend on the way of sample preparation: crystallization from the solution, solidification of the melt etc. At heating the samples obtained by crystallization one observes the transition from the low-temperature monoclinic phase to the phase $\mathrm{C}$, also monoclinic but with much larger 
tilt angle. For the chain length of $n$ ranging from 38 to 44 the transition is well visible, although the step of $I_{3}$ at the transition point decreases with increasing $n$ and above $n=46$ disappears; $I_{3}$ changes smoothly with temperature. Phase $\mathrm{C}$ is characterized by the Ps intensity much lower than in the rotator phase (which appears up to $\mathrm{C} 38$, but not for longer chains), and also by shorter o-Ps lifetime (Fig. 48).

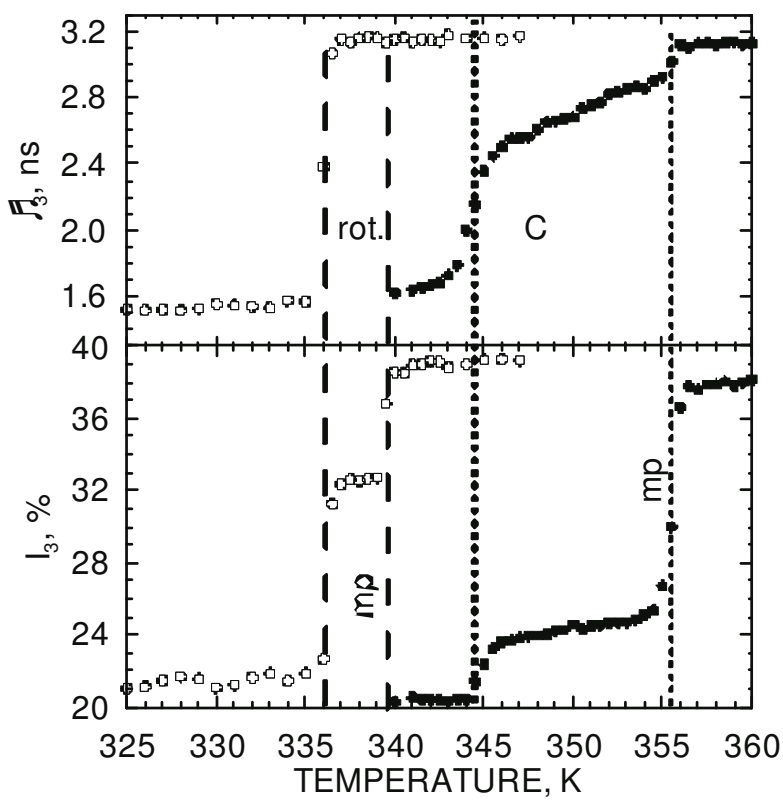

Fig. 48. Temperature depen-dence of o-Ps lifetime and intensity in n-tetracontane $\mathrm{C} 40$ (dots; melting preceded by the $\mathrm{C}$ phase). For comparison similar dependence for $\mathrm{C} 30$ is shown (open symbols, melting preceded by rotator phase). From Ref. 111 and 119.

In PALS measurements, an increase in the o-Ps lifetime is usually a signal of growing disorder. For example, in alkanes the number of intramolecular defects (gauche bonds introducing nonplanar form of molecules) rises with the chain length. A relatively shorter lifetime in phase $\mathrm{C}$ indicates a reverse effect. Contrary to the rotator phase, in which for long chains the o-Ps lifetime is practically independent on temperature and equal to that in the liquid, in phase $\mathrm{C}$ that lifetime rises continuously from about $2.4 \mathrm{~ns}$ to $2.8 \mathrm{~ns}$ near the melting point. The step of $\tau_{3}$ at melting, invisible when the preceding phase is the rotator one, for the transition $\mathrm{C} \rightarrow$ liquid is well marked [111]. Better ordering is confirmed by Kim et al. [123] who have investigated the gauche bond density $m_{g}$ (the number of such bonds per one molecule) by IR spectroscopy. In the 
rotator phase of C36 $m_{g}=0.7$, whereas for C phase in C40 it surprisingly goes down to about 0.4. The $\mathrm{C}$ phase is thus better ordered than the rotator one. In C38 and next even numbered alkanes the solid-solid transition becomes diffused, the transition region covers several $\mathrm{K}$, and finally for $n \geq 50$ it is seen as a change of the $d \tau_{3} / d T$ only; an analogous smooth dependence on temperature is seen in $m_{g}$ [123].

\section{POSITRONIUM IN LIQUIDS}

\subsection{The bubble model}

Positronium in condensed media needs a sufficiently large free volume to be localized there. In liquids the natural intermolecular empty volumes can be estimated e.g. from the sound velocity [124]; their sizes calculated by Ujihira et al. [125] are found below $0.1 \mathrm{~nm}$. Even if one accounts the stochastic fluctuations of molecular positions, the probability of the appearance of voids exceeding $0.2 \mathrm{~nm}$ is very low, i.e. there are no free volumes which can accommodate Ps atom (see Eq.18). On the other hand, already the early experiments with liquid helium [126, 127] have shown that in this liquid positronium exists and the o-Ps lifetime is unexpectedly long, $\tau_{3}=91$ ns (large values of this lifetime were seen also in other cryogenic liquids). To explain such long lifetimes, Ferrell [128] has proposed the bubble model. The pressure exerted by zero-point motion of Ps creates a spherical cavity (bubble) around it, the equilibrium radius $R$ is determined by the minimum of energy:

$$
\frac{d}{d R}\left[E_{P_{s}}(R)+4 \pi R^{2} \sigma+4 \pi R^{3} p / 3\right]=0
$$

where $E_{P_{s}}$ is the Ps energy in the bubble, $\sigma$ - the surface tension, $p$ - the external pressure.

The bubble model was further developed by Roelig [129] and Buchikhin et al.[130]. The trapping of Ps in voids looks differently in solids and liquids. In solids Ps needs preexisting regions of low (zero) electron density to appear as a stable structure, while in liquids it produces its own cavity - the bubble. Thus, positronium in liquids is not strictly a probe; annihilation experiments bring the information about the extent of perturbation of the medium in the vicinity of the Ps atom, not about the unperturbed structure.

There is a rich set of experimental data about the o-Ps lifetime in organic liquids, measured by Mogensen and Jacobsen [131], however, at 
the room temperature only; the data about temperature dependences are much more limited. Having the bubble radius estimated from the TaoEldrup or similar model one can try to find the surface tension at the curved bubble surface. The ratio of surface tension of droplet $\sigma$ to that at flat surface of liquid $\sigma_{\infty}$ is given by Tolman [132]:

$$
\frac{\sigma}{\sigma_{\infty}}=\frac{1}{1+2 \delta / R}
$$

where $\delta$, called Tolman's length, is the difference of radius determined by vacuum-bulk boundary and the radius at which acts the surface tension. The value of $\delta$ is positive, the surface tension acts somehow slightly "behind" the surface. If so, the surface tension of a droplet is smaller than for flat surface, while of the bubble - larger [133,134]. The Eq. 47 can be written as:

$$
\frac{\sigma}{\sigma_{\infty}}=\frac{1}{1+2 \delta \kappa}
$$

where $\kappa$ means curvature (positive for droplets, negative for bubbles).

The Eq. 46 contains the term $E_{P S}(R)$ which can be calculated by standard quantum mechanical methods [135], however, this way we have no analytical formula which could be inserted into Eq.4 6 and differentiated. Certain approximations are necessary.

One of possibilities is to apply the same mathematical trick as in the Tao model [71]. i.e. to substitute the well of depth $U$ by an infinite one but with the radius broadened by $\eta$ chosen to give the same value of the Ps energy [136]:

$$
E_{P s}(R, U)=E_{P s}(R+\eta, \infty)
$$

In this approximation

$$
E_{P s}(R+\eta, \infty)=\frac{\hbar^{2} \pi^{2}}{4 m_{e}(R+\eta)^{2}}
$$

Fig. 49 shows the comparison of $E_{P S}$ calculated exactly for three different rectangular potentials $U=1.0 ; 1.5 ; 2.0 \mathrm{eV}$ with these values for infinite depth and $\eta$ fitted to get identical values as for a finite depth version at $R=0.4 \mathrm{~nm}$ (i.e. in the middle of a radius range appearing in the case of Ps in liquids). The deviation of $E_{P S}$ calculated from Eq. 50 from the value expected for a realistic well depth in the $R$ range $(0.35-0.46)$ $\mathrm{nm}$, i.e. the lifetimes (2-6) ns, does not exceed $2 \%$. 


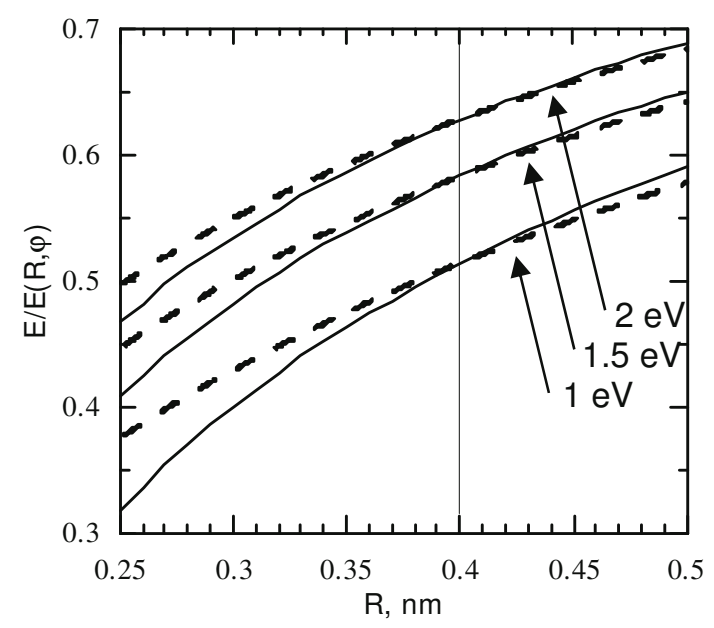

Fig. 49. Ps energy in a rectangular potential well [136]. Continuous lines are calculated for well depth $U=1 ; 1.5$ and $2 \mathrm{eV}$. The dashed lines for an infinite depth and the well broadened by $\eta$ to match the exact calculation at $R=0.4$ $\mathrm{nm}$. From top to bottom the $\eta$ values are $0.105 ; 0.124$ and $0.158 \mathrm{~nm}$. The ordinate is in units of Ps energy in an infinite, not broadened well $\mathrm{E}(\mathrm{R}, \infty)$.

Eq. 46, neglecting the term related to pressure (smaller by two orders of magnitude at normal pressure), is now:

$$
\frac{d}{d R}\left[\frac{\hbar^{2} \pi^{2}}{4 m_{e}(R+\eta)^{2}}+4 \pi R^{2} \sigma\right]=0
$$

After differentiation we get the fourth degree algebraic equation. If we assume, following the existing literature data, $U=1.0 \mathrm{eV}$ and the radii according to the Tao-Eldrup model, then for alkanes near the melting point we obtain the Tolman's length 0.05 to $0.06 \mathrm{~nm}$, i.e. of the order of atomic radius [137]. The surface tension of the Ps bubbles is increased 1.4 to 1.5 times compared to a flat surface.

The minimum of energy in Eq. 51 is very flat and shallow with respect to the thermal energy $k T$, thus one should not expect a precisely fixed radius but a fluctuating one; such fluctuations can be responsible for the effects observed by Gregory and Chai in hexane [138].

\subsection{Molten alkanes and alcohols}

An interesting property of liquid alkanes is an identical o-Ps lifetime near the melting point independently on the length of the carbon chain. With the rise of temperature that lifetime rises too, the dependence of $\tau_{3}$ on the temperature above the melting point is very similar for all alkanes under study, from heptane C7 to hexatriacontane C36 (Fig.50), with a slight tendency to decrease the slope $\mathrm{d} \tau_{3} / \mathrm{dT}$ with increasing molecular length [137]. 


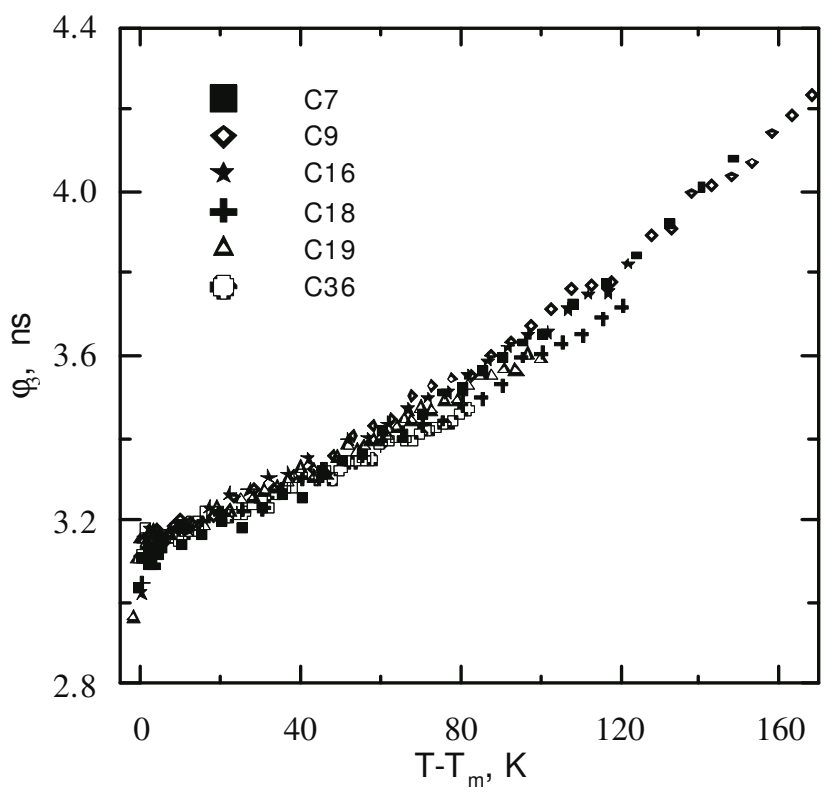

Fig. 50. The o-Ps lifetime in n-alkanes as a function of temperature above their melting points. Symbols explained in the figure [137].

The rise of $\tau_{3}$ with temperature reflects the common property of liquids - decrease of surface tension. An exception is water: in spite of decreasing surface tension the o-Ps lifetime also decreases [139, 140]. Stepanov et al. [141] propose to explain this behaviour as the effect of exponential increase of the rate constant of the Ps oxidation reaction by intratrack $\mathrm{OH}$ radicals and $\mathrm{H}_{3} \mathrm{O}^{+}$ions.

In the cases described above, the pressure over the liquid sample was produced by own saturated vapour, always well below the atmospheric one. An exception is the case published by Jacobsen et al.[142], where the sample in a closed vessel was heated up to the critical point. In neopentane (2,2-dimethyl propane) the temperature and pressure at critical point are $433.4 \mathrm{~K}$ and $3.13 \mathrm{MPa}$, respectively. The o-Ps lifetime in the sample near the critical point reached $20 \mathrm{~ns}$ and at further heating was changing very little (Fig. 51). Above the critical point there are no "bubbles", the o-Ps lifetime depends on the number of collisions per time unit. The dependence of decay constant on temperature below $413 \mathrm{~K}$ is well described by the function $\lambda_{3}=\operatorname{Aexp}(-b R)$, where $R$ is the bubble radius determined by Eq. 46. Analogous measurements were performed by Jacobsen et al. [143] for sulphur hexafluoride. 


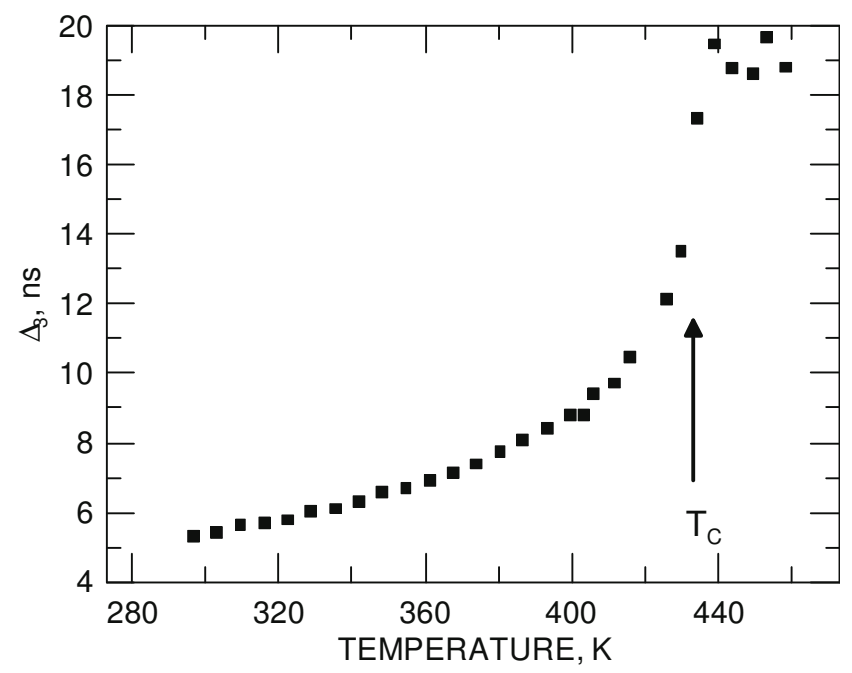

Fig. 51. The o-Ps lifetime in neopentane as a function of temperature . The arrow indicates the critical temperature. From: F.M. Jacobsen, Ris $\emptyset$ Report 433.

When over the sample an inert gas is present, it dissolves in liquid. The solubility is proportional to the gas pressure (Henry's law; valid at molar concentrations of solute not exceeding $\sim 3 \%$ ). Dissolved gas changes the surface tension of liquid, that reflects in observed o-Ps lifetime. Having the bubble radius calculated from Tao-Eldrup (or other) model one can determine the surface tension as a function of pressure. As an example the system alkane (nonadecane) + argon is shown in Fig. 52. The pressure dependence of $\sigma$ can be described by the function:

$$
\sigma=A+B \exp (-C p)
$$

Particular case of oxygen atmosphere will be described in Sec.9.

In liquid primary alcohols (1-alkanols) the o-Ps lifetime dependence on temperature above the melting point is similar to that in alkanes: monotonous $I_{3}$ rise, weakly dependent on the length $n$ of carbon chain. Along the homologous series the change of $I_{3}$ intensity with temperature shows some peculiarities: in the shortest chain alcohols $I_{3}$ is almost independent on temperature, but then $d I_{3} / d T$ increases systematically with rising chain length $n$. Usually the o-Ps intensity in solid phase is larger than in liquid one, but that step diminishes with $n$ up to the change of its sign at $n=16$ [137]. 


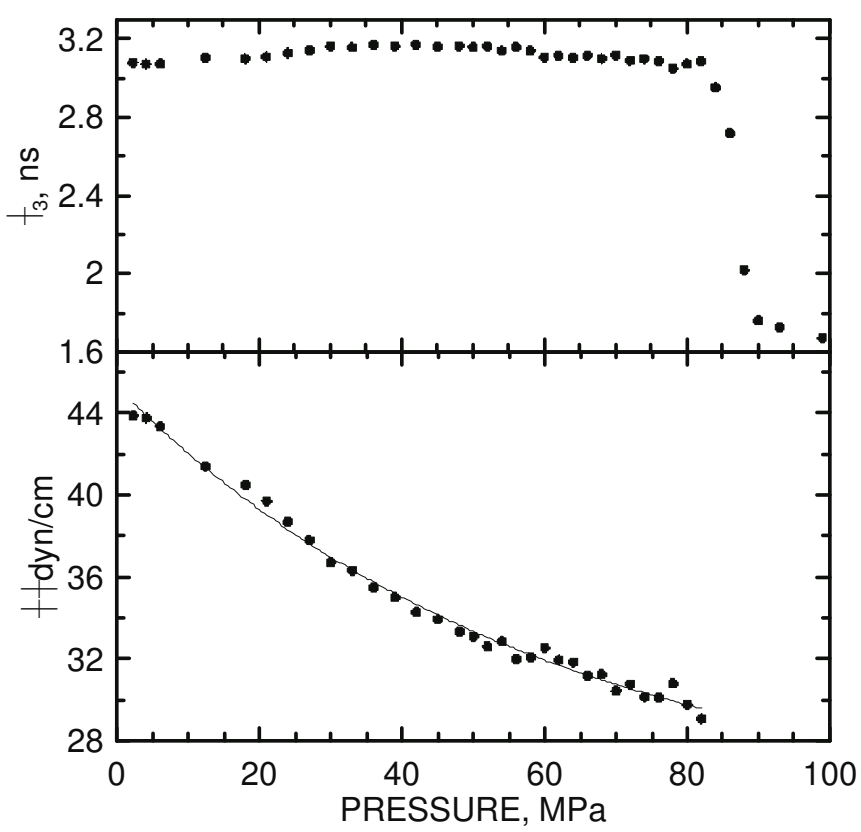

Fig. 52. Pressure dependence of the o-Ps lifetime (top) and surface tension (bottom) in nona-decane + argon system

\subsection{Supercooling}

In the case of the supercooled liquids the PALS spectroscopy allows to observe the rate of transition liquid-solid. For example, in butanol [144] directly after rapid cooling from $188 \mathrm{~K}$ (5 K above the melting point) to distinctly lower temperatures, the o-Ps lifetime in supercooled liquid is shortened due to increased surface tension (Fig. 53). The liquid is not stable and (at fixed temperature) solidifies, the o-Ps lifetime and intensity decrease to the values observed in the solid phase.

The lower the temperature, the faster is the transition, however, in the case of butanol the half-time of transition reaches a minimum $(0.1 \mathrm{~h})$ at about $146 \mathrm{~K}$. At supercooling to still lower temperatures the transition time rises again, at $120 \mathrm{~K}$ it exceeds $10 \mathrm{~h}$. This can be due to the large viscosity of butanol being still liquid (several scores of $\mathrm{K}$ below the melting point) and thus slowing down the rearrangement of molecules. 


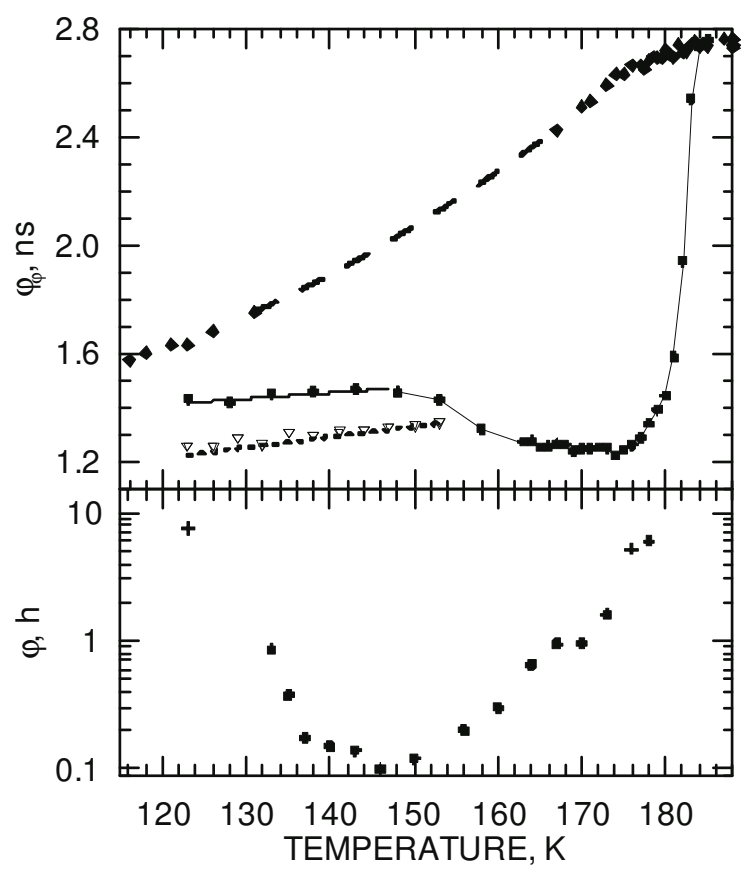

Fig. 53. Top: temperature dependence of the o-Ps lifetime in supercooled butanol. Diam-onds are for the liquid directly after cooling; the lower solid curve is the lifetime after termination of transition. Triangles and dotted line denote the lifetimes for the sample solidified at $173 \mathrm{~K}$ and then cooled down. Bottom: half-time of the transition. (based on [144]).

Development of solidification after rapid cooling from $188 \mathrm{~K}$ to $173 \mathrm{~K}$ is shown in Fig. 54. Due to high rate of transition it was necessary to reduce the time of data collection to $12 \mathrm{~min}$ per point (at still lower temperatures the time of data collection was reduced further). Resulting low statistics forced to quit the spectrum decomposition and assume as a measure of the o-Ps intensity the total count number $Q$ above 2 ns. The dependence $Q$ vs. time in Fig. 54 can be described by the function:

$$
Q=A \exp (-B t)^{n}+C
$$

where $C$ is the final count number in the solidified sample; the exponent is found equal $n=(4.2 \pm 0.2)$. Eq. 52 has the form of Avrami function [145]. Exponent $n=4$ in Avrami function is the maximal one and means that there are no limitations of solidification in all three dimensions and the preexistence of nucleation sites is not needed. 


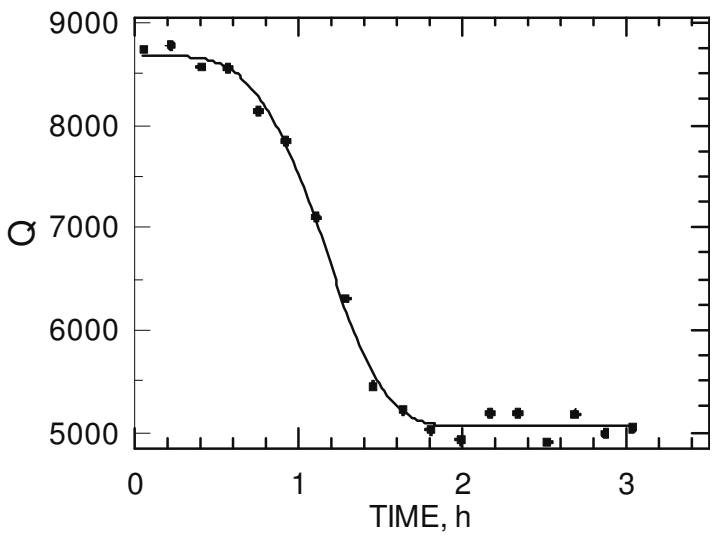

Fig. 54. Area $Q$ of the tail of lifetime spectrum $(t>2 \mathrm{~ns})$ in butanol as a function of time after lowering the temperature to $173 \mathrm{~K}$.

Observation of the phase transition at $123 \mathrm{~K}$ shows that the decrease of lifetime stops at $1.42 \mathrm{~ns}$, while the lifetime in the solid, as measured with the sample solidified at $173 \mathrm{~K}$ and cooled to $123 \mathrm{~K}$ (triangles and dashed line in Fig. 53), should be $\approx 1.25$ ns. In the range from $120 \mathrm{~K}$ to $135 \mathrm{~K}$ the transformation process stops before full solidification. This effect is known as "frustration". The sample consists of solid domains and liquid regions. Intermolecular interaction by hydrogen bonding of the butanol molecules can, at so low temperatures, suppress the rearrangement into regular crystal [146]. In the measurements analogous to that shown in Fig. 54, but performed at $123 \mathrm{~K}$, the exponent in Avrami function dropped to $n=(2.5 \pm 0.3)$, indicating limitations in the transformation process. This value of $n$ is close to that found in Ref. 146 by Raman spectroscopy: $n=2.7$. Both measurements, PALS and Raman spectroscopy estimate that at $123 \mathrm{~K}$ the solid fraction is over $70 \%$.

\subsection{Definition of void radius}

The Tao-Eldrup equation gives the relation between void radius and o-Ps lifetime. The situation looks simple in the case of liquids, where the spherical shape is a natural one. However, the radius is rather poorly defined, in the majority of cases it is simply an equivalent radius only. Even in spherical voids, precising the void definition is important when the transition region from large to negligible electron density is comparable to $\Delta$ parameter. In the papers by Eldrup et al. [72-77] on plastic crystals the Wigner-Seitz molecular radius was used, i.e. the radius of a sphere, which volume is equal to the volume of elementary crystal cell divided by the number of molecules per cell (not the radius of roughly globular molecule alone). Another approach is proposed by Zhao 
and Ujihira [147] for liquid crystals and Jasińska et al. [148] for crystalline solids. The well radius is assumed by them as the radius of maximal sphere tangent to surrounding atoms. This in turn needs to define the atomic radii. In Refs.147,148 the atomic radius was assumed as a distance from the centre to the maximum of electron density at the outermost orbital of free atom. For anthracene, azulene, acenaphthylene, the radii of inscribed spheres were found below $0.12 \mathrm{~nm}$ [148], thus lie well below $R_{\min }$ (Eq. 18) for potential depth $U<3 \mathrm{eV}$. Even if the atomic radii are not chosen properly the result helps to explain why in these solids positronium is not observed (until melting).

There is to stress, that in the discussion about positronium in liquid the term "radius" is used in several different meanings: vacuum-bulk dividing radius, surface tension radius (difference of these two radii is just the Tolman length), Ps potential well radius (following from the approximation of real potential shape by a step-wise one). The values of radii are very similar, but not identical, and in the scale of a fraction of nanometer the use of one "universal" radius can lead to inaccuracies.

\section{POSITRONIUM IN POLYMERS}

\subsection{Size and number of free volumes}

The disordered structure of polymers creates a variety of free volumes. Fig.55 shows the comparison of the PAL spectrum in an acenaphthylene monomer and in the same medium after beginning of polymerization. An intense long-lived component appears already at a mean molecular mass 352 , i.e. about twice of that for a monomer, when dominant molecules are dimers, while in monomer there is no posi-

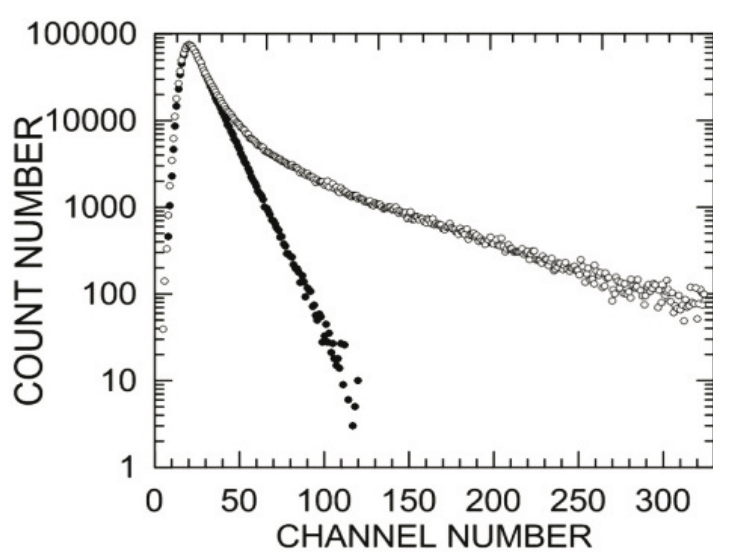
tronium at all (further polymerization does not influence substantially the intensity of that component).

Fig. 55. Lifetime spectrum of acenaphthylene monomer, molecular mass $\mathrm{M}=152$ (dots) and of the same sample after beginning of polymerization, $\mathrm{M}=352$ (open symbols). 
The structure of empty volumes in polymers is more complex than in crystalline solids. One has to account for the fact that two types of these volumes exist: pre-existing static holes and dynamic ones caused by vibrations, rotations of chain elements, molecular relaxation etc. According to the terminology used in polymer science, the latter ones only are called "free volume". It means that a certain amount of the volume characterized by negligible electronic density is not accounted for as free volume. Positron and positronium lifetimes are of the order of $10^{-10}-10^{-9} \mathrm{~s}$, thus static holes and dynamic ones existing the time longer or comparable to the lifetime of positron probe can become trapping sites for positronium.

The concept of free volume, which appeared in theories as early as 1930s [149] and was developed further in '50s, plays an important role in the description of many properties of polymers, like viscosity, elasticity, glass transition, mechanical properties, gas permeability etc. The classic polymer studies deal with mainly macroscopic, bulk properties, while positron lifetime spectroscopy is a unique technique allowing to determine the individual free volume hole sizes and concentrations.

It is important to know not only the average size of free volume holes, but also their distribution. In the amorphous structure of a polymer the lifetime spectrum becomes complex, a continuous distribution of void sizes is expected and thus also a continuous distribution of annihilation rates $s(\lambda)$. The PALS spectrum has now the form:

$$
C(t)=\int_{\lambda_{\text {th }}}^{\lambda \max } s(\lambda) \lambda e^{-\lambda t} d \lambda+\sum_{i=1}^{2} I_{i} \lambda_{i} e^{-\lambda_{i} t}+B
$$

(the convolution of the spectrum with the resolution function $\mathrm{P}(\mathrm{t})$ is omitted for simplicity), $\lambda_{t h}$ is the decay constant for smallest free volume detectable by positron method. The sum in the second term describes the annihilation of p-Ps and of free positrons. The use of a discrete $\lambda_{1}$ in the case of p-Ps is a good approximation, while a discrete value of $\lambda_{2}$ for free positrons in organic media is still disputable (if they are really free, not trapped or bound to molecules, only one discrete value of $\lambda_{2}$ should appear).

In the most simplified version of spectrum processing the continuous $s(\lambda)$ is substituted by two (or even one) discrete components and analysed in classic way using e.g. the POSITRONFIT program [35]. More realistically the shape of $s(\lambda)$ can be reconstructed using the MELT program [37] described in Sec. 3.1. In this case all components, including p-Ps and free positron annihilation are in the form of quasi-continuous 
distributions (the procedure cannot fit discrete $\lambda \mathrm{s}$ ). The MELT calculations need very high statistics and $10^{7}$ coincidences or more have to be collected in the spectrum. One has to remember that in the programs like MELT the regularizer value, i.e. entropy limit is chosen by the person processing the spectrum, thus the final result can slightly depend on his will.

Usually the $s(\lambda)$ function has the form of bell shaped peaks (see Fig. 40 [111]), thus Kansy [36] came up with an idea to assume a priori that the $\lambda$ spectrum consists of some log-Gaussian peaks (lifetime $\tau_{p}$ at maximum, $\sigma$ the width of Gaussian) plus some discrete components. This way one can obtain a quite good spectrum description at even moderate statistics of measurement.

Standard polymer investigations by positron methods consist in observation how the polymer structure changes with temperature, pressure or interaction with gases. The sensitivity of Ps spectra in polymers to temperature and pressure was noticed in the earliest papers on positron annihilation. Bell and Graham [4] have observed the decrease of o-Ps yield and shortening of o-Ps lifetime in Teflon with lowering temperature, while De Zafra and Joyner [103] demonstrated the broadening of narrow component in ACAR under high pressure.

Lengthening of the lifetime with temperature is related to the expansion of a polymer. The dimensions of voids rise much faster than it would follow from the thermal expansion coefficient. The latter characterizes the sample as a whole, in which the free spaces occupy a small fraction of the total volume. At various polymer treatments the majority of volume change occurs just in "free spaces". At gas swelling of the polycarbonate (see below) the total sample volume increases by $0.95 \%$, while the free volume - by $14.3 \%$ [150]. The "hole fraction" $h=V_{f} / V\left(V_{f}-\right.$ the total free volume, $V$ - the sample volume) lies in the range from 0.06 to 0.28 . Particularly large free volumes appear in polymers whose chains contain bulky elements such as cyclic structures or side branches, preventing close approach of chains.

Application of high pressure reduces the free volumes and increases the density of occupied one too (keeping in mind that "occupied" volume contains also some empty regions), thus both lifetimes, of o-Ps and of free positrons, diminish with pressure, as can be seen in Fig.56 presenting the data by Dlubek et al. [151] for PTE fluoroelastomer. 


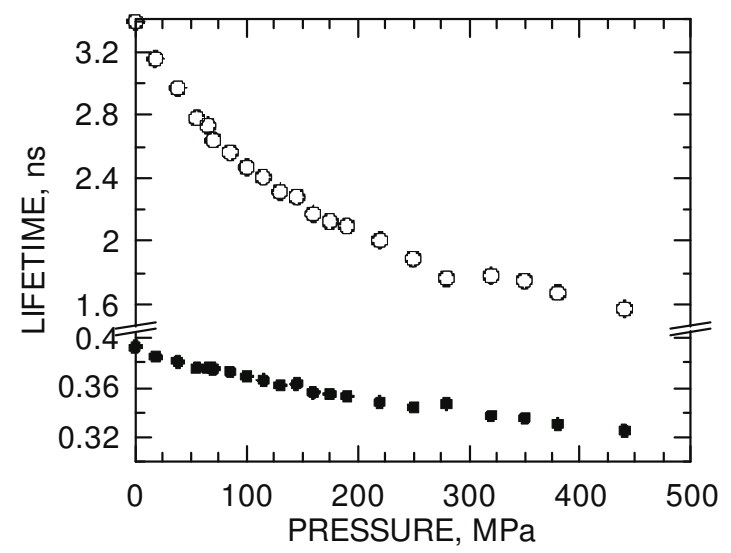

Fig. 56. Pressure dependence of $\mathrm{o}-\mathrm{Ps}$ (circles) and free positron (dots) lifetimes for PTE fluoroelastomer (compiled from [151]).

A characteristic feature of polymers is the phase transition from glassy (brittle) state to soft (rubbery) one. At the transition point $T_{g}$ the expansion coefficient changes by a factor of 5 . The PALS measurements as a function of temperature show the distinct change in the slope of the o-Ps lifetime or of the S parameter at $T_{g}$ (Fig.57).
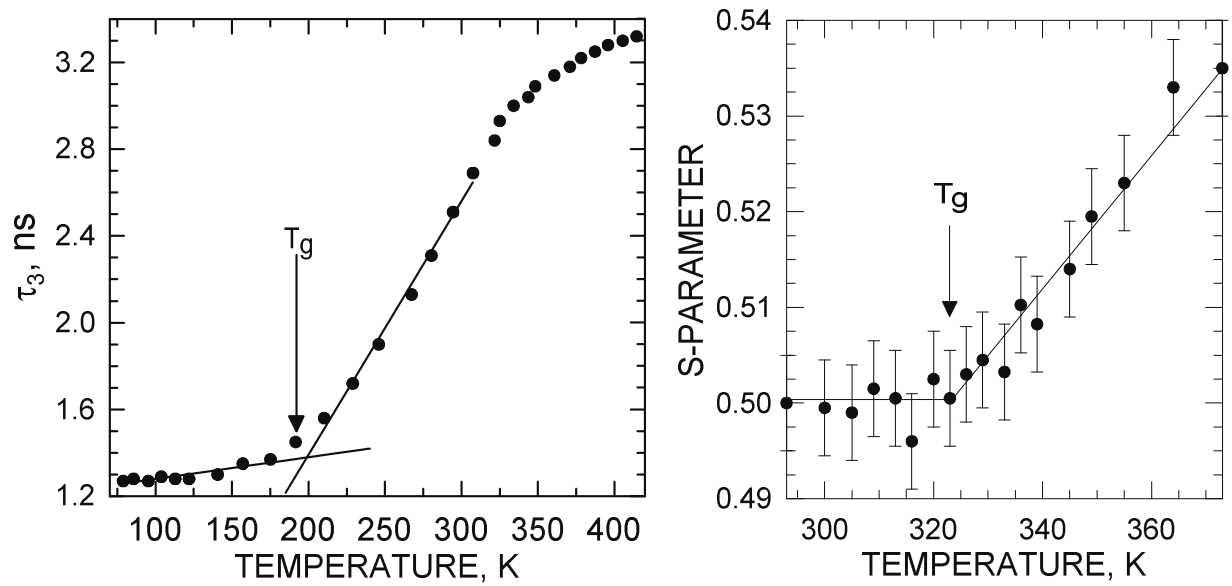

Fig. 57. Left: temperature de-pendence of the o-Ps lifetime in polyisobutylene polymer. Based on the data from [152]. Courtesy of Frans H.J. Maurer. Right: temperature dependence of S parameter in Epidian $5+$ terphene-maleic anhydride.

With the increase of temperature the o-Ps lifetime in the rubbery phase rises initially fast, but then above $(1.2 \div 1.5) T_{\mathrm{g}}$ the slope of temperature dependence is again strongly reduced, although in the macro scale no reduction of thermal expansion is observed. Winberg at al.[152] have found that at this temperature range the polymer behaves like a liquid, the o-Ps lifetime (thus void radius) is ruled by the surface tension. 
At lower temperatures this expansion by molecule displacement is not possible due to high viscosity of the medium. Liquid-like behaviour occurs when the relaxation time becomes comparable or shorter than the o-Ps lifetime. It is interesting to note that the effective viscosity in the scale of Ps bubble found by Winberg et al. was smaller by several orders of magnitude than the macroscopic one. The macroscopic viscosity is related to the medium as a whole that is composed of very large entangled molecules, while the Ps atom interacts with a small fragment of the polymer chain, and in this case the viscosity is like for a monomer multiplied by the number of chain elements involved in displacement; this number is very low (this kind of behaviour we have observed in the case of long chain alkanes, where the size of Ps bubble practically does not depend on the chain length).

Assuming the void shapes as spherical ("equivalent spherical"), one can use the Tao-Eldrup equation to obtain the free volume hole distribution $W(R)=s(\lambda) d \lambda / d R$. However, one has to remember, that the function $W(R)$ above is the product of a real $R$ distribution $V(R)$ and trapping probability $K(R)$. For spherical voids [153] :

$$
V(R)=\lambda_{b} \Delta \frac{1-\cos \left(\frac{2 \pi R}{R+\Delta}\right)}{(R+\Delta)^{2}} \frac{s(\lambda)}{K(R)}
$$

The trapping probability $K(R)$ is zero below $R_{\min }$. Hence, the positron methods do not bring information about those smallest voids. For larger radii Deng and Jean [153] postulate, in analogy to the trapping of free positrons [154], the linear dependence of trapping rate on the trap radius

$$
K(R)=1+a R
$$

$\left(a=80 \mathrm{~nm}^{-1}\right)$. Eq. 55 represents rather the first two terms in expansion of $K(R)$ into a series, whereas for large $R$ the trapping rate cannot rise to infinity.

In Eq. 40 it is assumed that the positronium atom once trapped cannot escape to another void or to the bulk. Yu et al. [155] have calculated the tunnelling rate between the voids and found it sufficiently large that Ps could sample many voids during its lifetime. However, Yu's results were found inconsistent with other data, particularly concerning Ps diffusion in polymers [156]. Calculations of the Ps transfer rate from one potential well to another by Baugher et al.[157] have shown that the rate given by $\mathrm{Yu}$ et al. is valid only for the wells of equal diameter, i.e. when the energies of the Ps states in both wells are identical, but even a slight 
difference in radii cuts off the tunnelling; in the case of polymers with a wide variety of void sizes and shapes, tunnelling seems to be of minor importance. On the other hand, one cannot neglect entirely the transitions from one void to another, keeping in mind how easily Ps transfers from small to large voids in plastic crystals [72]. In larger voids the zero-point energy is smaller, thus these voids are for Ps energetically favourable.

If the total free volume fraction $h$ is known, one can determine the number of free volumes per volume (or mass) unit:

$$
N_{h}=h / V_{h}
$$

where $V_{h}$ is an average void volume, $V_{h}=4 \pi R^{3} / 3$. In a classic way one can estimate the fraction $h$ from the measurement of sample volume as a function of temperature and pressure (PVT method) and applying the Simha-Somcynsky statistical equation of state [158]. An example of this approach is the study by Dlubek et al. [159] of CYTOP, a heterocyclicring-containing fluoropolymer. The PALS measurements of o-Ps lifetime and $h$ were performed as a function of temperature and pressure. The result for $N_{\mathrm{h}}$ is shown in Fig.58. With increasing temperature the void density decreases slightly, but it rises with pressure; at $200 \mathrm{MPa}$ almost twice, while their sizes are reduced. This can be explained by an assumption that the voids are not spherical, but well elongated and application of pressure divides them into fragments.

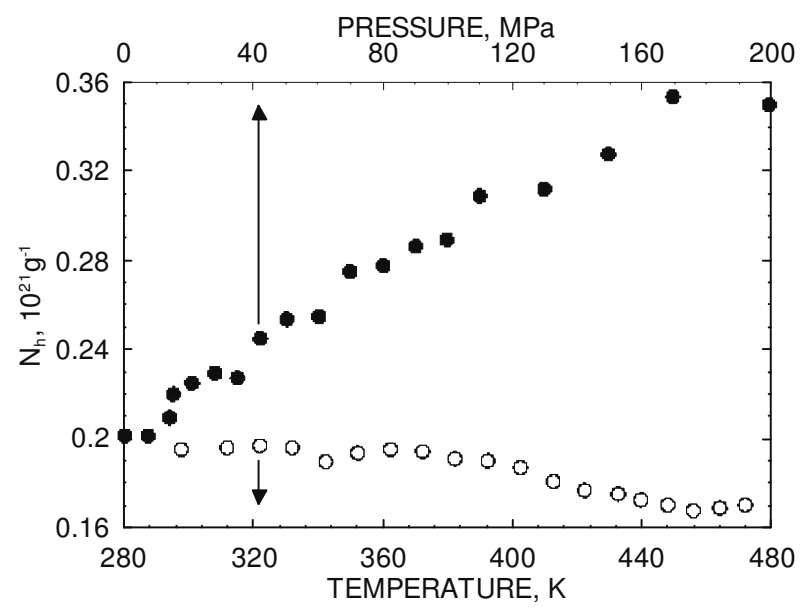

Fig. 58. Void density in CYTOP polymer as a function of temperature (open circles) and pressure (full circles). From M. Śniegocka, Ph.D. thesis, UMCS Lublin 2008 (and [159]). 
When only PALS data are available, Kobayashi et al. [160] proposed an empirical equation for the free volume fraction:

$$
h=\mathrm{C}_{3} V_{h}
$$

where $I_{3}$ means long-lived component intensity. Thus $\mathrm{C}_{3}$ means simply the number of voids per volume unit. The value of constant $\mathrm{C}$ is usually obtained by fitting the PALS data at the glass transition point $T_{g}$ to the $h$ estimated by the PVT method. For polyvinylacetate Kobayashi et al. give $\mathrm{C}=3.08 \mathrm{~nm}^{-3}$, for polymethylmetacrylate (PMMA) Maurer and Schmidt [161] propose $\mathrm{C}=2.28 \mathrm{~nm}^{-3}$. The idea of $I_{3}$ proportionality to the number of traps is still often used, however, it can be acceptable when that concentration is low (see mixed crystals [97], or vacancy component in plastic crystals [72]). The study by Maurer and Schmidt [161] shows that while above $T_{g}$ the equation (57) gives approximately the same slope $h(T)$ as the PVT method, below $T_{g}$ the temperature dependence is much better described by the relation:

$$
h=B_{0}+B_{1} V_{h}
$$

the intensity $I_{3}$ does not appear in this version at all. The gas diffusion measurements by Okamoto et al. [162] show also a better correlation of diffusion characteristics with hole size $V_{h}$, than with the product $I_{3} V_{h}$.

Many polymers are not entirely amorphous, their structure can be semi-crystalline (e.g. polyethylene, polystyrene). The structures can be very complex and depending on sample preparation. For example, polystyrene can contain crystalline fragments of four different phases, in two of them the chains are arranged in all-trans conformation, two other form helical structures trans-trans-gauche-gauche [163]; there are phase transitions between various structures. The crystallites have no well defined boundaries separating them from amorphous neighbourhood.

The measurements by Nakanishi et al.[164] have shown that the o-Ps intensity $\mathrm{I}_{3}$ changes linearly with the degree of crystallinity in poly(etherether-ketone), a similar result was obtained by Lind et al. [165] in polypropylene. This can be an indication that in the crystalline part positronium does not form; it locates in the amorphous part only. If so, one can expect a proportionality of $h$ to $I_{3}$ as a measure of crystallinity degree. The supposition that Ps cannot be formed in the crystalline phase is an excessive simplification. In the papers cited above the spectra were decomposed into three exponentials (one only for o-Ps). In the fourcomponent fit one can try to ascribe the shorter of the o-Ps components to the crystalline phase, the longer - to the amorphous one. Serna et al.[166] 
estimated the probabilities of Ps formation as 0.15 and 0.43 in the crystalline and amorphous regions of polyethylene, respectively.

However, the o-Ps spectrum composed of two peaks is observed also in entirely amorphous structures. In this case one can suppose that two groups of cavities are present, or the shorter-lived component belongs to the channels interconnecting larger cavities

\subsection{Applications}

The PALS technique is particularly useful as a tool of controlling the modification of polymer structure during technological processes. As an example one can mention the positronium study of gas permeation through the polymer, which is in large extent determined by the sizes and density of the free volume holes. For example, the PALS method was used in the tests of contact lenses which should be permeable for oxygen and nitrogen [167] .

Glassy polymer membranes are used for gas separation, e.g. $\mathrm{H}_{2}$ from hydrocarbons, alkanes from olefins, water from isopropanol etc. The dimensions of holes, determining the permeability can be modified by various kinds of treatment. The sizes of individual free volumes can be reduced by application of high pressure up to $200 \mathrm{MPa}$ when the polymer is in liquid-like state and then decreasing the temperature. The increase of the free volume holes can be made by exposing the sample to a gas atmosphere of several $\mathrm{MPa}$ at room temperature and then decreasing the pressure at very low rate (rapid depressurization leads to another interesting effect of polymer foaming). Particularly effective from this point of view is the sorption of $\mathrm{CO}_{2}$, swelling and plasticizing the polymer [168-171]. If a polymer is in its rubbery state, at application of high pressure of $\mathrm{CO}_{2}$ the lifetime and intensity rise, but after gas evacuation these parameters return to their initial values. If the polymer is glassy, the increased voids remain also after the gas removal. Swollen structure is out of equilibrium, but the relaxation times can be extremely long. In poly[1-trimethylsilyl]-1-propyne (PTMST) swelling by supercritical $\mathrm{CO}_{2}$ increases the radii of free volumes to $0.7 \mathrm{~nm}$ (respective o-Ps lifetime is $9.2 \mathrm{~ns}$ !) and the relaxation time is estimated as about 30 years [171].

Swelling shifts the glass transition point down. The polymer treatment by gas sorption can introduce also other effects; supercritical $\mathrm{CO}_{2}$ induces crystallization of semi-crystalline polymers. 
The PAL spectroscopy is useful also in the study of polymer degradation from the viewpoint of two contradictory aspects: rapid degradation of polymer waste is interesting in the environement protection, slowing down this process is important when the durability of product (construction elements, protective coatings) is needed.

Oxygen plays a main role in the degradation of materials. Ito et al.[172] observed the effect of oxydation in pure polyethylene exposed to air in $373 \mathrm{~K}$. The reaction of oxygen with thermally produced radicals create the carbonyl groups. Due to the affinity of positrons to oxygencontaining polar groups the formation of positronium is reduced. The decrease of the o-Ps component intensity with exposure time is visible in PALS spectra; the $S$ parameter in DBARL decreases too. The samples of polyethylene containing antioxidant (octadecyl 3-(3',5'-di-tert-butyl4'hydroxyphenyl) propionate do not show any change of $\mathrm{I}_{3}$ and $\mathrm{S}$ during 30 days. Even in natural conditions (air atmosphere, room temperature) the effect of degradation of polyethylene is well visible in the scale of several months, as can be seen in Fig. 59 [173].

The effects of degradation are important in the case of films, membranes, coatings, i.e. the objects of small thickness, thus the use of positron beams of controlled energy is the most appropriate; it is described in Sec.11.

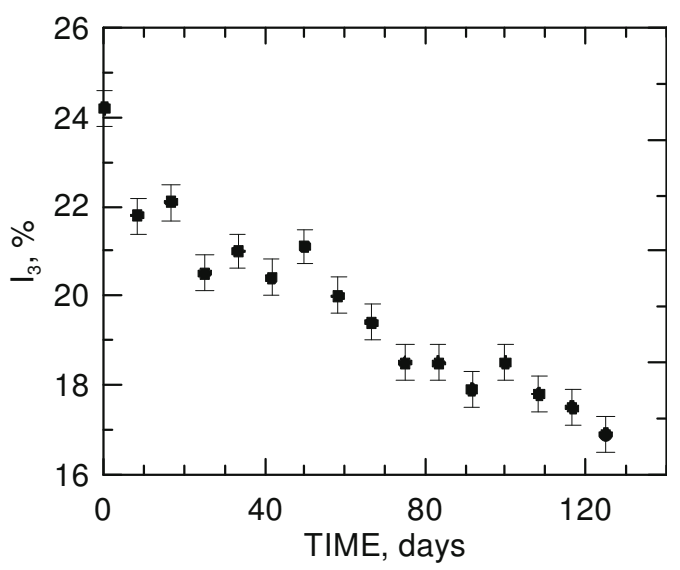

Fig. 59. Ageing of polyethylene. Decrease of the o-Ps intensity with time. Courtesy of W. Osoba.

Commercially available polymers contain various additives (like antioxidants mentioned above), which vary from one producer to another. Such additives, usually unknown to the user, can be also electron or hole scavengers and bring further complication to the interpretation of the positronium intensity. The list of factors influencing $I_{3}$ can go on and on. Generally, the intensity of long lived component is a result of a great 
number of processes, material properties, external influences, thus, the assumption that it can be a measure of the number of free volume holes only is a simplification

The presence of sufficiently large free volume is necessary but not sufficient to bind an $\mathrm{e}^{+} \mathrm{e}^{-}$pair into a Ps atom. If e.g. the affinity of electron (or positron) to the molecules of the medium is large, it can be energetically favourable to not form the Ps bound system. As it was shown by Ito et al.[174] the high electron affinity of pyromellitic dianhydride causes that polyimides containing this radical show no positronium formation (to this group of polyimides belongs the popular Kapton).

\section{THE ORTHO-PARA CONVERSION}

The lifetime of o-Ps in condensed matter is determined mainly by the pick-off process. In the case of free volumes in crystalline solids or polymers three quantum annihilation can be neglected, as introducing the correction of the order of $1 \%$. However, when the pick-off rate is small, it has to be taken into account. Such a situation can appear in media with relatively large free volumes, like porous materials; this problem will be discussed later, in Sec.10.

Among other processes influencing the o-Ps lifetime, the most common is ortho-para conversion due to the interaction of Ps with paramagnetic molecules. In particular, such a process occurs in the presence of oxygen. The ground state of $\mathrm{O}_{2}$ molecule is a triplet state, the collisions of o-Ps with $\mathrm{O}_{2}$ lead to spin exchange; after the conversion of o-Ps into p-Ps rapid two-quantum decay occurs.

This effect is best pronounced in two cases: in liquids (due to dissolved oxygen) and in porous membranes permeable for gases and adsorbing oxygen molecules [175]. In the last case the effect of conversion is strong when polymers contain large voids, like poly[1tromethylsilyl]-propine (PTMST). The o-Ps lifetime in this polymer shows two components, the longer-lived of them exceeds $10 \mathrm{~ns}$; respective free volumes $(\sim 0.7 \mathrm{~nm})$ assure high permeability of this medium for gases. Fig. 60 shows the lifetime in PTMST as a function of gas pressure [176]. Oxygen pressure of 1 bar reduces the lifetime from $13 \mathrm{~ns}$ to $2.5 \mathrm{~ns}$, while non-paramagnetic nitrogen has practically no influence on the o-Ps lifetime. 


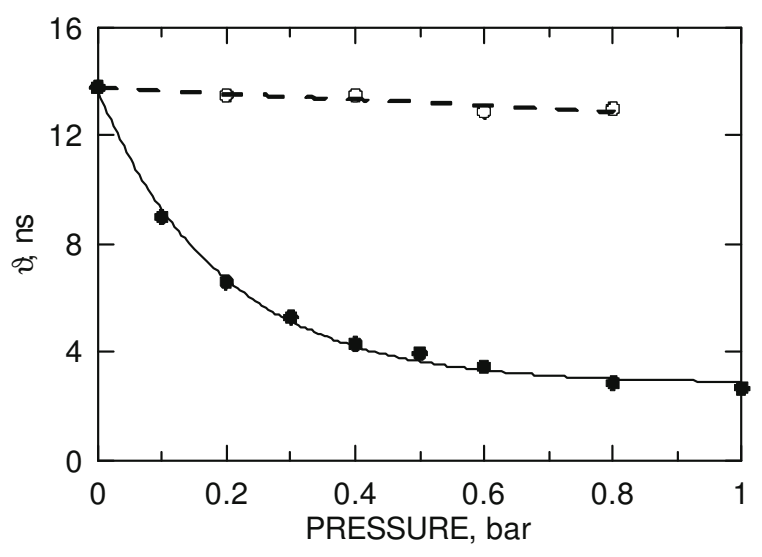

Fig. 60. The longest-lived component in the PALS spectrum of PTMST as a function of pressure: dots - oxygen, open circles - nitrogen [176].

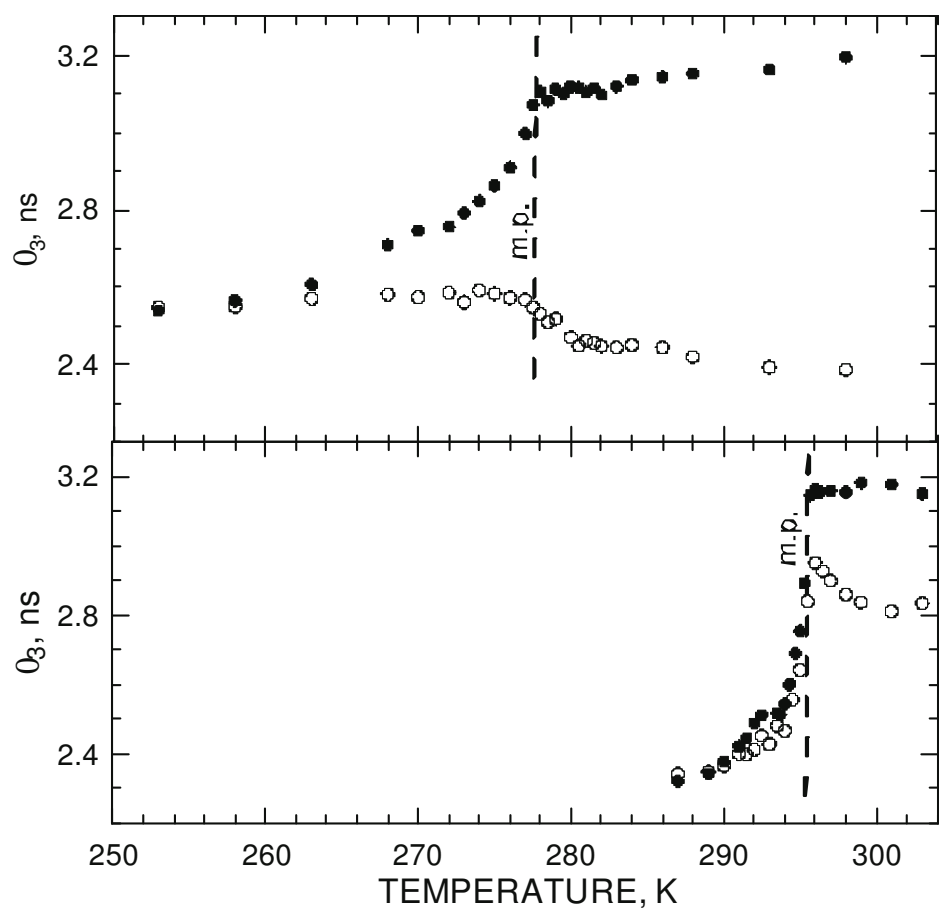

Fig. 61. Temperature dependence of the o-Ps lifetime in: top - cyclohexane [69] and bottom - n-heptadecane (B. Zgardzinska, unpublished). Dots sample isolated from oxygen, empty circles - the sample in atmospheric air. Melting points indicated by vertical dashed lines.

The influence of oxygen on the o-Ps lifetime in liquids is demonstrated in Fig. 61 showing the o-Ps lifetime in cyclohexane and a representative of $n$-alkanes, $n$-heptadecane, as a function of temperature in two cases: 
- the sample was degassed and over it the own saturation vapour only was present;

- the sample was in contact with the air at normal pressure.

It is seen that in liquid cyclohexane contacting with the air the o-Ps lifetime is shortened by about $0.8 \mathrm{~ns}$, moreover, the lifetime shortening is observed even in the plastic solid phase (see Sec. 6.4.1) down to $10 \mathrm{~K}$ below the melting point, indicating the easy penetration of oxygen also into the disordered solid. In the rotator phase the effect of oxygen penetration is not observed; the o-Ps lifetime begins to shorten above the melting point only. The scale of lifetime reduction by ortho-para conversion in alkanes, both even-numbered (C28) and odd- ones (C17), is much smaller than that found in cyclohexane, about $0.3 \mathrm{~ns}$.

\section{POSITRONIUM FORMATION ON TRAPPED EXCESS ELECTRONS. IRRADIATION EFFECTS}

Still in 80's it was found that after cooling from room temperature to that of liquid nitrogen or similar, the o-Ps intensity in polymers increases gradually with time [177-179]. Fig. 62 shows the rise of o-Ps intensity in polypropylene, after cooling from room temperature [180].

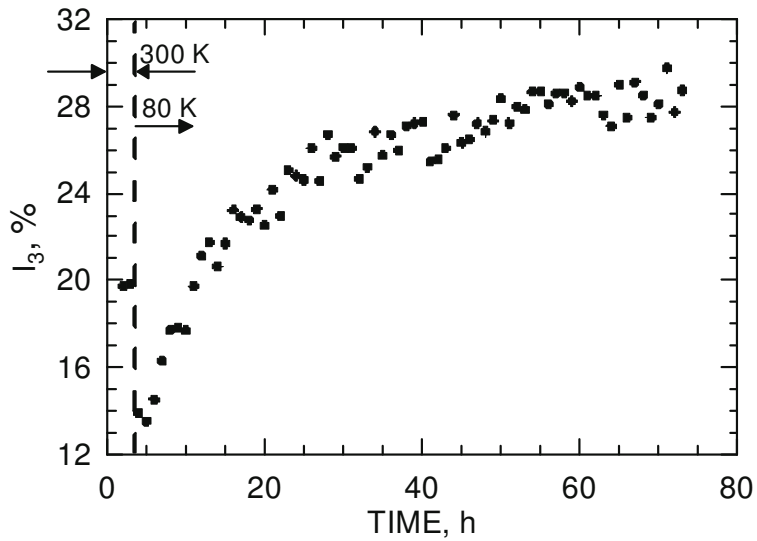

Fig. 62. The rise of the o-Ps intensity with time in polypropylene after cooling from $300 \mathrm{~K}$ to $80 \mathrm{~K}[180]$.

At $80 \mathrm{~K}$ an increase of $I_{3}$ more than twice compared to the initial value is observed after reaching the saturation state. The effect of $I_{3}$ growth with time was seen also in some crystalline organic solids, like cyclohexane [79], long chain alkanes [181] etc. During the growth of the o-Ps intensity no changes of the lifetime were observed. Wang et al. [182] proposed to explain the rise of the o-Ps intensity as induced by irradiation 
products. High energy positrons in ionization acts produce excess electrons and some of them are trapped in the polymer structure. At low temperatures the molecular motions are frozen and a trapped electron can survive in such a trap for very long time. Since the moment of cooling the sample to the temperatures below about $150 \mathrm{~K}$, continuous irradiation by positrons from the positron source initiates an accumulation of trapped electrons. With the elapse of time the subsequent positrons injected into the sample meet more and more trapped electrons and the probability of binding with them into Ps atom rises. The o-Ps intensity growth is thus determined by the dose of positrons absorbed in the sample. The proportionality of o-Ps increase to the density of localized electrons was clearly demonstrated by Hirade et al. [183]. An external source of $\gamma$-rays irradiated the poly-methylmetacrylate (PMMA) sample in which the PALS spectrum was measured; the supplied dose rate was several $\mathrm{kGy} / \mathrm{h}$ (in this case the dose from positron source was negligible compared to the external gamma source). The density of trapped electrons was determined by electron spin resonance (ESR) measurements. In polyethylene the rise of $\mathrm{o}-\mathrm{Ps}$ intensity by $1 \%$ corresponds to an accumulation of $4 \cdot 10^{14}$ electrons per gram.

A further validity test of this mechanism of the o-Ps intensity growth is demonstrated by sample illumination. The depth of electron traps is estimated as (0.5-3.0) eV, thus visible light should be sufficient to make the traps empty. After illumination the o-Ps intensity returns to the initial low value, like for non-irradiated sample. The experiments of this kind were performed by Hirade et al. [183, 184], Ito et al. [185] with polymer samples, and followed by other authors applying the light to throw electrons out the traps in alkanes [186, 187]. Fig. 63 shows the intensity of the o-Ps component in $\mathrm{C} 17 \mathrm{n}$-alkane as a function of time at $200 \mathrm{~K}$, the sample was irradiated by a positron source of $0.3 \mathrm{MBq}$. After about $20 \mathrm{~h}$ the intensity reached a maximum - twice of that at the beginning of treatment. At continuation of measurement the intensity $\mathrm{I}_{3}$ began to decrease slowly. The measurements were repeated and after $68 \mathrm{~h}$ the sample was illuminated by LED diodes of wavelength at the maximum $0.95 \mu \mathrm{m}$ (near infrared) which restored the initial value of intensity - all excess electrons were thrown out the traps. The depth of traps in alkanes seems to depend on the chain length. 


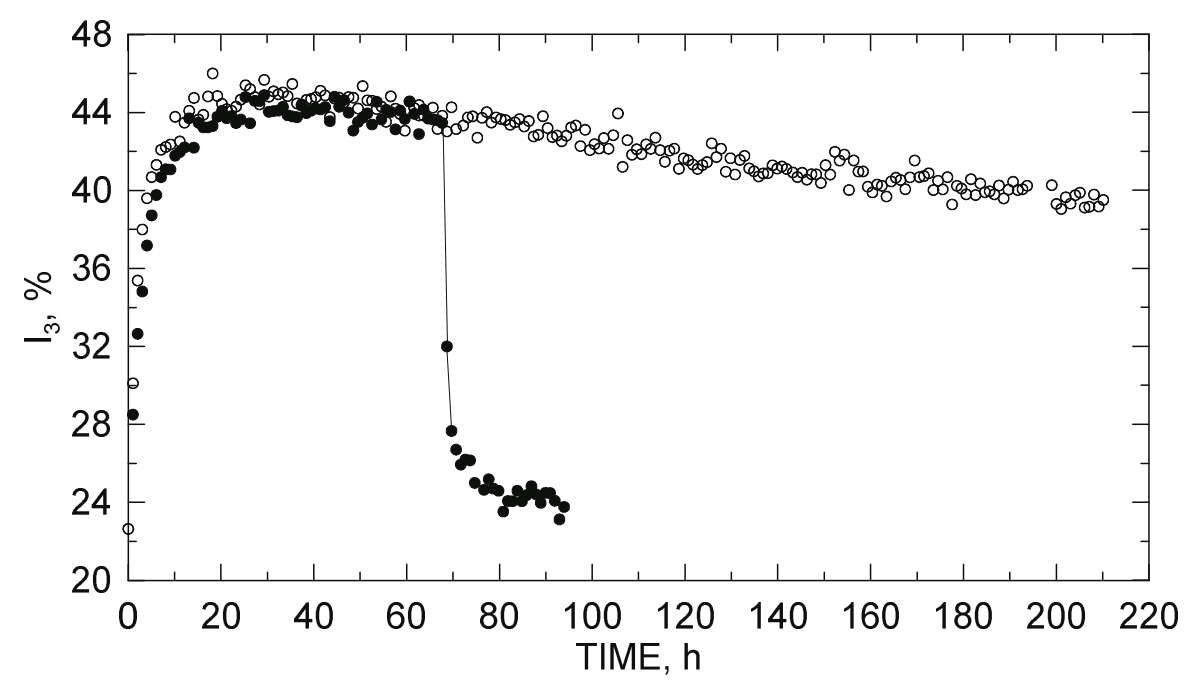

Fig. 63. The o-Ps intensity in n-heptadecane $\mathrm{C} 17$ as a function of irradiation time. Open circles - in darkness, full circles $-68 \mathrm{~h}$ in darkness and then illuminated by $0.95 \mu \mathrm{m}$ LED. Temperature 200 K.[187]

In $\mathrm{C} 17$ the photon energy of $1.3 \mathrm{eV}$ was sufficient to empty the traps, while in n-hexane C6 Shantarovich et al [188] have found that the visible light does not remove all trapped electrons. The dependence of the $I_{3}$ intensity in high density polyethylene HDPE on the wavelength of illumination was investigated by Nahid et al. [189] using optical high pass filters. Pietrow and Wawryszczuk [190] observed that at illumination of n-heptane C7 by LEDs of various wavelengths. The reduction of the $I_{3}$ intensity begins already at $2000 \mathrm{~nm}$, full emptying the traps needs the light of $\lambda<500 \mathrm{~nm}$ (Fig.64). Analogous measurements for cyclohexane one can find in Ref. 66.

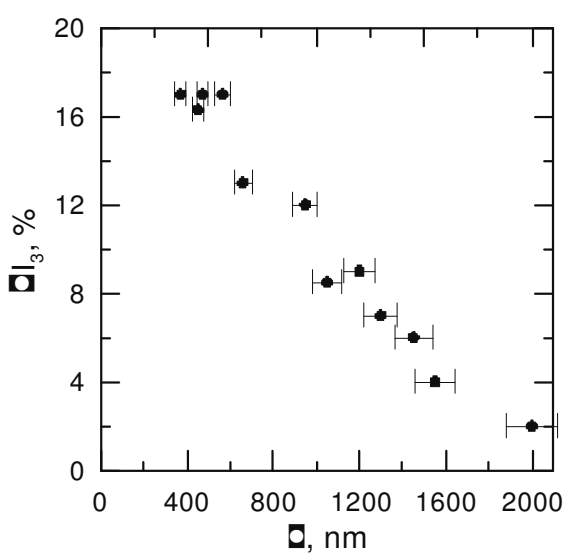

Fig. 64. Reduction of o-Ps intensity in n-heptane C7 after illumination by LEDs as a function of light wavelength [190]. Temperature $133 \mathrm{~K}$. 
Effectiveness of electron trapping depends on temperature as can be seen in the case of n-heptadecane C17 [187]. The sample was cooled to $130 \mathrm{~K}$ and stored for $20 \mathrm{~h}$ to reach the maximum of $I_{3}$ and then the temperature was increased. Up to about $190 \mathrm{~K}$ the intensity of o-Ps increased further, and then decreased rapidly due to thermal collapse of the traps destroyed by activation of intramolecular motions (Fig. 65). At room temperature electron trapping is not observed.

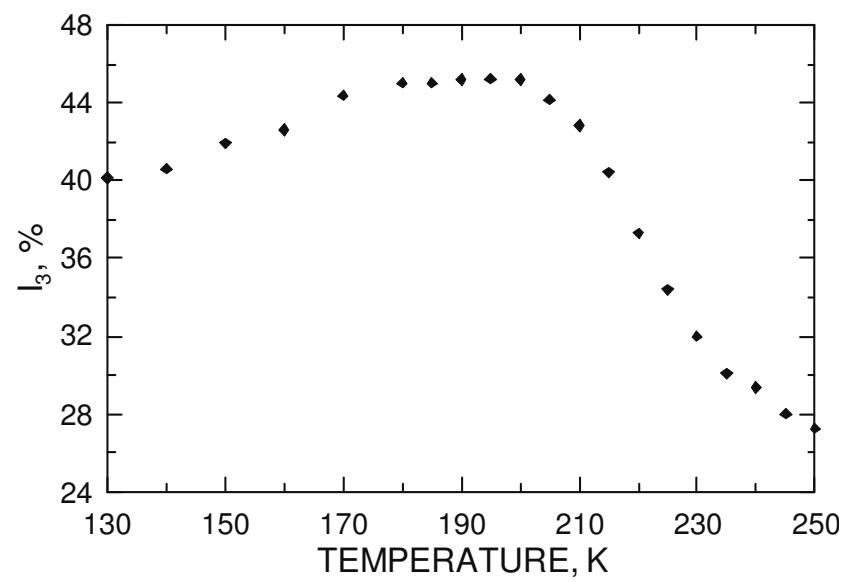

Fig. 65. The o-Ps intensity in n-hepta-ecane $\mathrm{C} 17$ as a function of rising temperature after reaching satura-ion at $130 \mathrm{~K}$ [187]. Measurements in darkness.

The rise of $I_{3}$ between $130 \mathrm{~K}$ and $190 \mathrm{~K}$ observed in n-heptadecane (Fig. 65) and other alkanes, can result from either an increase of trapped electron density or greater positron mobility facilitating its migration to the traps. In order to check the mechanism of $I_{3}$ rise with temperature, the sample of heptadecane was kept at $130 \mathrm{~K}$ up to reaching the saturation of $I_{3}$ and then the temperature was increased stepwise to $185 \mathrm{~K}$ (Fig. 66); the o-Ps intensity rose immediately to the value corresponding to that temperature like in Fig.65. After $10 \mathrm{~h}$ storage, the temperature returned to $130 \mathrm{~K}$ and the o-Ps intensity returned too [191].

The time constant of electron accumulation was about $8 \mathrm{~h}$, while the time needed to change the temperature by $55 \mathrm{~K}$ was less than $15 \mathrm{~min}$, (the time of spectrum accumulation directly after the change of temperature was reduced to 15 min too), thus the density of trapped electrons could not change substantially and the increase of $I_{3}$ is due entirely to increased mobility of positrons. 


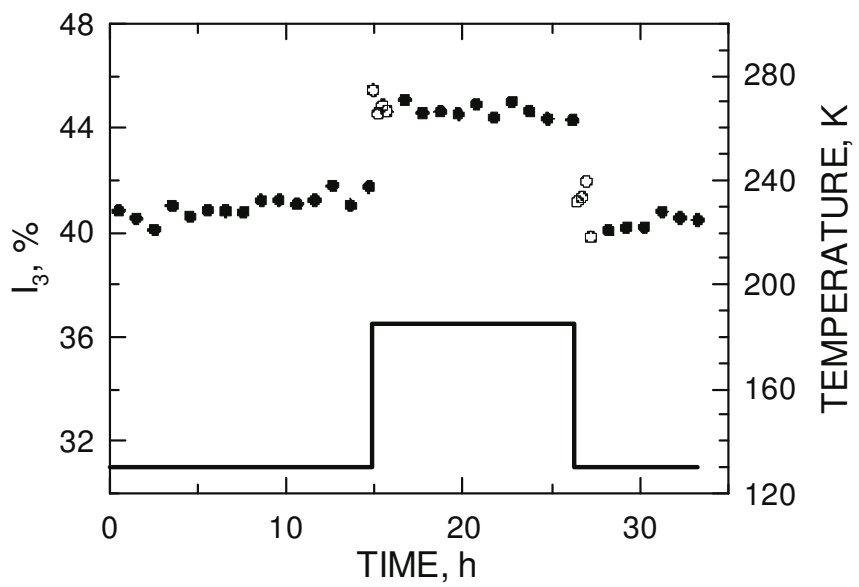

Fig. 66. Changes of o-Ps intensity in n-hepta-decane at stepwise changes of temperature after preliminary stor-age to saturation at $130 \mathrm{~K}$. Open symbols are for the time of spectrum collection reduced to $15 \mathrm{~min}$. From [191].

With prolonged irradiation of the sample, a slow decrease of $I_{3}$ intensity appears, as it is seen in Fig.63 The observed intensity is the result of two competing processes with participation of excess electrons: trapping and combining with radicals, which also accumulate as a consequence of irradiation [192]. At room temperature some radicals are still long-lived, thus the decrease of $I_{3}$ is not shadowed by trapping effect and can be observed, in particular in many polymers. With further increase of temperature the effect of radicals disappears too [193].

When the annihilation of o-Ps in a polymer needs two components to describe the PALS spectrum, only the longest of them $\left(\tau_{4}, I_{4}\right)$ changes with time, the shorter one is stable.

An interesting systematic study of Ps quenching by radicals was presented by Dlubek et al.[194] in the case of a series of poly( $\alpha$-olefins). These linear polymers were synthetized as containing the side branches of the length from 3 to 20 methylene groups. The decrease of $I_{4}$ intensity at $\mathrm{e}^{+}$irradiation (time scale of the order of $2 \mathrm{~h}$ only) was strongest for propylene side branch, then diminished systematically with lengthening the branch and for $\mathrm{n}=18$ and 20 the decrease was substituted by $I_{4}$ rise (measurements at room temperature),

The number of trapped electrons per unit dose can lie in very broad limits for various polymers, e.g. $I_{3}$ in PMMA is much less sensitive to irradiation than in polyethylene [183]. 
In the crystalline hydrocarbons the effect of electron accumulation appears in the low temperature "rigid" phase only. No such an effect in partly disordered phases: rotator phase of alkanes and plastic phase of cyclohexane. Also in the case of multicomponent mixed alkanes the disordering of structure introduced by various molecule lengths reduces the electron trapping effect. For an average length $\bar{n}=19$, at the width of molecule length distrubution $\Delta n=\left(\sum c_{n}(n-\bar{n})^{2}\right)^{1 / 2}$ equal 2 , the trapping effect comes to zero [195]. Accumulation of excess electrons is not observed also in n-alkanols-1.

\section{EXTENSIONS OF TAO-ELDRUP MODEL. POROUS MEDIA}

\subsection{The role of excited states.}

Already at early stage of positron spectroscopy it was noticed that the lifetimes measured for large voids do not fit the simple Tao-Eldrup predictions. One of attempts to improve the consistency of the model and experiment was done by Shantarovich et al. [196], modifying the shape of potential well (rectangular, but deepened near the walls). More realistic approach consists in taking into account the population by Ps the energy levels in the potential well, lying higher than the ground level [197, 198]. With the increase of free volume radii, besides the zero-point level, also other levels begin to appear and in favourable conditions become populated. One can easily extend the Eq.30 for cuboid to describe the o-Ps annihilation rate from individual state $\left(n_{1}, n_{2}, n_{3}\right)$ for arbitrary number of halfwaves $n_{\mathrm{i}}$ between the walls:

$$
\lambda_{p o}^{n_{1} n_{2} n_{3}}=\lambda_{b}\left[1-\prod_{i=1}^{3}\left(\frac{a_{i}}{a_{i}+2 \Delta}+\frac{(-1)^{n_{i}+1}}{n_{i} \pi} \sin \frac{n_{i} \pi a_{i}}{a_{i}+2 \Delta}\right)\right]
$$

(Eq. 59 is equivalent to a similar equation given by Gidley et al.[199], where $\left.x_{i}=a_{i}+2 \Delta\right)$. In porosimetry the rectangular geometry is rarely used, but it is easier in calculations (sine wavefunctions instead of more complex ones).

In a spherical well the levels with non-zero angular momentum $l$ begin to appear for the radii $R>\pi \hbar / 2\left(m_{e} V\right)^{1 / 2}$. The wavefunctions for the states with momentum $l$ are Bessel ones $j_{l}(r)$. Thus, maintaining the Tao-Eldrup concept of broadened infinitely deep well and spherical geometry, we can write [198]: 


$$
\lambda_{p o}^{n l}=\lambda_{b} \int_{X_{n l} R /\left(R+\Delta^{\prime}\right)}^{X_{n l}} j_{l}^{2}(r) r^{2} d r / \int_{0}^{X_{n l}} j_{l}^{2}(r) r^{2} d r
$$

where $X_{n l}$ is the $n$th node (zero crossing point) of Bessel $j_{l}(r)$ function. Broadening of the well is written here as $\Delta^{\prime}$. For the ground state $\Delta^{\prime}=\Delta$ because the wavefunction $j_{0}(r)$ is the same as $\sin r / r$ appearing in the Tao-Eldrup model, but for other states it can have other values (the same concerns the rectangular voids, Eq. 59). Fig. 67 shows the values of the lifetime to annihilation $\tau_{n l}=1 /\left(\lambda_{p o}^{n l}+\lambda_{T}\right)$ for several low lying levels in a spherical well assuming $\Delta^{\prime}=\Delta$.

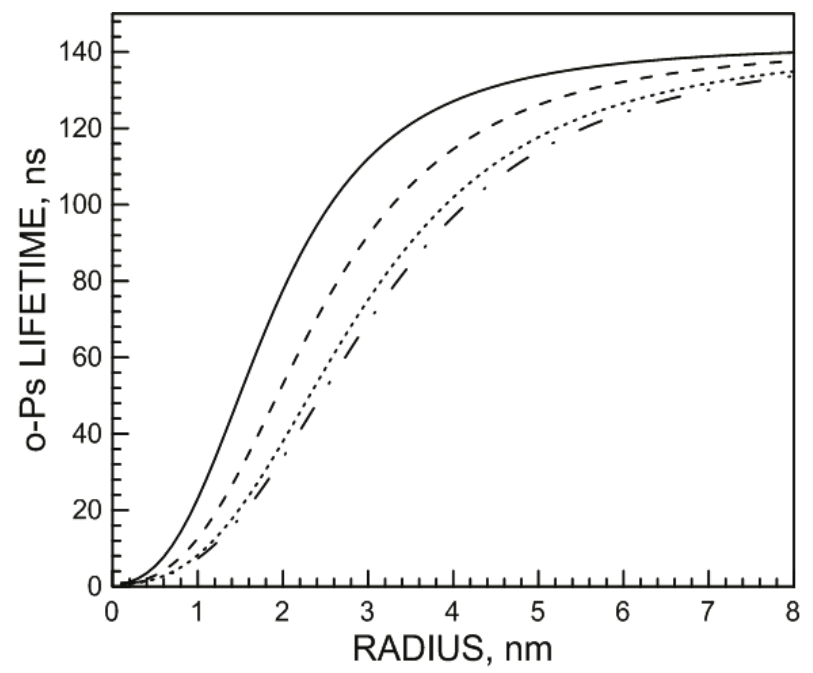

Fig. 67. The o-Ps lifetime to annihilation from lowest energy levels in the rectangular potential well (spherical voids): 1s (solid line), 1p (dashes), $1 \mathrm{~d}$ (dots), 2s (dash-dot)

As long as the spacings of Ps levels in the well are much larger than $k T$, the equilibrium population of the levels above the lowest one is negligible and simple Eq. 29 is still valid. The spacings of the levels decrease with the void radius like $1 / R^{2}$, and at room temperature, for $R$ exceeding $1 \mathrm{~nm}$, the population of upper levels has to be accounted. The shape of the o-Ps lifetime spectrum depends now on the population of various levels.

For the states other than the lowest one we distinguish two lifetimes: the mean time to annihilation $\tau_{\mathrm{nl}}$ and the time of residence in the state $(n l)$ - let call it "dwell time" $\tau_{D}$. At the moment of falling into the void the Ps energy corresponds to the upper rim of the potential well and, to be 
trapped, Ps needs to lose at least a part of the energy by interaction with the surrounding bulk. Further acts of interaction (excitation and absorption of phonons, excitation of molecular vibrations etc.) lead to thermalization of positronium. If the equilibrium has not been achieved yet, the high-lying states are over-populated. Reaching equilibrium means that Ps changes its state many times before annihilation, $\tau_{D} \ll \tau_{n l}$.

If the $R$ value is discrete (or the width of the $R$ distribution is very narrow) one can state that positronium is thermalized, when the decay rate calculated by fitting the exponential to the tail of experimental distribution stops to depend on the delay above which it is observed (i.e. the measured o-Ps lifetime becomes independent from the choice of the initial point in the analysis of the long-lived part of the spectrum). The experimental data for ultrafine silica powders give the time to full thermalization rather long: $10 \mathrm{~ns}$ [200], $20 \mathrm{~ns}$ [201]; in the case of aerosil [202] up to $50 \mathrm{~ns}$.

If there is no equilibrium, in an extreme case when $\tau_{\mathrm{D}} \gg \tau_{\mathrm{nl}}$, one should see a separate exponential component for each of the involved levels.

In the state of equilibrium a Boltzmann-like distribution can be assumed, and for given $R$ one should expect one exponential component with average pick-off rate $\lambda_{p o}$ :

$$
\lambda_{p o}=\frac{\sum_{q} \lambda_{q} g_{q} \exp \left(-E_{q} / k T\right)}{\sum_{q} g_{q} \exp \left(-E_{q} / k T\right)}
$$

where $g_{q}$ is the statistical weight of the $q$ th state, $E_{q}$ - its energy. The energies of states enter Eq. 61, thus their values are important for final result. In the model proposed in [136], where the potential well of finite depth is substituted by infinite one broadened by $\eta$, they should be:

$$
E_{q}=\frac{\hbar^{2}}{4 m_{o}} \frac{X_{n l}^{2}}{(R+\eta)^{2}}=1.90 \times 10^{-2} \frac{X_{n l}^{2}}{(R+\eta)^{2}}
$$

for $E_{q}$ in $\mathrm{eV}$ and $R$ in $\mathrm{nm}$. The energies calculated for finite well and infinite one as a function of $R$ are shown in Fig. 49. The $\eta$ parameter diminishes with deepening the potential well. For the case of silica the TOF measurements [62] give $U \approx 3 \mathrm{eV}$; thus for the ground state in the well $\eta$ should be $0.084 \mathrm{~nm}$. In Fig. 68 the $\tau_{3}(R)$ dependences calculated by accounting various numbers of levels are shown, assuming the equilibrium population at room temperature. With lowering temperature the relation o-Ps lifetime vs. pore radius tend to the Tao-Eldrup model. 


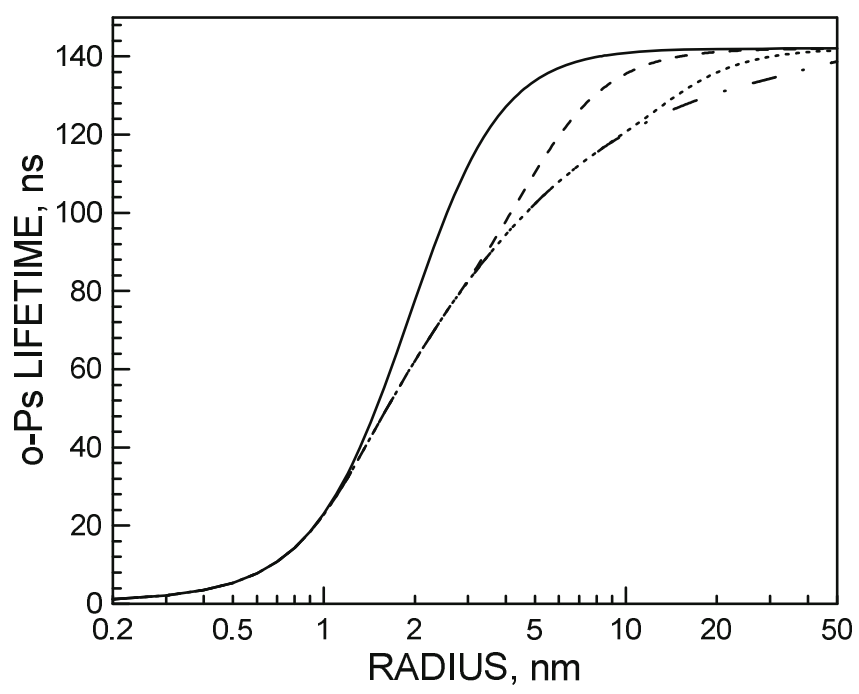

Fig. 68. Relation o-Ps lifetime vs. spherical pore radius, calculations included: 1 (TE model, solid line), 10 (dashed), 100 (dotted), 1000 (dash-dot) energy levels Parameters $\Delta$ and $\eta$ assumed equal to $0.166 \mathrm{~nm}$ (calculations by R. Zaleski). Room temperature.

The model taking into account the population of higher located levels of Ps in the rectangular potential well we will call "Extended Tao-Eldrup model" (ETE).

As a rule, the higher is the level location, the shorter is the respective lifetime. With an increase of temperature the population of higher located levels rises. Direct consequence of the population of excited levels is the decrease of the o-Ps lifetime with rising temperature, as can be seen in Fig. 69 for porous Amberlite.

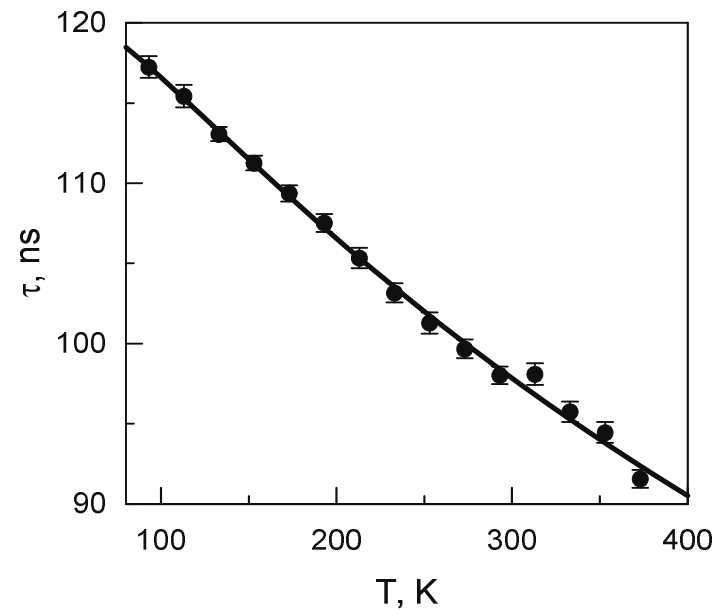

Fig. 69. The o-Ps lifetime in Amberlite XAD7HP as a function of temperature. The solid line is the result of calculation with Eq. 61, assuming pore radius of $2.65 \mathrm{~nm}$. Courtesy of R. Zaleski. 
Accounting the Ps annihilation from higher levels in the well [197], PALS and related techniques opened a new method of porosimetry.

Very often the pores have the form of capillaries (cylindrical channels). In the case of cylindrical geometry the radial wavefunctions are Bessel $J_{m}(r)$ ones and instead of Eq. 60 we receive [198]

$$
\lambda_{p o}^{n m}=\lambda_{b} \int_{Z_{n m} R /(R+\Delta)}^{Z_{n n}} J_{m}^{2}(r) r d r / \int_{0}^{Z_{n n}} J_{m}^{2}(r) r d r
$$

( $Z_{n m}$ are the nodes of $J_{m}$ function). The decay constants from Eq. 63 enter to the Eq.61. The motion along the infinite capillary axis is not quantized, thus the energies are:

$$
E_{n m}=\frac{\hbar^{2}}{4 m_{0}} \frac{Z_{n m}^{2}}{(R+\eta)^{2}}+E_{a x}
$$

The average energy of axial motion $E_{a x}$ should be $k T / 2$, the same for all states, thus in Eq. 61 it is cancelled, if one neglects the fact that it is an average energy only.

When the structure of pores is described as a bundle of capillaries, their average radius is $2 V / S$, where $V, S$ are total pore volume and surface area, respectively. Note that the $V / S$ ratio is the same for a capillary of either circular or square cross section when $a_{1}=a_{2}=2 R$, thus insensitive to the details of geometry. However, it is not valid for the PALS method; the o-Ps lifetime is not the same for the two discussed shapes. For moderate sizes of transverse section the lifetime of o-Ps can be by about $20 \%$ longer for a square section than for a circular one.

In Eq. 61 the expressions for $\lambda_{\mathrm{q}}$ and $E_{q}$ contain the constants $\eta$ and $\Delta$ introduced due to the calculation of both values using the Tao-Eldrup approach. Essentially these constants should be different for each energy level, but this fact will complicate the calculations to an entirely unpractical level. Thus, the question arises, if a universal value for all these parameters can be found, giving sufficiently good approximation. For the first time such an attempt was presented for porous Vycor glass of relatively narrow pores [204]; the value of $\Delta=\eta=(0,191 \pm 0.008) \mathrm{nm}$ was obtained. Gidley et al [199] have found for silica films the value 0.18 $\mathrm{nm}$. A precise test was performed by Kallmann et al. [205] for cylindrical pores etched in Vycor glass. For a rich set of radii they stated that quite good agreement of the data from the classic nitrogen adsorption method and PALS is obtained for $\Delta=\eta=0.193$ (Fig. 70). The version assuming an equilibrium population and an universal $\Delta$ parameter is now commonly 
used. The value of "universal" $\Delta$ given above is fitted to silica based materials and need not to be exactly correct for others, e.g. porous polymers.

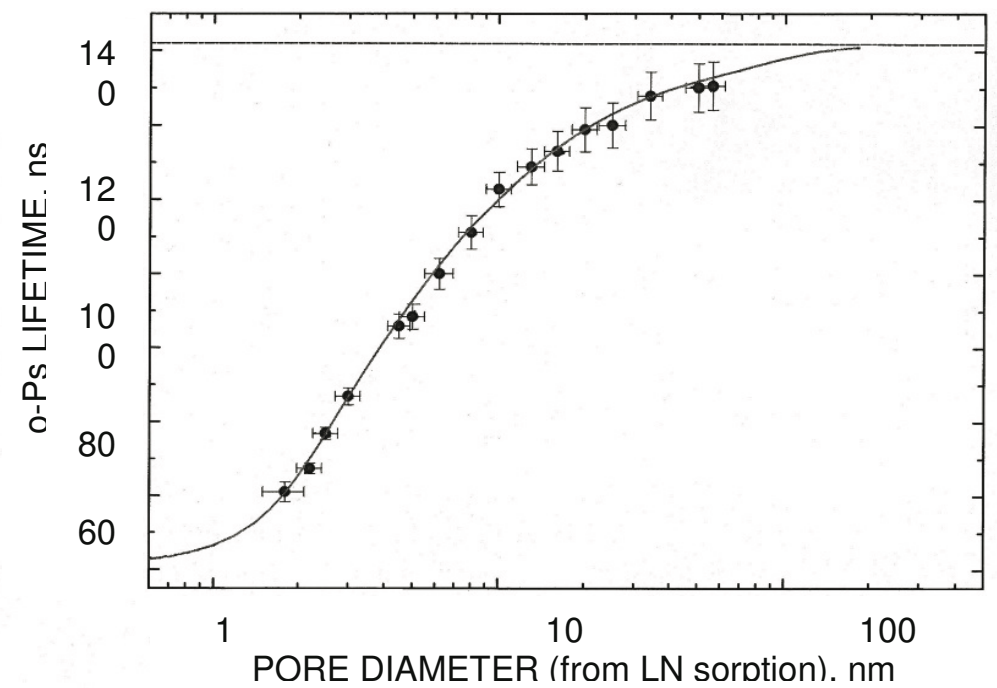

Fig. 70. The o-Ps lifetime in porous Vycor glass; the pore radii were determined by the liquid nitrogen adsorption. The solid line from the calculations using Eqs. 61, 63,64 for $\Delta^{\prime}=\eta=0.193 \mathrm{~nm}$. Room temperature. Courtesy of R. Krause-Rehberg.

The anihilation of o-Ps while cascading through higher energy levels makes the decay curve nonexponential with the initial slope more inclined. A semi-classic approach to this problem can be found in the paper by Dauwe et al. [206]: the decay rate is assumed proportional to the number of Ps collisions with the walls of void per time unit; the higher the energy - the more collisions per time unit, therefore faster decay (probability of annihilation per one collision assumed to be constant, and energy independent).

It is important to state that the positron methods can probe closed pores as well as open ones, while these first ones are not accessible for standard adsorption or intrusion methods. Classic silica gels and porous Vycor glasses belong to the materials with open pores. In such a case the length of a pore plays an important role: if the pore is short, o-Ps can diffuse out and escape into vacuum, and then its lifetime has little common with the pore diameter. According to the calculations by Eijt et al. [207] the escape of o-Ps is efficient when the distance from the Ps 
formation point to the outlet of pore is less than $1 \mu \mathrm{m}$. Fortunately, in the porous media like those mentioned above the pore length is much larger. Even at unfavourable etching conditions of Vycor glass: low temperature $323 \mathrm{~K}$, short time $20 \mathrm{~min}$, the thickness of etched layer (and then the pore length) was found equal about $24 \mu \mathrm{m}$ [208], i.e. a value much longer than the escape length.

During the diffusive walk along the pore the newly formed o-Ps thermalize and in the case of short pore channels is emitted into the vacuum with an energy distribution expected to be Maxwell distribution. However, the reduction of the particle energy in the pore is limited by the uncertainty principle, thus the thermalization at very low temperatures is possible only when the pore diameter is sufficiently large. Mariazzi et al. [209] have measured the velocity distribution of Ps escaping from the pores by TOF method. The samples were of silicon electrochemically etched to produce the pores of diameter from about $5 \mathrm{~nm}$ to $20 \mathrm{~nm}$. When positrons were implanted into the sample with the energy of $10 \mathrm{keV}$, the produced Ps needed about 10-20 ns to leave the pores; at the energy of 20 $\mathrm{keV}$ that time rises to about $100 \mathrm{~ns}$. The results obtained in this experiment (Fig. 71) indicate that o-Ps emitted from the pores contains two components, one of them corresponds to actual thermal energy, the other is equivalent to the temperature over $1000 \mathrm{~K}$.

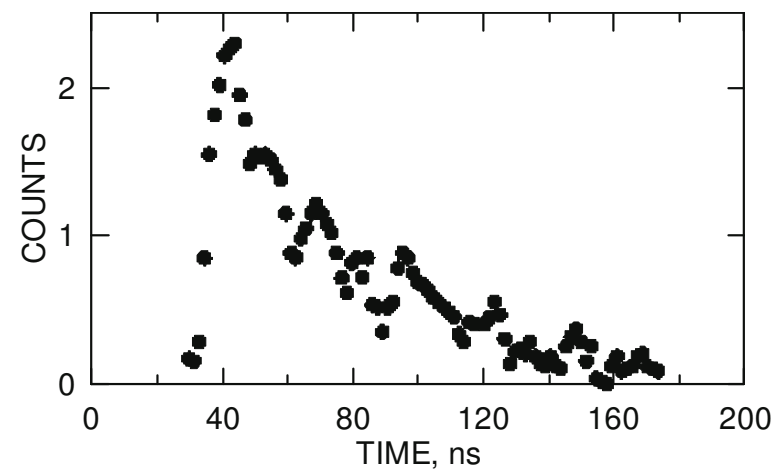

Fig. 71. Time of flight spectrum of o-Ps escaping from the pores of silica,

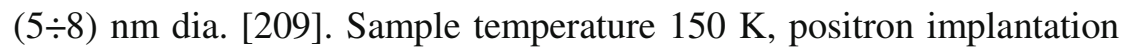
energy $7 \mathrm{keV}$. Ps energy distribution contains two components, corresponding to temperatures $(145 \pm 10) \mathrm{K}$ and $(1260 \pm 15) \mathrm{K}$.

Positronium precursor, qPs (see Sec.4) is formed in the bulk and in its diffusive motion can find itself near a void surface and be emitted into small intermolecular free volume or into the pore. In silica or glassy 
media the intermolecular voids are abundant, the o-Ps lifetime observed in them, $\tau_{2}$, is of the order of $1.5 \mathrm{~ns}$. The probability of Ps location in a pore depends on specific pore surface area (total pore surface per mass unit) $S$. The calculations of the fraction $\kappa$ of o-Ps annihilating in pores were first performed by Brandt and Paulin [210] and modified later by Venkateswaran et al. [211]:

$$
\kappa=\frac{I_{4}}{I_{3}+I_{4}}=\frac{3}{2} \beta\left[1-\beta^{2}+(1+\beta)^{2} e^{(-2 / \beta)}\right]
$$

where $\beta$ is a dimensionless parameter proportional to pore surface $S: \beta=S \rho\left(D \tau_{3}\right)^{1 / 2} / 3, \rho-$ the bulk density, $D$ - the Ps diffusion coefficient; $\tau_{3}$ is the o-Ps lifetime in the bulk material. An example of the fraction $\kappa$ of Ps annihilating in the pores at various specific pore surfaces in Vycor glass is shown in Fig. 72 [212]; the surfaces are from 12.5 to $350 \mathrm{~m}^{2} / \mathrm{g}$. The Ps diffusion coefficient found in the experiments of this kind is $(2 \div 5) 10^{-5} \mathrm{~cm}^{2} / \mathrm{s}$.

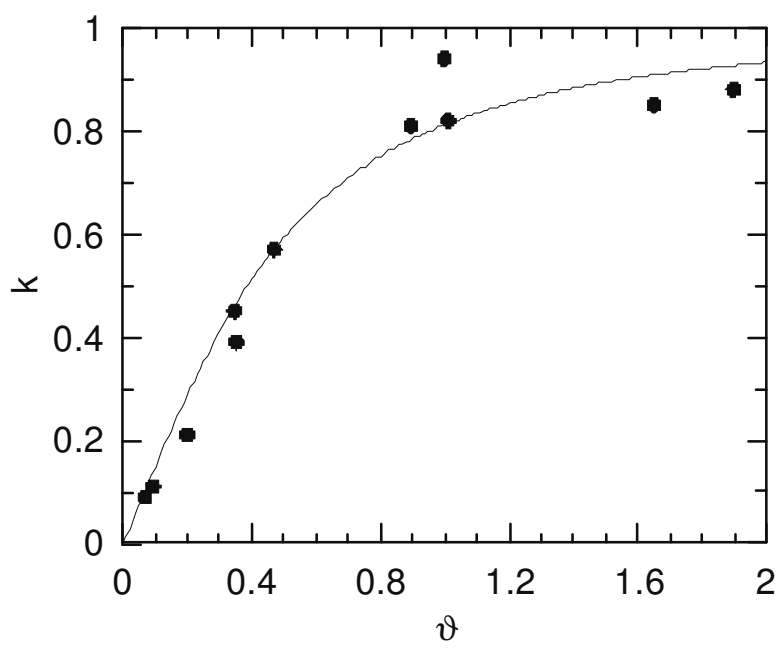

Fig. 72. Fraction $\kappa$ of ortho-po-sitronium annihilating in the pores as a function of specific pore surface area in Vycor glass [212].

\subsection{Ordered porous structures}

In porous media the pore radii have a rather broad distribution. However, there are structures in which the radii are uniform; all cylindrical channels are of the same diameter. To this class of media belong ordered silicas MCM-41, SBA-15, FSM16 and analogous ones 
[213]. The MCM-41 is synthesized using the long cylindrical micelles of a surfactant, which, on addition of a silica source, reorganize themselves into a bundle of parallel channels with hexagonal symmetry. Typical surfactants are alkyltrimethylammonium bromides; the silica source is tetraetoxysilane (TEOS). Exceptionally large pore radii, up to $6 \mathrm{~nm}$ can be obtained when the surfactant is supplemented by trimethylbenzene [214]. At basic $\mathrm{pH}$ values the condensation of silica occurs around previously formed organic micelles (template) giving the structure as shown in Fig. 73.
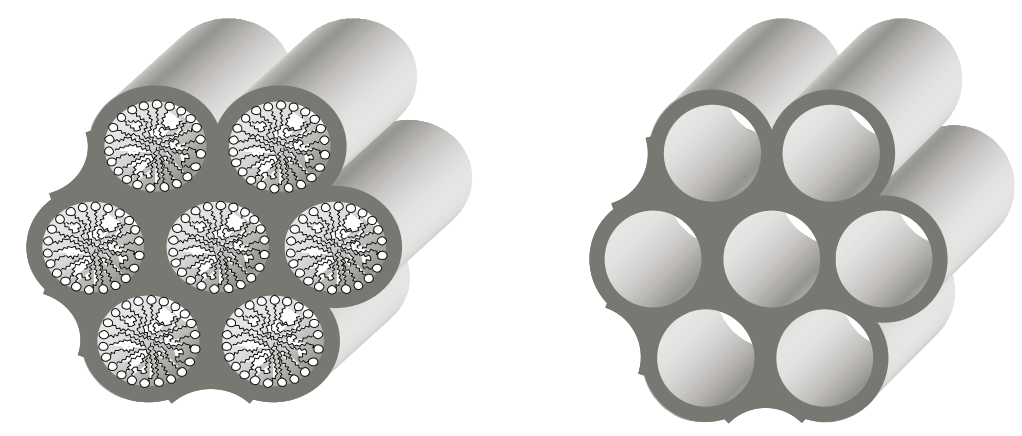

Fig. 73. Structure of MCM-41 ordered silica (drawing by R. Zaleski). left - with micella template; right - after removal of the template.

Removal of the surfactant by calcination yields the final hexagonal pore structure. The pore diameter is easily adjustab-le by changing the alkyl chain length and composition of the surfactant. The PALS method allows to observe the structural chan-ges of the raw material during the process of template removal. The formation of a porous structure in MCM-41 with octa-decyltrimethylammonium tem-plate can be observed in PALS spectra measured as a function of annealing tempe-rature [215].

The spectra shown in Fig.74 were decomposed into 6 exponentials. Up to $400 \mathrm{~K}$ the dominant component was the $3.5 \mathrm{~ns}$ one, resembling that in liquid alkanes and ascribed to the positronium annihilation in the template. There is also a very long-lived component $(100 \div 110)$ ns belonging to Ps annihilation in the spaces between the grains. Very characteristic is the stepwise appearance of a new component at (400-420) K whose lifetime rises with temperature from about $20 \mathrm{~ns}$ to $43 \mathrm{~ns}$ at $550 \mathrm{~K}$ (diamonds in Fig. 74). This final lifetime corresponds to the empty long cylinder of radius $1.52 \mathrm{~nm}$, it means all template is already removed. The diameter of the evacuated volume is constant and thus the rise of lifetime describes the fragmentation of micelles and the expansion of gaps between the fragments. 


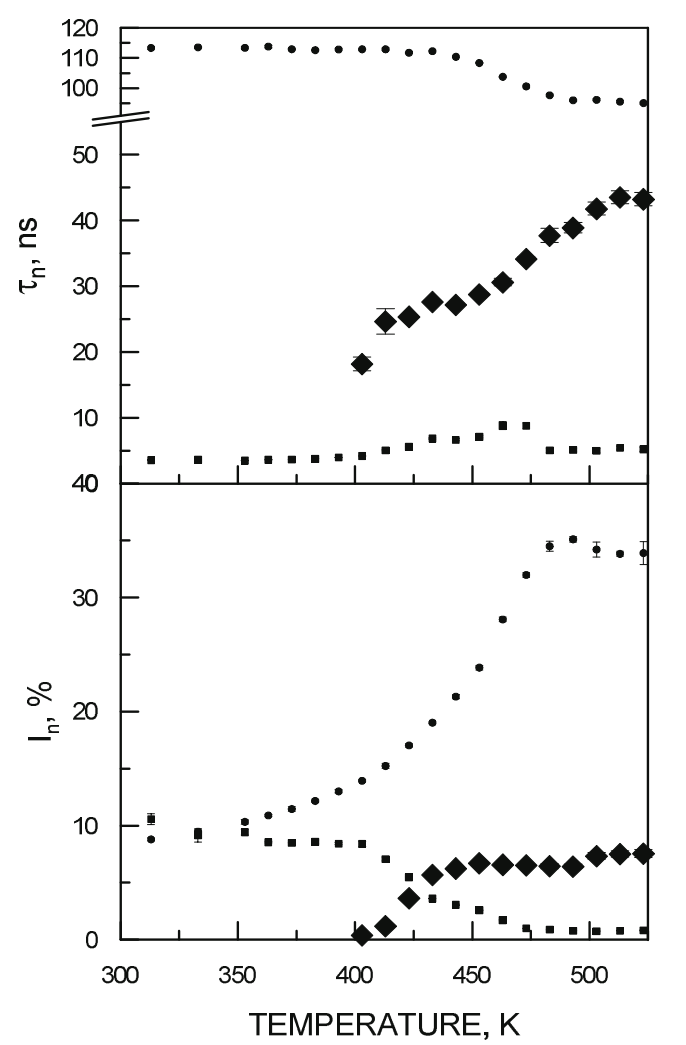

Fig.74. Temperature dependence of three longest o-Ps lifetimes in raw MCM-41. Template removal. From [215] and R. Zaleski, Ph. D. Thesis.

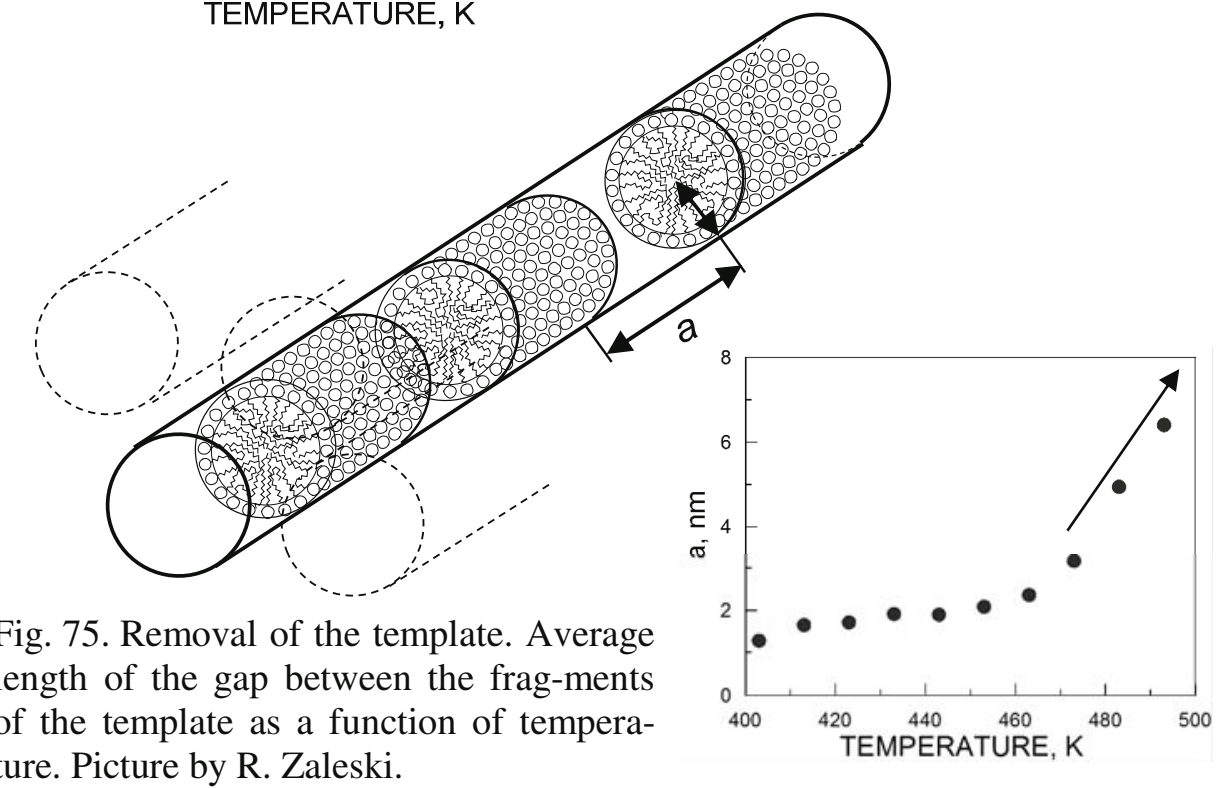

Using the ETE model for cylinders of finite length one can calculate the length of these empty gaps, rising gradually with temperature as shown in Fig.75. The formation of pores is accompanied by the growth of 
$110 \mathrm{~ns}$ component which is produced by o-Ps atoms escaping from the pores to surrounding intergrain vacuum due to short pore length (small sizes of MCM grains).

In the calculations of the o-Ps lifetime in large pores, described above, the processes of pick-off and intrinsic decay only are accounted. Already at $R=2.3 \mathrm{~nm}$ the pick-off probability becomes equal to the intrinsic one. For larger voids pick-off becomes a second-rank process, and other effects neglected at small radii can come to prominence.

\section{SURFACES, FILMS, MEMBRANES, COATINGS}

Describing the positron annihilation at pore formation in MCM-41 we neglected the interaction of o-Ps with surfaces. In some cases this can be an important factor changing the lifetime characteristics, in particular when the pore surface is coated with a conducting material. For example, after template calcination, i.e. burning out the cylindrical micellae, a certain amount of carbon is deposited in the form of spots on the walls of the silica skeleton, giving the sample a characteristic brownish hue. Liquid nitrogen adsorption indicates that the specific surface area can exceed $1000 \mathrm{~m}^{2} / \mathrm{g}$, at the same time the o-Ps lifetime spectrum for such a sample shows a weak and rather short-lived component. In the case of cetylpyridinum chloride template the long-lived component was found to be about $3.5 \mathrm{~ns}$ only [216]. The carbon deposits can be removed by oxygen flow through the sample at $\approx 750 \mathrm{~K}$. With prolonged oxygen treatment the content of carbon decreases, but pore area and volume experience only a slight increase, i.e. the porosity parameters do not change too much. However, in the PALS measurements one observes radical changes of the lifetime; in well purified samples the long-lived component is close to $110 \mathrm{~ns}$ [216]. The carbon on the walls not only quenches positronium, but also reduces its formation probability (Fig.76).

Conventional positron sources (radioactive materials) are not suitable to the study of thin layers, due to continuous energy spectrum of positrons, extending from 0 to $(0.5 \div 1.0) \mathrm{MeV}$, and thus giving a broad distribution of their ranges, from the surface to almost $1 \mathrm{~mm}$. To study thin foils one has to stack them to get a total sample thickness of the order of $1 \mathrm{~mm}$. If there is a layer of material under study on a substrate, the measurement is still possible provided the thickness of layer is over 20 $\mu \mathrm{m}$ thick and the lifetimes are much longer than those in the substrate (or the substrate is entirely free of positronium). 


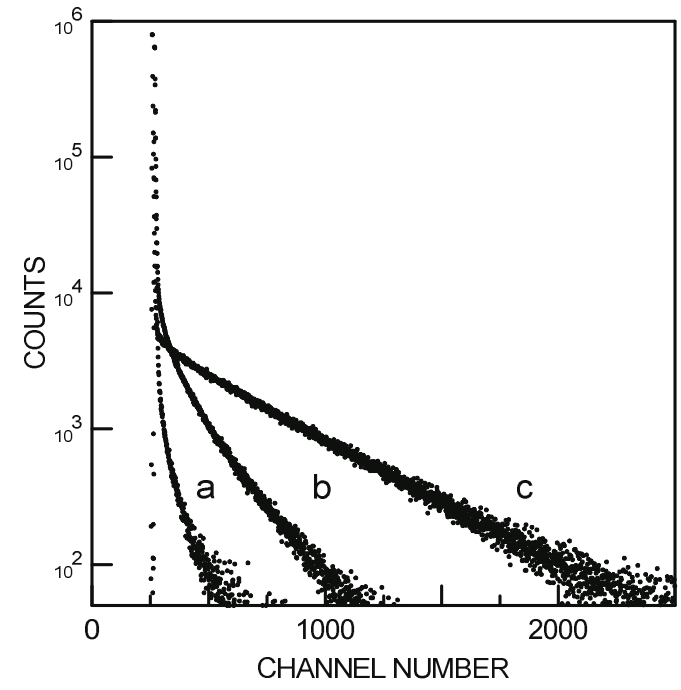

Fig. 76. The PALS spectra of MCM-41 [216]: a - directly after template pyrolysis, $\mathrm{b}-$ after $6 \mathrm{~h}$ oxygen treatment, $\mathrm{c}-$ after $10 \mathrm{~h}$ (and more).

The observed long-lived component is then of low intensity, but the lifetime is easy to determine - all positrons penetrating the nonporous substrate decay rapidly, without disturbing the long-lived part. Such measurements were performed e.g. by Itoh et al. [217] with porous silicon where a quite intense component with the lifetime of $25 \mathrm{~ns}$ was observed. The porous structure of silicon depends strongly on the method of preparation, thus a large scatter of data is observed, for example Shantarovich et al. [218] observed the lifetime of $7.1 \mathrm{~ns}$; while Dannefaer et al. [219] - up to $90 \mathrm{~ns}$.

The introduction of slow positron beams opened the way to positron annihilation studies of defect profiles. Controlling the positron energy $E$ one can choose the mean depth at which the positron annihilates. The average positron range is:

$$
\bar{z}=A E^{n}
$$

where the constant $A$ was found empirically to be $A=400 / \rho$ in angstroms for energy $E$ in $\mathrm{keV}$ and density $\rho$ in $\mathrm{g} / \mathrm{cm}^{3} ; n=1.6$. The stopping profile of monoenergetic positrons is:

$$
P(z)=\frac{m z^{m-1}}{z_{0}^{m}} \exp \left[-\left(z / z_{0}\right)^{m}\right]
$$

where $m=1.9$ and $z_{0}$ is related to the average range $\bar{z}$ by the equation:

$$
\bar{z}=z_{0} \Gamma(1+1 / m)
$$


( $\Gamma$ is the Euler gamma function, $\Gamma(1+1 / \mathrm{m})$ is about 0.85$)$. The distribution of ranges for several positron energies is shown in Fig. 77.

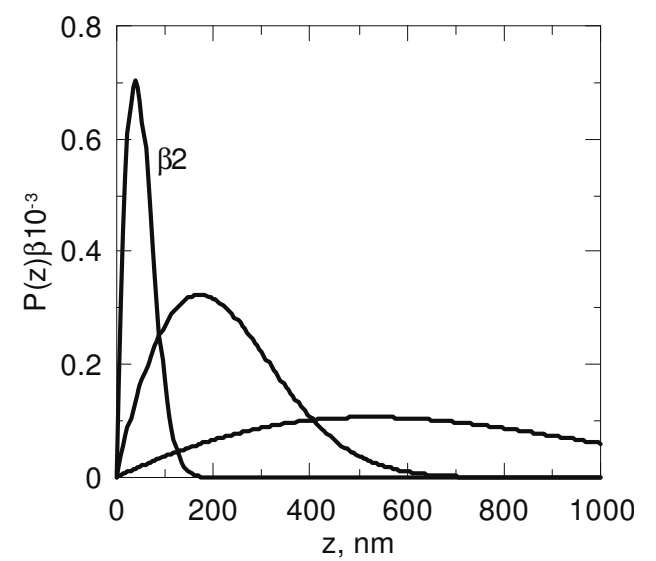

Fig. 77. Distribution of positron ranges in silicon for monoenergetic positrons of energy 2,5 and $10 \mathrm{keV}$.

The simplest way to observe the defects in thin layers using a slowpositron beam technique is to apply the DBARL method, giving the values of $S$ and $W$ parameters ${ }^{7}$.

Monoenergetic positron beams are particularly useful in the studies of membranes. An industrial membrane is often composed of several layers. For example, the membrane separating water and isopropanol contains three layers of polyamide skin, a modified polyacrylonitrile porous membrane and a support. A determination of pore size and distribution is possible when the positron range is fitted to the thickness of layers [220].

A similar situation appears in the case of coatings, e.g. Cao et al. [221] have studied the degradation of aircraft coating due to UV exposition. The coating consisted of polyurethane topcoat, epoxy primes and the aluminium surface treatment layer optimizing primer adhesion. It was found that UV irradiation decreases the $S$ parameter in all layers, that means a reduction of void sizes or of their concentration. Authors propose to explain it as the result of increasing the number of cross-links in the epoxy layer.

Positron methods allow to distinguish open and closed pores. For example, to reduce the RC time constant in very fast integrated circuits, the insulating layers should have possibly low dielectric constant. One can substitute the bulk insulator by the same but porous. One of such

\footnotetext{
${ }^{7}$ The technique of variable energy positron beam is very often used in the version with positrons (not positronium), trapped in structural defects of conducting materials [VII].
} 
insulators developed by IBM is methyl-silsesquioxane (MSSQ) with addition of porogen. As a porogen the $\operatorname{poly}(\varepsilon$-caprolactane) based polymer (PCL) is used. During thermal treatment of such films a decomposition of the porogen and volatilization of the resulting fragments occurs, leaving closed nanopores [222], whose sizes can be determined by the PALS method. With increasing porogen content the isolated individual voids connect, forming a channel open to the film surface. Ortho-positronium, which was trapped in voids, now can migrate along such a channel and escape into vacuum. When outside of the film, its lifetime is $\approx 142 \mathrm{~ns}$, so the appearance of such a lifetime is an indication of pore interconnectivity. The o-Ps in open vacuum annihilates via threequantum emission, thus the intensity of such decays can be a measure of the escape probability.

Considering the relation between o-Ps lifetime and the pore size one should take into account the adsorption of residual gases, particularly at very low temperatures. Adsorption and then desorption at increased temperature can produce a non-monotonic shape of $\tau v s$. T dependence. A drastic example of such effect was demonstrated e.g. in the paper by Uedono et al.[223]. The effect of lifetime changes due to surface covering can appear also in the case of grafted pores.

\section{SUMMARY}

Positronium studies represent a fragment of more broad positron spectroscopy, operating the neutral particle of small mass, thus being a non-destructive probe of the matter which it penetrates. Location of Ps in the regions of negligible electron density (vacancies, intermolecular spaces, pores) gives the possibility of an insight into subnanometric irregularities of the structure. The most useful is positronium in two cases:

- in polymers the "free volumes" are important for macroscopic physical properties of these media. Positronium allows to determine the sizes of individual voids, their distribution and concentration,

- in porous media positronium probes not only open, but also closed pores. The investigations can be performed at arbitrary temperature. 
Positronium techniques are now at the stage of practical application, however, they still need to improve the models describing the relation between annihilation processes and the structure of matter. The study of fundamental properties, e.g. of the behaviour of confined particle are also important.

\section{ACKNOWLEDGEMENTS}

Author wishes to thank Gerhard Brauer for helpful discussion and comments; Tetsuya Hirade, Reinhard Krause-Rehberg, Frans H.J. Maurer, Wojciech Osoba, Vladimir Slugeň, Radek Zaleski for supplying some illustrative materials, Bozena Zgardzińska for preparation of pictures and making-up the final graphic version of this paper.

\section{REFERENCES}

[1] M. Deutsch, Phys. Rev. 82, 455 (1951).

[2] G.S. Adkins, E.D. Pfahl, Phys. Rev. A58, 3552 (1998).

[3] J. Govaerts, M. Van Caillie, Phys. Lett. B 381, 451 (1996);

A. Czarnecki, S.G. Karshenboim, Proc. 14th Intl. Workshop on High Energy Physics and Quantum Field Theory, May 1999, Moscow.

[4] R.E. Bell and R.L. Graham, Phys. Rev. 90, 644 (1953).

[5] A.T.G. Ferguson and G.M. Lewis, Phil. Mag. 44, 1339 (1953).

[6] J.S. Nico, D.W.Gidley, A. Rich, P.W. Zitzewitz, Phys. Rev. Lett. 65, 1344 (1990).

[7] G.S. Adkins, R.N. Fell, J. Sapirstein, Phys. Rev. Lett. 84, 5086 (2000).

[8] R.S. Vallery, P.W. Zitzewitz, D.W. Gidley, Phys. Rev. Lett. 90, 03402 (2003).

[9] J. Facetti, J.C. Abbé, G. Duplâtre, Nucl. Instrum. Methods 174, 317 (1980).

[10] A. Latuszyński, Yu.V. Yushkevich, V.A. Bystrov, R. Misiak, J. Wawryszczuk, T. Goworek, Acta Phys. Polon. A83, 345 (1993).

[11] J. R. Poulsen, M. Eldrup, J. Lettry, Nucl. Instrum. Methods Phys. Res. 95, 260 (1995).

[12] T. Hirade, H. Toyokawa, T. Ohdaira, R. Suzuki, H. Ohgaki, Material Sci. Forum 445-446, 474 (2004). 
[13] D.G. Costello, D.E. Groce, D.F. Herring, J.W. McGowan, Phys. Rev. B5, 1433 (1972).

[14] K.F. Canter, P.G. Coleman, T.C. Griffith, G.R. Heyland, J. Phys. B5, L167 (1972).

[15] B. Jaduszliwer, W.C. Keever, D.A.L. Paul, Can. J. Phys. 50, 1414 (1972).

[16] A.P. Mills Jr. and E.M. Gullikson, Appl. Phys. Lett. 49, 1121 (1986).

[17] A.P. Mills Jr., Phys. Rev. Lett. 41, 1828 (1978).

[18] G. Consolati, R. Ferragut, A. Galarneau, F. Di Renzo, F. Quasso, Chem. Soc. Rev. 42, 3821 (2013).

[19] A.P. Mills Jr., Phys. Rev. Lett. 46, 717 (1981).

[20] K. Michishio, T. Tachibana, R.H. Suzuki, K. Wada, A. Yagishita, T. Hyodo, Y. Nagashima, Appl. Phys. Lett 100, 254102 (2012).

[21] T. Goworek, W. Górniak, J. Wawryszczuk, Nucl. Instrum. Methods Phys. Res. A331, 560 (1992).

[22] M. Laval, M. Moszyński, R. Allemand, E. Comoreche, P. Guinet, R. Odru, J. Vacher, Nucl. Instrum. Methods Phys. Res. 206, 169 (1983).

[23] F. Becváŕ, J. Cížek, I. Procházka, I. Janotová, Nucl. Instrum. Methods Phys. Res., Sect. A 539, 372 (2005).

[24] K. Maier, R. Myllylä, in "Positron Annihilation".Eds. R.R. Hashiguci, K. Fujiwara, Japan Inst. of Metals, Sendai 1982. p.829; H.E. Schaefer, W. Weiler, in "Positron Annihilation " Eds. P.C. Jain , E.M. Singru, K. Gopinathan, World Sci, 1985, p.584.

[25] Y. Shirai, M. Sakamura, I. Shishido, M. Yamaguchi, J. Japan Inst. Metals 59, 679 (1995).

[26] P Chalermkarnon, I. Shishido, M. Yuga, H. Araki, Y. Shirai, J. Japan Inst. Metals 66, 1004 (2002).

[27] K.G. Lynn, W.E. Frieze, P.J. Schultz, Phys. Rev. Lett. 52, 1137 (1984).

[28] F. Reurings, A. Laakso, K. Rystölä, A, Pelli, K. Saarinen, Appl. Phys. Sci. 252, 3154 (2006).

[29] M. Butterling, W. Anwand, G. Brauer, T.E. Cowan, A. Hartmann, M. Jungmann, K. Kosev, R. Krause-Rehberg, A. Krille, R. Schewengner, phys. stat. sol. (a) 207, 334 (2010).

[30] R.L. Garwin, Phys. Rev. 91, 1571 (1953).

[31] A. Dupasquier, P. De Natale, A. Rolando, Phys. Rev. B43, 10036 (1991). 
[32] O.E. Mogensen, G. Kvajić, M. Eldrup and M. Milošević-Kvajić, Phys. Rev. 84,71 (1971).

[33] W. Brandt, G. Coussot, R. Paulin, Phys. Rev. Lett. 23, 522 (1969); also G. Coussot, Ph.D. Thesis, Orsay 1970.

[34] A. Greenberger, A.P. Mills, A. Thompson, S. Berko, Phys. Lett. A32, 72 (1970).

[35] P. Kirkegaard, M. Eldrup, Computer Phys. Comm. 3, 240 (1972);

P. Kirkegaard, N.J. Pedersen, M. Eldrup, Ris $\varnothing$ Report M2740, Ris $\emptyset$ National Laboratory, Denmark 1989.

[36] J. Kansy, Nucl. Instr.\& Meth. A374, 235 (1996).

[37] A. Shukla, M. Peter and L. Hofmann, Nucl. Instr. \& Meth. A335, 310 (1993).

[38] T. Suzuki, N. Oshima, E. Hamada, T. Ogawa, M. Murakami, Y. Ito, Material Sci. Forum 255-257, 308 (1997).

[39] W.F. Magalhães, J.C. Abbé and G. Duplâtre, Struct. Chem. 2, 399 (1991).

[40] T. Goworek, C. Rybka, Acta Phys. Polon. A50, 121 (1976).

[41] H.O. Anger, IEEE Trans. Nucl. Sci. 13, 380 (1966).

[42] A.P. Jeavons, IEEE Trans. Nucl. Sci. 23, 640 (1976).

[43] P.E. Buson, P. Descouts, A. Dupanloup, A.A. Manuel, E.Perrcard, M. Peter, R. Sachot, Helv. Phys. Acta 55, 100 (1982).

[44] T.E. Jackman, P.C. Lichtenberger and C.W. Shulte, Appl. Phys. 5, 259 (1975).

[45] L. Liszkay, C. Corbel, L. Baroux, P. Hautojärvi, M. Bayham, A.W. Brinkman, S. Tatarenko, Appl. Phys. Lett. 64, 1380 (1994).

[46] K.F. Ho, C.D. Beling, S. Fung, V.K.W. Cheng, M.K. Ng, A.M. Yip, Rev. Sci. Instrum.74, 4779 (2003).

[47] W. Gustaw, K. Zaleski, Proceedings of the Conference, "Physics for Industry", Cracow 1980, p.156-157 ; in Polish.

[48] K.G. Lynn, J.R. MacDonald, R.A. Boie, L.C. Feldman, J.D. Gable, M.F. Robbins, E. Bonderup, J. Golovchenko, Phys. Rev. Lett. 38, 241 (1977); details: P.Asoka-Kumar, M. Alatalo, V.J. Gosh, A.C. Kruseman, B. Nielsen, K.G. Lynn, Phys. Rev. Lett. 77, 2097 (1996).

[49] J.D. McGervey, V.F. Walters, Phys. Rev. B2, 2421 (1970).

[50] I.K. MacKenzie, B.T.A. McKee, Appl. Phys. 10, 245 (1976).

[51] Y. Kishimoto, S. Tanigawa, in: "Positron Annihilation" Eds. P.G. Coleman, S.C. Sharma, L.M. Diana, North Holland, Amsterdam 1982 , p. 815. 
[52] A. Ore, Univ. i Bergen Arbok 1949, Naturvitenskapelig rekke Nr.9, Bergen, 1949.

[53] O.E. Mogensen, J. Chem. Phys. 60, 998 (1974).

[54] V.M. Byakov, V.I. Goldanskii and V.P. Shantarovich, Dokl. Akad. Nauk SSSR 219, 633 (1974).

[55] S.J. Tao, Appl. Phys. 10, 67 (1976).

[56] Y.Ito, J. Radioanal. Nucl. Chem. 210, 327 (1996).

[57] S.V. Stepanov, C.L. Wang, Y. Kobayashi, V.M. Byakov, K. Hirata, Radiat. Phys. Chem. 58, 403 (2000).

[58] S.V. Stepanov, V.M. Byakov, T. Hirade , Radiat. Phys. Chem. 76, 90 (2007); also: S.V. Stepanov, V.M. Byakov, D.S. Zvezhinski, G. Duplatre, R.R. Nurmukhamedov, P.S. Stepanov, Adv. Phys. Chem., Vol.2012, art. ID 431962.

[59] A. Garcia, S.M. Pimblott, H. Schut, A. van Veen, J. Phys. Chem. B106, 1124 (2002).

[60] J.M. Wiesenfeld, E.P. Ippen, Chem. Phys. Lett. 73, 47 (1980).

[61] A.P. Mills jr., W.S, Crane, Phys. Rev. A 31, 593 (1985).

[62] Y. Nagashima, Y. Morinaka, T. Kurihara, Y. Nagai, T. Hyodo, T. Shidara, K. Nakahara, Phys. Rev. B 58, 12676 (1998).

[63] M. Eldrup, A. Vehanen, P.J. Schultz, K.G. Lynn, Phys. Rev. B32, 7048 (1985).

[64] T. Mukherjee, D. Gangopadhyay, S.K. Das, B.N. Ganguly, B. Dutta-Ray, J. Chem. Phys. 110, 6844 (1999).

[65] T. Mukherjee, S.K. Das, B.N. Ganguly, B. Dutta-Ray, Phys. Rev. B57, 13363 (1998).

[66] D. Gangopadhyay, B.N. Ganguly, T. Mukherhee, B. Dutta-Roy, J. Phys. Condens. Matter 11, 1463 (1999).

[67] A. Sommerfeld, H. Welker, Ann. d. Phys. 32, 56 (1938).

[68] G.M. Bartenev, A.D. Tsyganov, E.P. Prokop'ev, A.Z. Varisov, Fizika 7, 71 (1970) [Soviet Physics Journal 13, 893 (1970)].

[69] B. Zgardzińska, T. Goworek, Chem. Phys. 421, 10 (2013).

[70] H. Nakanishi, Y,C. Jean, in: "Positron and Positronium Chemistry” (Eds. D.M. Schrader, Y.C. Jean.), Elsevier, 1988.

[71] S.J. Tao, J. Chem. Phys. 56, 5499(1972).

[72] M. Eldrup, N.J. Pedersen, J.N. Sherwood, Phys. Rev. Lett. 43, 1407 (1979).

[73] D. Lightbody, J.N. Sherwood, M. Eldrup, Chem. Phys. Lett. 70, 487 (1980).

[74] M. Eldrup, D. Lightbody, J.N. Sherwood, Chem. Phys. 63, 51 (1981). 
[75] D. Lightbody, J.N. Sherwood, M. Eldrup, Mol. Cryst. Liq, Cryst. 96, 197 (1983).

[76] D. Lightbody, J.N. Sherwood, M. Eldrup, Chem. Phys. 93, 475 (1985).

[77] M. Eldrup, D. Lightbody, J.N. Sherwood, Faraday Discuss. 69, 175 (1980).

[78] H. Nakanishi, Y. Ujihira, J. Chem. Phys. 86, 4446 (1982).

[79] B. Zgardzińska, Acta Phys. Polon. 125,700 (2014).

[80] R. Lenc, Thesis (Silesian University, Katowice, 1988)

[81] B. Jasińska, A.E. Kozioł, T. Goworek, Radioanal. Nucl. Chem. 210, 617 (1996).

[82] L. Larrimore, R.N. McFarland, P.A. Sterne, A.L.R. Bug, J. Chem. Phys. 113, 10642 (2000).

[83] P.A. Sterne, L. Larrimore, P. Hastings, A.L.R. Bug, Radiat. Phys. Chem. 68, 409 (2003).

[84] M. Deutsch, S.C. Brown, Phys. Rev. 85, 1047 (1952).

[85] A. Bisi, A. Fiorentini, E. Gatti, L. Zappa, Phys. Rev. 128, 2195 (1962).

[86] T. Goworek, A. Badia, G. Duplâtre, J. Chem. Soc. Faraday Trans. 90, 1501 (1994).

[87] A.P. Mills, Jr., J. Chem. Phys. 62, 2646 (1975).

[88] G. Duplâtre, Z. Kajcsos, T. Goworek, L. Varga, L. Liszkay, I. Billard, K. Lázár, J. Radioanal. Nucl. Chem. 211, 225 (1996).

[89] S. V. Stepanov, V.M. Byakov, J. Chem. Phys. 116, 6178 (2002); S.V. Stepanov, V.M. Byakov, Y. Kobayashi, Phys. Rev. B72, 054205 (2005).

[90] W. Brandt, J. Wilkenfeld, Phys. Rev. B12, 2579 (1975).

[91] A. Bisi, F. Bisi, A. Fasana, L. Zappa, Phys. Rev. 122, 1709 (1961).

[92] W. Górniak, T. Goworek, C. Rybka, Chem. Phys. Lett. 187, 537 (1991).

[93] A. Bisi, G. Gambarini, L. Zappa, Nuovo Cimento 2D, 1465, (1983).

[94] P.C. Jain, M. Eldrup, J.N. Sherwood, in: „Positron Annihilation”, Eds. P.G. Coleman, S.C. Sharma, L.M. Diana, North Holland, 1982, p.674.

[95] T. Goworek, C. Rybka, phys. stat. sol.(b) 81, 565 (1977).

[96] T. Goworek, C. Rybka, J. Wawryszczuk, phys. stat. sol.(b) 84, K49 (1978).

[97] T. Goworek, C. Rybka, J. Wawryszczuk, phys. stat. sol. (b) 89, 253(1978). 
[98] M. Eldrup, O.E. Mogensen, J. Bilgram, J. Glaciology, 21, 101 (1978).

[99] G. J. Small, J. Chem. Phys. 52, 656 (1970).

[100] R. de Zafra, W. Joyner, Phys. Rev. 112, 19 (1958).

[101] H.S. Landes, S. Berko, A.J. Zuchelli, Phys. Rev. 103, 828 (1956).

[102] T. Goworek, C. Rybka, R. Wasiewicz, J. Wawryszczuk, phys. stat. sol.(b) 113, K9 (1982).

[103] C.P. Brock, J.D. Dunitz, Acta Crystallogr. B38, 2218 (1982).

[104] T. Goworek, C. Rybka, J. Wawryszczuk, R. Wasiewicz, Chem. Phys. Lett. 106, 482 (1984).

[105] T. Goworek, C. Rybka, J. Radioanal. Nucl. Chem. 242, 225 (1999).

[106] T. Goworek, T. Suzuki, E. Hamada, K. Kondo, Y. Ito, Chem. Phys. 255, 347 (2000).

[107] T. Goworek, J. Wawryszczuk, R. Zaleski, Chem. Phys. Lett. 387, 433 (2004).

[108] A. Craievich, J. Doucet, I. Denicolò, Phys. Rev. B32, 4164 (1985).

[109] T. Goworek, R. Zaleski, J. Wawryszczuk, Chem. Phys. 295, 243 (2003).

[110] T. Asano, J. Phys. Soc. Jpn 54, 1403 (1985).

[111] T. Goworek, M. Pietrow, R. Zaleski, B. Zgardzińska, Chem. Phys. 355, 123 (2009); also T. Goworek, B. Zgardzińska, M. Pietrow,

J. Wawryszczuk, Material Sci. Forum 733, 67 (2013).

[112] B. Zaslow, M.E. Zandler, Am. J. Phys. 35, 118 (1967)

[113] G. Bastard, E. E. Mendez, L.L. Cheng, L. Esaki, Phys. Rev. B26, 1974 (1982).

[114] J. Bruce, J.N. Sherwood, N.J. Pedersen, M. Eldrup, in: „Positron Annihilation", Eds. P.C. Jain, R.M. Singru, K.P. Gopinathan, World Sci. Publ. Co. Singapore, 1985, p.181.

[115] D.N. Bolshutkin, V.M. Gasan, A.I. Prokhvatilov, Zh. Strukt. Khim. 12, 734 (1971), in Russian.

[116] S.J. Wang, Y.C. Jean, in: "Positron Annihilation", Eds. L. Dorikens-Vanpraet, M. Dorikens, D. Segers, World Sci. Publ. Co, Singapore 1988, p.809.

[117] E. Gregoryanz, M.J. Clouter, N.H. Rich, R. Goulding, Phys. Rev. B58, 2497 (1998).

[118] J. Doucet, I. Denicolo, A.F. Craievich, J. Chem. Phys. 75, 1523 (1981).

[119] T. Goworek, J. Wawryszczuk, R. Zaleski, B. Zgardzińska, Radiat. Phys. Chem. 76, 185 (2007). 
[120] E.B. Sirota, D.M. Singer, J. Chem. Phys. 101, 10873 (1994).

[121] J.D. Hoffman, C.P. Smyth, J. Am. Chem. Soc. 71, 431 (1949).

[122] R. Lenc, Chem. Phys. 131, 443 (1989).

[123] Y. Kim, L. Strauss R.G. Snyder, J. Phys. Chem. 93, 7520 (1989).

[124] J.F. Kincaid, H. Eyring, J. Chem. Phys. 6, 620 (1938). 71.

[125] Y. Ujihira, T. Ryuo, Y. Kobayashi, T. Nomizu, Appl. Phys. 16, 71 (1978).

[126] D.A.L. Paul, R.L. Graham, Phys. Rev. 106, 16 (1957).

[127] J. Wackerle, R. Stump, Phys. Rev. 106, 18 (1957).

[128] R.A. Ferrell, Phys. Rev. 108, 167 (1957).

[129] L.O. Roelig, in: Positron Annihilation, Eds. A.T. Stewart, L.O. Roelig, Academic Press, New York 1967, p.127.

[130] A.P. Buchikhin, V.I. Goldanski, V.P. Shantarovich, Pis'ma Zh. Exp. Teor. Fiz.12, 624 (1971), in Russian.

[131] O.E. Mogensen, F.M. Jacobsen, Chem. Phys. 73, 223 (1982).

[132] R.C. Tolman, J. Chem. Phys.17, 333 (1949).

[133] W.S. Ahn, M.S. Jhon, H. Pak, S. Chang, J. Colloid Interface Sci. 38, 605 (1972).

[134] V.M. Fenelonov, G. G. Kodenyov, V.G. Kostrovsky, J. Phys. Chem. 105, 1050 (2001).

[135] L.I. Schiff, Quantum Mechanics, McGraw-Hill, New York 1977, Chapter 2.

[136] B. Zgardzińska, T. Goworek, Chem. Phys. 405, 32 (2012).

[137] B. Zgardzińska, T. Goworek, Chem. Phys. 411, 1 (2013).

[138] R.B. Gregory, K.J. Chai, Material Sci. Forum 105-110, 1573 (1992).

[139] K. Kotera, T. Saito, T. Yamanaka, Phys. Lett. A345, 184 (2005).

[140] S.V. Stepanov, V.M. Byakov, G. Duplâtre, D.S. Zvezhinskiy, Y.V. Lomachuk, phys. stat. sol. C6, 2476 (2009).

[141] S.V. Stepanov, D.S. Zvezhinski, G. Duplâtre, V.M. Byakov, Yu.Yu. Batskikh, P.S. Stepanov, Material Sci. Forum 666, 109 (2011).

[142] F.M. Jacobsen, Ris $\varnothing$ Report R-433, Ris $\varnothing$, 1981; F.M. Jacobsen, O.E. Mogensen, G. Trumpy, Chem. Phys. 69, 71 (1982).

[143] F.M. Jacobsen, M. Eldrup, O.E. Mogensen, Chem. Phys. 50, 393 (1980).

[144] B. Zgardzińska, M. Paluch, T. Goworek, Chem. Phys. Lett. 491, 160 (2010).

[145] M. Avrami, J. Chem. Phys. 7, 1103 (1939). 
[146] A. Wypych, V. Guinet, A. Hedoux, Phys. Rev. B76, 144202 (2007)

[147] Ch. Zhao, Y. Ujihira, J. Radioanal. Nucl. Chem. 211, 137 (1996).

[148] B. Jasińska, A.E. Kozioł, T. Goworek, Material Sci. Forum 255257, 308 (1997).

[149] H. Eyring, J. Chem. Phys. 4, 283 (1936).

[150] G. Dlubek, J. Pionteck, Y. Yu, S. Thränert, M. Elsayed, E.

Badawi, R. Krause-Rehberg, Macromol. Chem. Phys. 209, 1920 (2008).

[151] G. Dlubek, J. Wawryszczuk, J. Pionteck, T. Goworek, H. Kaspar, K.H. Lockhaas, Macromolecules 38, 427 (2005).

[152] P. Winberg, M. Eldrup, F.H.J. Maurer, J. Chem. Phys. 136, 244902 (2012).

[153] Q. Deng, Y.C. Jean, Macromolecules, 26, 30 (1996).

[154] R.M. Nieminen, in: "Positron Solid State Physics", Eds. W. Brandt, A. Dupasquier, North Holland 1983, p.385.

[155] Z. Yu, J.D. McGervey, A.H. Jamieson, R. Simha, Macromolecules 28, 6268 (1995).

[156] Y.C. Jean, Macromolecules, 29, 5756 (1996).

[157] A.H. Baugher, W.J. Kossler, K.G. Petzinger, Macromolecules 29, 7280 (1996).

[158] R. Simha, T. Somcynsky, Macromolecules 2, 342 (1969).

[159] G. Dlubek, J. Pionteck, M. Śniegocka, E.M. Hassan, R. KrauseRehberg, J. Polymer Sci Part B 45, 2519 (2007).

[160] Y. Kobayashi, W. Zheng, E.F. Mayer, J. McGervey, A. Jamieson, Macromolecules 22, 2302 (1989).

[161] F.H.J. Maurer, M. Schmidt, Radiat. Phys. Chem. 58, 509 (2000).

[162] K. Okamoto, K. Tanaka, M. Katsube, H. Kita, Y. Ito, Polymer Journal 25, 275 (1993).

[163] W. Ma, A. Andersson, J. He, F.H.J. Maurer, Macromolecules 41, 5307 (2008).

[164] H. Nakanishi, Y.C. Jean, E.G. Smith, T.C. Sandreczki, J. Polym. Sci. Part B 27, 1419 (1989).

[165] J.H. Lind, P.L. Jones, G.W. Pearsall, J. Polym. Sci. Part A 24, 3033 (1986).

[166] J. Serna, J.-Ch. Abbe, G. Duplatre, phys. stat. sol. (a) 115, 389 (1989).

[167] J.J.Singh, A. Eftekhari, B.T. Upchurch, K.S. Burns, NASA Tech. Paper 3034 (1990). 
[168] X. Hong, Y.C. Jean, H. Yang, S.S. Jordan, W.J. Koros, Macromolecules 29, 7859 (1996).

[169] A.Yu.Alentiev, V.P. Shantarovich, T.C. Merkel, V.I. Bondar, B.D. Freeman, Yu.P. Yampolskii, Macromolecules 35, 9513 (2002).

[170] G. Dlubek, J. Pionteck, Y. Yu, S. Thränert, E. Badawi, R. KrauseRehberg, Macromol. Chem. Phys. 209, 1920 (2008).

[171] S. Claes, P. Vanderzande, S. Mullens, M.K. Van Bael, F.H. J. Maurer, Macromolecules 44, 2766 (2011).

[172] K. Ito, Y. Kobayashi, A. Nanasawa, Appl. Phys. Lett. 82, 654 (2003).

[173] H. Borek, W. Osoba, Polymer 42, 2901 (2001).

[174] Y. Ito, Material Sci. Forum 175-178, 308 (1997).

[175] C. Dauwe, Physicalia Mag.12, 211 (1990).

[176] G. Consolati, I. Genco, M. Pegoraro, L. Zanderighi, J. Polym. Sci. Pt.B 34, 357 (1996).

[177] P. Kindl, G. Reiter, phys. stat. sol. (a) 104, 707 (1987) Y. Ito, Polymer 37, 283 (1996).

[178] T. Suzuki, Y. Oki, M. Numajiri, T. Miura. K. Kondo, N. Oshima, Y. Ito, Polymer, 37, 283 (1996).

[179] A. Uedono, T. Kawano, S. Tanigawa, M. Ban, M. Kyoto, T. Uozumi, J. Polym. Sci Part B 34, 2145 (1996).

[180] T. Suzuki, T. Goworek, K. Kondo, E. Hamada and Y. Ito, J. Nucl. Radiochem. Sci. 1, Supplement, 190 (1999); in Japanese.

[181] T. Goworek, R. Zaleski, J. Wawryszczuk, Chem. Phys. 295, 243 (2003).

[182] C.L. Wang, T. Hirade, F.H.J. Maurer, M. Eldrup, N.J. Pedersen, J. Chem. Phys. 108, 4654 (1998).

[183] T. Hirade, F.H.J. Maurer, M. Eldrup, Radiat. Phys. Chem. 58, 465 (2000).

[184] T. Hirade, C.L. Wang, F.H.J. Maurer, M. Eldrup, N.J. Pedersen, Abstract book for The 36th Annual Meeting on Radioisotopes in the Physical Science and Industries, Tokyo, 1998, p.89.

[185] Y. Ito, T. Hirade, E. Hamada, T. Suzuki, Y. Ito, Acta Phys. Polon. A95, 533 (1999).

[186] N. Djourelov, T. Goworek, K. Kondo, T. Suzuki, R. Zaleski, Phys. Lett. A 323, 165 (2004).

[187] B. Zgardzinska, T. Goworek, Acta Phys. Polon. A119, 328 (2011).

[188] V.P. Shantarovich, R.S. Yu, Ya. Kino, Ya. Hama, V.W. Gustov, High Energy Chem. 45, 1 (2011); in Russian. 
[189] F. Nahid, J.D. Zhang, T.F. Yu, C.C. Ling, S. Fung, D.C. Beling, Radiat. Phys. Chem. 60, 529 (2011).

[190] M. Pietrow, J.Wawryszczuk, Material Sci. Forum 666, 89 (2011).

[191] B. Zgardzińska, T. Hirade, T. Goworek, Chem. Phys. Lett. 446, 309 (2007).

[192] M. Welander, F.H.J. Maurer, Material Sci. Forum 105-110, 1811 (1992).

[193] R.A. Naslud, Ph.L. Jones, A. Crowson, Material Sci. Forum 175178, 739 (1995).

[194] G. Dlubek, D. Bamford, O. Henschke, J. Knorr, M.A. Alam, M. Arnold, Th. Lüpke, Polymer, 42, 5381 (2001).

[195] B. Zgardzińska, T. Goworek, Chem. Phys. Lett. 547, 35 (2012).

[196] V.P. Shantarovich, J. Radioanal. Nucl. Chem. 210, 357 (1996).

[197] T. Goworek, K. Ciesielski, B. Jasińska, J. Wawryszczuk, Chem. Phys. Lett. 272, 91 (1997); Mater. Sci. Forum 255-257, 296 (1997).

[198] T. Goworek, K. Ciesielski, B. Jasińska, J. Wawryszczuk, Chem. Phys. 230, 305 (1998).

[199] D.W. Gidley, W.E. Frieze, A.F. Yee, T.L. Dull, H.-M. Ho, E.T. Ryan, Phys. Rev. B60, R5157 (1999).

[200] S. Takada, T. Iwata, K. Kawashima, H. Saito, T. Hyodo, Radiat. Phys. Chem. 58, 781 (2000).

[201] C. Dauwe, B. van Waeyenberge, D. Segers, T. van Hoecke, J. Kuriplach, J. Radioanal. Nucl. Chem. 210, 293 (1996).

[202] T. Chang, M. Xu, X. Zeng, Phys. Lett. A 126, 189 (1987).

[203] T. Goworek, K. Ciesielski, B. Jasińska, J. Wawryszczuk, Radiat. Phys. Chem. 58, 719 (2000).

[204] K.Ciesielski, A.L. Dawidowicz, T. Goworek, B. Jasińska,

J. Wawryszczuk, Chem. Phys. Lett. 289, 41 (1998).

[205] J. Kallmann, D. Enke, S. Thränert, R. Krause-Rehberg, A. Inayar, Colloid Surface 357, 17 (2010).

[206] C. Dauwe, G.Consolati, T. van Hoecke, D.Segers, Nucl. Instr. Meth. A371, 497 (1996).

[207] S.W.H. Eijt, A. van Veen, C.V. Falub, R. Escobar-Galindo, H. Schut, P.E. Mijnarends, F.K. de Theije, A.R. Balkenende, Radiat. Phys. Chem. 68, 357 (2003).

[208] B. Jasińska, A.L. Dawidowicz, T. Goworek, Radiat. Phys. Chem. 58, 723 (2000).

[209] S. Mariazzi, P. Bettotti, R.S. Brusa, Phys. Rev. Lett. 104, 243401 (2010). 
[210] W. Brandt, R. Paulin, Phys. Rev. Lett. 21, 193 (1968).

[211] K. Venkateswaran, K.L. Cheng, Y.C. Jean, J. Phys. Chem. 88, 2465 (1984).

[212] K. Ciesielski, A.L. Dawidowicz, T. Goworek, Acta Phys. Polon. A95, 483 (1999).

[213] C.T. Kresge, M.E. Leonowicz, W.J. Roth, J.C. Vartuli, J.S. Beck, Nature 359, 710 (1992).

[214] A. Galarneau, D. Despantier, R. Dutartre, F. Di Renzo, Microporous Mesoporous Mater. 27, 297 (1999).

[215] R. Zaleski, J. Goworek, A. Borówka, Stud. Surf. Sci. Catal. 160, 471 (2006).

[216] J. Wawryszczuk, J. Goworek, R. Zaleski, T. Goworek, Langmuir 19, 2599 (2003).

[217] Y. Itoh, H. Murakami, A. Kinoshita, Appl. Phys. Lett. 63, 2798 (1993).

[218] V.P. Shantarovich, I.B. Kevdina, Yu.A. Novikov, V.K. Jain, A. Gupta, Fiz. Tv. Tela 38, 2686 (1996).

[219] S. Dannefaer, D. Kerr, D. Craigen, T. Bretagnon, T. Taliercio, A. Foucaran, J. Appl. Phys. 79, 9110 (1996).

[220] Y.C. Jean, H.M. Chen, L.J. Lee, J. Yang, X. Gu, W-S. Hong, K-R Lee, J.Y. Lai, Y-M. Sun, C-C. Hu, Materials Sci Forum 607, 1 (2008).

[221] H. Cao, R. Zhang, J-P Yuan, Y. He, T.C. Sandreczki, Y.C. Jean, Macromolecules 31, 6627 (1998).

[222] C.V. Nguyen, K.R. Carter, C.J. Hawker, J.L. Hedrick, R.I. Jaffe, R.D. Miller, J.F. Remenar, H-W. Rhee, P.M. Rice, M.F. Toney, M. Trollsas, D.Y. Yoon, Chem. Mater. 11, 3080 (1999).

[223] A. Uedono, Z.Q. Chen, R. Suzuki, T. Odahira, T. Mikado, S. Fukui, A. Shiota, S.-I. Kimura, J. Appl. Phys. 90, 2498 (2001). 
Review papers on related subjects:

[I] A. Rich, Rev. Mod. Phys. 53, 127 (1981).

[II] P.J. Schultz and K.G. Lynn, Rev. Mod. Phys. 60, 701 (1988).

[III] T. Goworek, "Proceedings of the $33^{\text {rd }}$ Polish Seminar on Positron Annihilation", Opole 2001, p.141-166, in Polish.

[IV] P. Hautojärvi "Positrons in Solids", Springer, Berlin 1979

[V] R. Krause-Rehberg, H.S. Leipner, „Positron Annihilation in: Semiconductors “, Springer, Berlin 1999.

[VI] A. Dupasquier, A. P. Mills, Jr. "Positron Spectroscopy of Solids", IOS Press, Amsterdam, 1995.

[VII] R.I. Grynszpan, W. Anwand, G. Brauer, P.G. Coleman, Ann. Chim. Sci. Mat. 32, 365 (2007). 